\title{
BLACK HOLE MASS ESTIMATES BASED ON C IV ARE CONSISTENT WITH THOSE BASED ON THE BALMER LINES*
}

\author{
R. J. Assef ${ }^{1,2,13}$, K. D. Denney ${ }^{1,2,14}$, C. S. Kochanek ${ }^{1,4}$, B. M. Peterson ${ }^{1,4}$, S. KozŁowski ${ }^{1}$, N. Ageorges $^{5}$, \\ R. S. Barrows ${ }^{6}$, P. BuschKamp ${ }^{5}$, M. Dietrich ${ }^{1}$, E. Falco ${ }^{7}$, C. Feiz ${ }^{8}$, H. Gemperlein ${ }^{5}$, A. Germeroth $^{8}$, \\ C. J. Grier ${ }^{1}$, R. HofmanN ${ }^{5}$, M. Juette ${ }^{9}$, R. Khan ${ }^{1}$, M. Kilic ${ }^{7}$, V. Knierim ${ }^{9}$, W. Laun ${ }^{10}$, R. LedereR $^{5}$, M. Lehmitz ${ }^{10}$, \\ R. Lenzen ${ }^{10}$, U. Mall ${ }^{10}$, K. K. Madsen ${ }^{11}$, H. Mandel $^{8}$, P. Martini $^{1,4}$, S. Mathur $^{1,4}$, K. Mogren ${ }^{1}$, P. Mueller $^{8}$, \\ V. Naranjo ${ }^{10}$, A. Pasquali ${ }^{10}$, K. Polsterer ${ }^{9}$, R. W. Pogge ${ }^{1,4}$, A. Quirrenbach $^{8}$, W. Seifert ${ }^{8}$, D. Stern $^{2}$, \\ B. Shappee ${ }^{1}$, C. Storz ${ }^{10}$, J. Van SAders ${ }^{1}$, P. Weiser $^{12}$, And D. Zhang ${ }^{1}$ \\ ${ }^{1}$ Department of Astronomy, The Ohio State University, 140 W. 18th Ave., Columbus, OH 43210, USA; rjassef@astronomy.ohio-state.edu \\ 2 Jet Propulsion Laboratory, California Institute of Technology, MS 169-530, 4800 Oak Grove Drive, Pasadena, CA 91109, USA \\ ${ }^{3}$ Dark Cosmology Centre, Niels Bohr Institute, University of Copenhagen, Juliane Maries Vej 30, 2100 Copenhagen, Denmark \\ 4 The Center for Cosmology and Astroparticle Physics, The Ohio State University, 191 West Woodruff Avenue, Columbus, OH 43210, USA \\ ${ }^{5}$ Max-Planck-Institut fuer Extraterrestrische Physik, Giessenbachstr., D-85748 Garching, Germany \\ ${ }^{6}$ Arkansas Center for Space and Planetary Sciences, University of Arkansas, Fayetteville, AR 72701, USA \\ ${ }^{7}$ Harvard-Smithsonian Center for Astrophysics, 60 Garden Street, Cambridge, MA 02138, USA \\ ${ }^{8}$ Landessternwarte, ZAH, Koenigstuhl 12, D-69117 Heidelberg, Germany \\ 9 Astron. Institut der Ruhr Univ. Bochum, Universitaetsstr. 150, D-44780 Bochum, Germany \\ ${ }^{10}$ Max-Planck-Institut fuer Astronomie, Koenigstuhl 17, D-69117 Heidelberg, Germany \\ ${ }^{11}$ California Institute of Technology, 1200 E. California Blvd, Pasadena, CA 91125, USA \\ ${ }^{12}$ Fachhochschule fuer Technik und Gestaltung, Windeckstr. 110, D-68163 Mannheim, Germany \\ Received 2010 September 6; accepted 2011 August 30; published 2011 November 9
}

\begin{abstract}
Using a sample of high-redshift lensed quasars from the CASTLES project with observed-frame ultraviolet or optical and near-infrared spectra, we have searched for possible biases between supermassive black hole $(\mathrm{BH})$ mass estimates based on the $\mathrm{C} \mathrm{IV}, \mathrm{H} \alpha$, and $\mathrm{H} \beta$ broad emission lines. Our sample is based upon that of Greene, Peng, \& Ludwig, expanded with new near-IR spectroscopic observations, consistently analyzed high signal-to-noise ratio $(\mathrm{S} / \mathrm{N})$ optical spectra, and consistent continuum luminosity estimates at $5100 \AA$. We find that $\mathrm{BH}$ mass estimates based on the full width at half-maximum (FWHM) of C IV show a systematic offset with respect to those obtained from the line dispersion, $\sigma_{l}$, of the same emission line, but not with those obtained from the FWHM of $\mathrm{H} \alpha$ and $\mathrm{H} \beta$. The magnitude of the offset depends on the treatment of the He II and Fe II emission blended with C IV, but there is little scatter for any fixed measurement prescription. While we otherwise find no systematic offsets between C IV and Balmer line mass estimates, we do find that the residuals between them are strongly correlated with the ratio of the UV and optical continuum luminosities. This means that much of the dispersion in previous comparisons of $\mathrm{C}$ IV and $\mathrm{H} \beta \mathrm{BH}$ mass estimates are due to the continuum luminosities rather than to any properties of the lines. Removing this dependency reduces the scatter between the UV- and optical-based BH mass estimates by a factor of approximately two, from roughly 0.35 to $0.18 \mathrm{dex}$. The dispersion is smallest when comparing the C IV $\sigma_{l}$ mass estimate, after removing the offset from the FWHM estimates, and either Balmer line mass estimate. The correlation with the continuum slope is likely due to a combination of reddening, host contamination, and object-dependent SED shapes. When we add additional heterogeneous measurements from the literature, the results are unchanged. Moreover, in a trial observation of a remaining outlier, the origin of the deviation is clearly due to unrecognized absorption in a low $\mathrm{S} / \mathrm{N}$ spectrum. This not only highlights the importance of the quality of the observations, but also raises the question whether cases like this one are common in the literature, further biasing comparisons between $\mathrm{C}$ IV and other broad emission lines.
\end{abstract}

Key words: galaxies: active - gravitational lensing: strong - quasars: emission lines

\section{INTRODUCTION}

It is thought that every massive galaxy has a supermassive black hole $(\mathrm{BH})$ at its center, and some physical properties of

\footnotetext{
* This work relies partly on observations of the Large Binocular Telescope (LBT). The LBT is an international collaboration among institutions in the United States, Italy, and Germany. LBT Corporation partners are The Ohio State University; The University of Arizona on behalf of the Arizona university system; Istituto Nazionale di Astrofisica, Italy; LBT Beteiligungsgesellschaft, Germany, representing the Max-Planck Society, the Astrophysical Institute Potsdam, and Heidelberg University; and The Research Corporation, on behalf of The University of Notre Dame, University of Minnesota, and University of Virginia.

13 NASA Postdoctoral Program Fellow.

14 DARK Fellow.
}

the $\mathrm{BH}$ appear to be tightly correlated with those of the galaxy. In particular, the mass of the central $\mathrm{BH}$ correlates well with the luminosity of the spheroidal component of the host (see, e.g., Marconi \& Hunt 2003; Graham 2007) and with its velocity dispersion (see, e.g., Ferrarese \& Merritt 2000; Gebhardt et al. 2000; Tremaine et al. 2002; Gültekin et al. 2009; Graham et al. 2011). Both of these properties of galaxies have physical scales a few orders of magnitude larger than the sphere of influence of the $\mathrm{BH}$, so mechanisms linking their properties are not immediately apparent. Theoretical models try to account for the correlation through co-evolution of the galaxy and its $\mathrm{BH}$, in which accretion induced by galaxy mergers regulates the BH's growth, and feedback from the accretion regulates the growth of the galaxy by quenching star formation and removing 
cold gas (e.g., Granato et al. 2004; Hopkins et al. 2005, 2006, 2008; Somerville et al. 2008; Shankar et al. 2009). However, the existence of these correlations does not necessarily imply co-evolutionary mechanisms, as some authors argue that they can be a simple consequence of mergers and the central limit theorem (Peng 2007, 2010; Jahnke \& Maccio 2011).

Direct measurements of $\mathrm{BH}$ masses in inactive galaxies are only possible for a small number of nearby objects because it is necessary, or at least desirable (see, e.g., Merritt \& Ferrarese 2001; Gültekin et al. 2009), to resolve the BH's sphere of influence in order to determine the $\mathrm{BH}$ mass from the kinematics of the stars and gas closest to it. Galaxies with active nuclei (AGNs) offer a completely different means of estimating $\mathrm{BH}$ masses at any distance. In particular, Type 1 AGNs show bright broad emission lines in their spectra produced by gas in the broad-line region (BLR), which is close to the central BH but outside the hot accretion disk. The large line widths are thought to arise from the Doppler broadening due to the orbital velocity of the gas around the $\mathrm{BH}$. Thus, measuring the mass of the central $\mathrm{BH}$ from the width of the broad lines is possible if the distance of the BLR from the BH is known.

This distance can be directly measured with the reverberation mapping (RM) technique (Blandford \& McKee 1982; Peterson 1993). This technique works by measuring the light travel time between the continuum and the broad-line emitting regions, which is derived from the time lag between changes in their respective luminosities. Unfortunately, the timescale over which appreciable variability is observed in AGNs increases with BH mass (e.g., Vanden Berk et al. 2004; Wilhite et al. 2008; Kelly et al. 2009; MacLeod et al. 2010), making it difficult (i.e., more time intensive) to apply RM to the luminous QSOs that possess the most massive BHs. For example, MacLeod et al. (2010) find that for a typical quasar with $M_{\mathrm{BH}}=10^{8} M_{\odot}$ (typical magnitude of $M_{i} \approx-23 \mathrm{mag}$ ), the rest-frame timescale, $\Delta t$, required to reach an rms variability amplitude of $0.1 \mathrm{mag}$ is approximately 45 days, while for a quasar with $M_{\mathrm{BH}}=10^{9} M_{\odot}$ $\left(M_{i} \approx-25.5 \mathrm{mag}\right), \Delta t$ is approximately 125 days. This is further complicated by the time dilation due to the higher redshift of these rare objects. It has been shown, however, that the distance from the $\mathrm{BH}$ to the BLR correlates well with the continuum luminosity of the AGN (see, e.g., Kaspi et al. 2000, 2005; Bentz et al. 2006, 2009; Zu et al. 2011). Given this correlation, $\mathrm{BH}$ masses can be estimated for distant broadline quasars for which the RM technique is not reasonably applicable. Masses estimated in this way are usually referred to as single epoch (SE) BH mass estimates.

Because it is generally easier to obtain optical rather than UV or IR spectra, SE BH masses are typically estimated from the $\mathrm{H} \beta$ and $\mathrm{H} \alpha$ broad emission lines and the continuum luminosity at $5100 \AA$ at low redshifts $(z \lesssim 0.7)$. The overlap with RM targets has allowed for very accurate calibration of these SE mass estimators (Collin et al. 2006; Vestergaard \& Peterson 2006, hereafter VP06; McGill et al. 2008). At high redshift, however, these emission lines are shifted into the IR, and most mass estimates are then based upon the UV Mg II $\lambda 2798$ and $\mathrm{C}$ IV $\lambda 1549$ broad emission lines and the continuum luminosities at $3000 \AA$ for $\mathrm{Mg}$ II and $1450 \AA$ or $1350 \AA$ for $\mathrm{C}$ IV. Unlike the Balmer emission lines, these UV lines lack large local calibration samples because of the difficulty of obtaining UVbased RM measurements. Onken \& Kollmeier (2008) have argued that the $\mathrm{Mg}$ II line can provide accurate mass estimates, but that there is a small, but significant, dependence on the Eddington ratio of the AGN. CIV, on the other hand, is not thought to have this bias, and VP06 have calibrated a C IV-based mass estimator based on local RM AGNs using space-based UV spectra. However, there are still concerns about whether the C IV velocity widths are attributed solely to gravity or if there are bulk flows due to winds of ejected material, and the impact of these effects on the accuracy of C IV-based BH mass estimates is still debated. For example, C IV is typically slightly displaced in wavelength (usually blueshifted) with respect to the rest of the quasar emission lines (see, e.g., Gaskell 1982; Tytler \& Fan 1992; Richards et al. 2002) and frequently shows broad absorption features (e.g., Weymann et al. 1981) and strong line asymmetries correlated with quasar properties (e.g., Wilkes 1984; Richards et al. 2002; Leighly \& Moore 2004).

The simplest approach to test the reliability of C IV mass estimates is to systematically compare them to Balmer line estimates for the same sources (see, e.g., Dietrich et al. 2009). High-redshift lensed quasars are some of the best targets for such tests. Generally, the problem is that the high redshift makes it easy to observe the $\mathrm{C}$ IV line, but the better calibrated $\mathrm{H} \alpha$ and $\mathrm{H} \beta$ lines lie in the near-IR, where it is difficult to observe them. Magnification increases the apparent brightness of the lensed quasars, and also, because their observed brightness is not uniquely determined by their intrinsic luminosity and distance, it helps to mitigate any Eddington biases in the sample or, in other words, it makes objects in the sample unlikely to be preferentially brighter than average for their BH mass.

In a recent work, Greene et al. (2010, hereafter GPL10) presented near-IR spectral observations for a sub-sample of lensed quasars from the CfA-Arizona Space Telescope LEns Survey (CASTLES) of gravitational lenses (Falco et al. 2001) whose C IV or Mg II BH masses had been estimated in a previous work by Peng et al. (2006). GPL10 measured, whenever possible, the full width at half-maximum (FWHM) of the $\mathrm{H} \beta$ and $\mathrm{H} \alpha$ emission lines of these objects and found no systematic biases between $\mathrm{BH}$ masses estimated from these lines and those estimated from C IV. Their sample, however, did not cover a large enough range in $\mathrm{BH}$ mass to decide whether there was a mass-dependent slope to the relation between the masses. This comparison also suffered from the fact that Peng et al. (2006) lacked access to the original UV/optical spectra for many targets and frequently had to rely on the printed spectra in published papers to measure line widths.

In this work, we start from the sample of GPL10 and attempt to improve on both of these issues. First, we add Balmerline-based $\mathrm{BH}$ mass estimates for the lens SDSS1138+0314 and make revised estimates based on new, higher signal-tonoise ratio (S/N) spectra of HS0810+2554 and SBS0909+532. We obtained near-IR observations for SDSS1138+0314 and HS0810+2554 using the newly commissioned Large Binocular Telescope (LBT) NIR Spectrograph Utility Camera and Integral Field Unit (LUCIFER; Seifert et al. 2003; Ageorges et al. 2010), while for SBS0909+532 we use the UV through IR observations of Mediavilla et al. (2011). Second, we made consistent C IV $\mathrm{BH}$ mass estimates from high $\mathrm{S} / \mathrm{N}$ spectra using the original observations analyzed by Peng et al. (2006), other published or unpublished spectra, or new spectra for all targets in the sample. Finally, we obtained continuum luminosities at $5100 \AA$ for all objects in the sample in a consistent manner. This allows us to include the lenses SDSS0246-0825, HS0810+2554, and Q2237+030, which were excluded by GPL10. With these additions we expand the sample of GPL10 with both C IV and Balmer line mass estimates from 7 to 12 quasars and the mass range covered by approximately 0.5 dex. In Section 2, we 
Table 1

Lens Magnifications and Continuum Luminosities

\begin{tabular}{|c|c|c|c|c|c|c|c|c|c|c|}
\hline \multirow[t]{2}{*}{ Object } & \multirow[t]{2}{*}{ Ref. } & \multirow[t]{2}{*}{$z$} & \multicolumn{4}{|c|}{ Image Magnification } & \multirow{2}{*}{$\begin{array}{c}m_{H}^{\mathrm{a}} \\
\text { (mag) }\end{array}$} & \multicolumn{3}{|c|}{$\overline{l o g}\left(\lambda L_{\lambda} /\right.$ erg s$\left.^{-1}\right)$} \\
\hline & & & A & B & $\mathrm{C}$ & $\mathrm{D}$ & & $1350 \AA$ & $1450 \AA$ & $5100 \AA$ \\
\hline Q0142-100 & $\mathrm{a}$ & 2.72 & 3.3 & 0.4 & $\ldots$ & $\ldots$ & 16.56 & 46.83 & 46.76 & 46.27 \\
\hline SDSS0246-0825 & $\mathrm{a}$ & 1.69 & 26.9 & 8.9 & $\ldots$ & $\ldots$ & 20.39 & $\ldots$ & 44.53 & 44.59 \\
\hline HS0810+2554 & $\ldots$ & 1.51 & 47.2 & 51.1 & 13.5 & 7.7 & 18.72 & $\ldots$ & 44.44 & 44.84 \\
\hline SBS0909+532 & $\mathrm{b}$ & 1.38 & 1.7 & 1.5 & $\ldots$ & $\ldots$ & 15.18 & 46.08 & 46.05 & 46.31 \\
\hline Q0957+561 & $\mathrm{c}$ & 1.41 & 3.1 & 1.7 & $\ldots$ & $\ldots$ & 16.51 & 46.31 & 46.25 & 45.79 \\
\hline HE1104-1805 & $\mathrm{d}$ & 2.32 & 16.2 & 2.3 & $\ldots$ & $\ldots$ & 18.52 & 46.15 & 46.09 & 45.38 \\
\hline PG1115+080 & $\mathrm{a}$ & 1.72 & 19.6 & 18.7 & 3.2 & 4.9 & 19.13 & $\ldots$ & 45.47 & 44.93 \\
\hline SDSS1138+0314 & $\mathrm{e}$ & 2.44 & 7.3 & 3.7 & 5.2 & 6.9 & 20.65 & 44.83 & 44.77 & 44.81 \\
\hline $\mathrm{H} 1413+117$ & $\mathrm{a}$ & 2.55 & 8.2 & 6.8 & 6.8 & 3.4 & 18.05 & 45.73 & 45.78 & 45.63 \\
\hline B1422+231 & $\mathrm{f}$ & 3.62 & 6.6 & 8.2 & 4.3 & 0.3 & 16.55 & 46.83 & 46.74 & 46.42 \\
\hline FBQ1633+3134 & $\ldots$ & 1.52 & 2.7 & 0.7 & $\ldots$ & $\ldots$ & 16.85 & 45.65 & 45.64 & 45.72 \\
\hline $\mathrm{Q} 2237+030$ & $\mathrm{~g}$ & 1.69 & 4.9 & 4.3 & 2.2 & 4.1 & 16.83 & $\ldots$ & 45.53 & 45.98 \\
\hline
\end{tabular}

Notes. Literature UV/optical spectra obtained from the following references: (a) SDSS DR7 Abazajian et al. 2009; (b) Mediavilla et al. (2011); (c) Goicoechea et al. 2005; (d) Wisotzki et al. 1995; (e) Eigenbrod et al. 2006; (f) Tonry 1998; (g) Eigenbrod et al. 2008.

${ }^{\text {a }}$ Unmagnified quasar $H$-band magnitudes.

describe the sample of gravitationally lensed quasars we use in this study as well as our observations. In Section 3, we describe the methods we use to measure emission line velocity widths and their uncertainties, the continuum luminosities of the quasars, and the SE BH masses. In Section 4, we compare the different mass estimates we have derived and determine the possible biases we measure between them while in Section 5 we expand our results using a heterogeneous sample of measurements from other studies. In Section 6, we summarize the conclusions. In the Appendix, we discuss individual objects in detail. We use a standard $\Lambda$ CDM cosmology with $\Omega_{M}=0.3, \Omega_{\Lambda}=0.7$, and $H_{0}=73 \mathrm{~km} \mathrm{~s}^{-1} \mathrm{Mpc}^{-1}$ throughout the paper.

\section{THE SAMPLE OF LENSED QSOS}

We selected 12 lensed quasars from the CASTLES survey with high quality UV/optical, typically ground-based, spectra of C IV and either published near-IR spectra of the Balmer lines or IR magnitudes bright enough to obtain such spectra. The targets are listed in Table 1 . All 12 objects have been observed by CASTLES with the Hubble Space Telescope (HST) in the $V$ (F555W), $I(\mathrm{~F} 814 \mathrm{~W})$, and $H(\mathrm{~F} 160 \mathrm{~W})$ bands, except for B1422+231, which was not observed in $I$.

We start from the sample of GPL10, who observed most of these lensed quasars in the near-IR with the Triplespec spectrograph at the Apache Point Observatory. The wavelength range of these spectra is $0.95-2.46 \mu \mathrm{m}$ with $R=3500$, and either the $\mathrm{H} \beta$ or $\mathrm{H} \alpha$ (or both) emission line is observable in one of the atmospheric windows. Although GPL10 considered objects with a large span of redshift and reddening, we limit our sample to objects with sufficiently high redshift and small enough reddening for $\mathrm{C}$ IV emission to be observable in ground based UV/optical spectra. ${ }^{15}$ GPL10 presented FWHM velocity width measurements for all the objects in their sample but did not present $\mathrm{BH}$ mass estimates for three of them. For these three lensed QSOs, SDSS0246-0825, HS0810+2554, and Q2237+030, we have measured the continuum luminosity and estimated BH masses so we can include them in our sample.

\footnotetext{
15 Peng et al. (2006) mistakenly quote a C IV BH mass estimate for the lens J1004+1229 that, in fact, corresponds to the lens SDSS1004+4112 (C. Y. Peng 2010, private communication). This error was propagated into the analysis of GPL10. The quasar in $\mathrm{J} 1004+1229$ is highly reddened and it is not possible to see its C IV emission in a UV/optical spectrum.
}
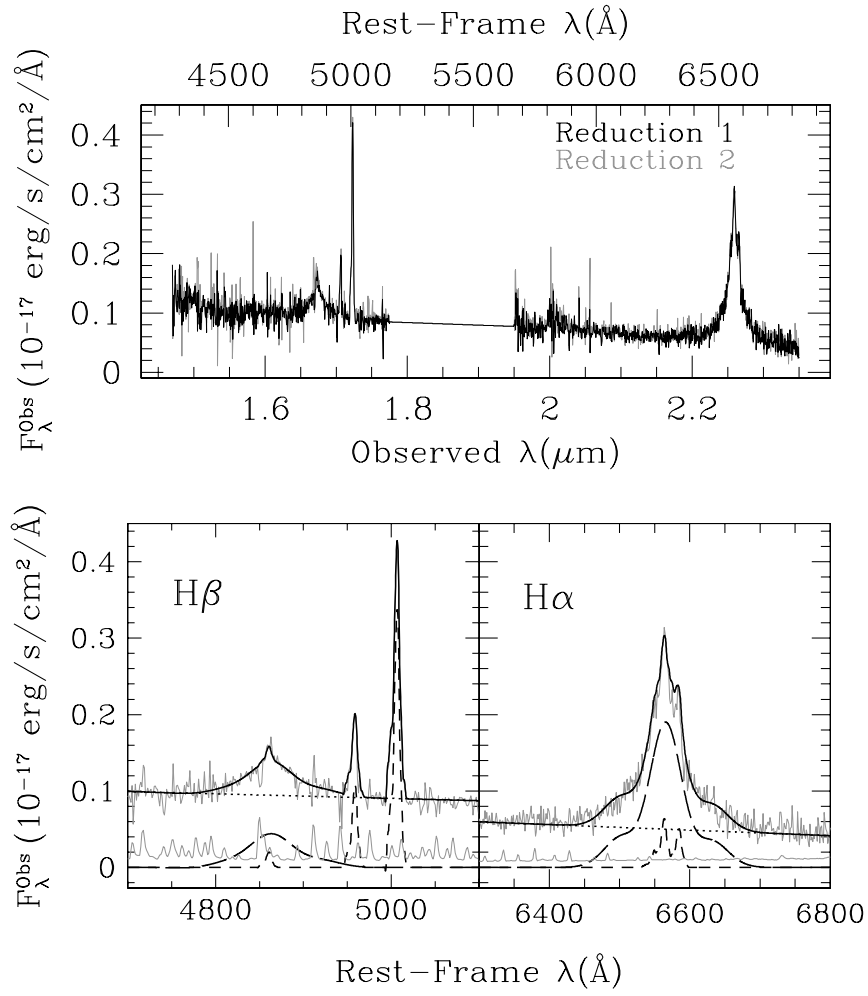

Figure 1. Top: LUCIFER $H$ - and $K$-band spectra of SDSS1138+0314. The black solid line shows the spectrum obtained by performing the sky subtraction with the median combination of the sky frames while the gray line shows that obtained by using the modified version of the COSMOS software described in the text. Bottom left: spectral region around $\mathrm{H} \beta$. Overlaid on top are the best-fit continuum (black dotted line) and narrow (black short-dashed line) and broadline components (black long-dashed line), as well as their sum (black solid line) and the error spectrum (thin gray solid line). Bottom right: same as bottom left but for $\mathrm{H} \alpha$.

We obtained near-IR spectra in the $H$ and $K$ band for SDSS1138+0314 (Figure 1) and in the $J$ band for HS0810+2554 (Figure 2) with the LBT LUCIFER spectrograph. The first was obtained as part of the LUCIFER science demonstration time and is discussed here, while the second was a target of a separate project to be presented by K. Mogren et al. (2011, in preparation). We also analyzed the near-IR $J$ - and $H$-band 


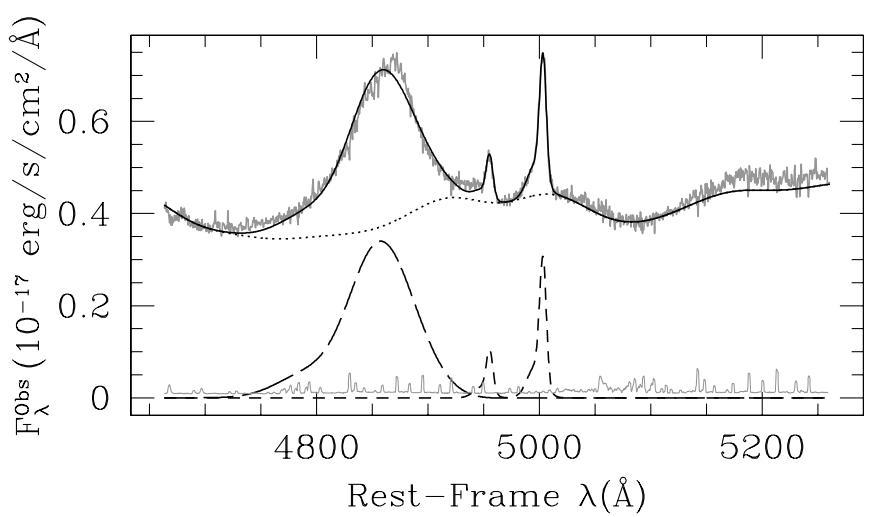

Figure 2. LUCIFER $J$-band spectrum of HS0810+2554 (gray solid line). Overlaid are the best-fit continuum and Fe II emission (black dotted line), narrow line emission (black short-dashed line), broad-line component (black long-dashed line), and their sum (black solid line), as well as the error spectrum (thin gray solid line).

observations of SBS0909+532 presented by Mediavilla et al. (2011), shown in Figure 3.

\subsection{LUCIFER Observations of SDSS1138+0314}

We obtained a near-infrared spectrum of SDSS1138+0314 using the new LUCIFER instrument at the LBT during its science demonstration time. LUCIFER is a near-infrared spectrograph and imager with an overall wavelength range of $0.85-2.5 \mu \mathrm{m}$. We observed SDSS1138+0314 in the longslit mode with the OrderSep filter, a 0.5 slit, the 200_H+K grating, and the N1.8 camera for a total integration time of 840 s over seven dithered exposures during the night of UTC 2010 January 4. This configuration gives an effective wavelength range of 1.49-2.4 $\mu \mathrm{m}$, which includes both the $H$ and $K$ bands, with a resolving power of 1880 at $H$ and 2570 at $K$. The slit was oriented to include images $\mathrm{A}$ and $\mathrm{C}$ of the lensed quasar, as well as part of the lens galaxy. No emission from the lens galaxy is detected in our data. The B9V star HIP 33350 was observed with the same configuration, except for a change in slit width from 0.5 to $1^{\prime \prime}$, and was used to correct the spectrum of SDSS1138+0314 for telluric absorption features. The difference in resolution caused by the different slit widths degrades our telluric corrections, but has little consequence for measuring the width of broad emission lines. We estimated that the seeing was $\sim 0$ '.8 during the observations.

We reduced the data using standard IRAF packages in combination with the IDL task xtellcor_general of Vacca et al. (2003) for the telluric absorption corrections. We performed a two-dimensional wavelength calibration on each of the seven exposures using the sky emission lines and built a sky frame by median combining them. The sky frame was then used to remove the sky from each exposure before extracting the spectrum. We also did an alternate sky subtraction of the spectra using a version of the COSMOS software modified to work on LUCIFER data. This software, designed for reduction of spectral observations with IMACS (Dressler et al. 2006) and LDSS-3 (upgraded from LDSS-2; Allington-Smith et al. 1994) on the Magellan telescopes, follows the procedures of Kelson (2003). It produces an accurate model of the sky emission by creating a sub-pixel resolution map of the sky line profiles using the full extent of the lines in the spectrum coupled with a model of the optical distortions. Both extractions of the spectra yield equivalent results and both are shown in Figure 1. While in principle we could use the telluric standard to perform an
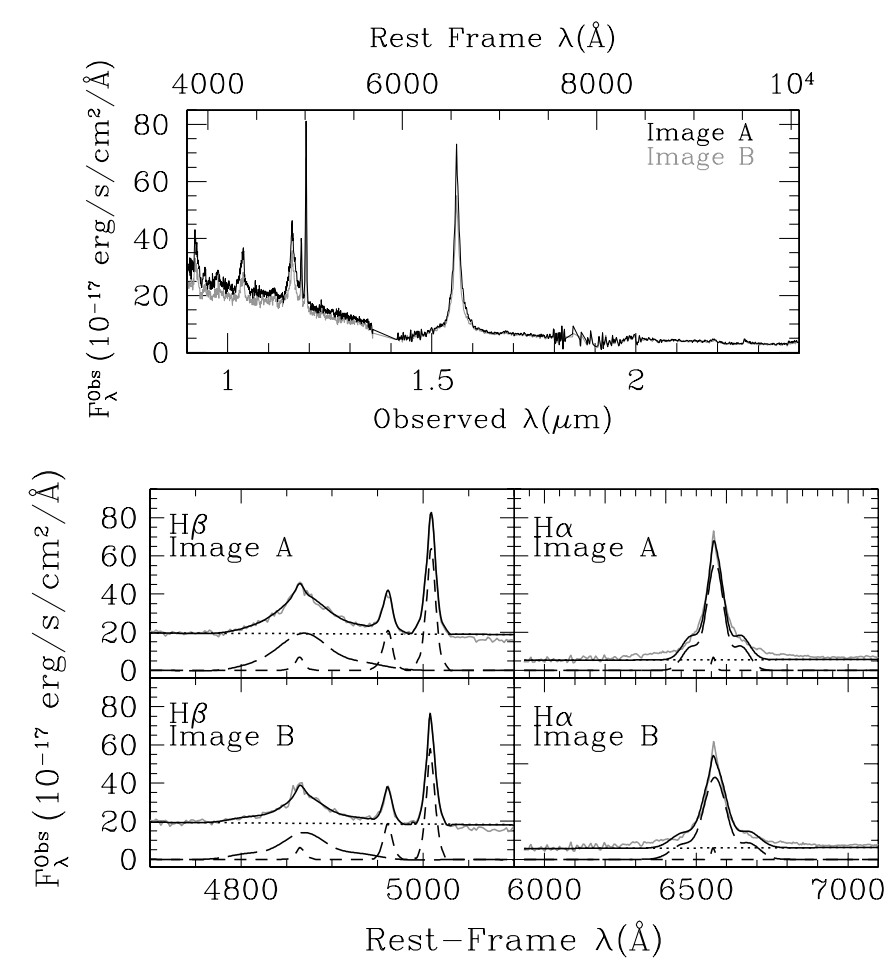

Figure 3. LIRIS near-IR spectra of images A and B of SBS0909+532 obtained by Mediavilla et al. (2011). The top panel shows the complete spectrum while the bottom four panels show the spectral regions around $\mathrm{H} \alpha$ and $\mathrm{H} \beta$ of each quasar image. Overlaid on top are the best-fit continuum and narrow- and broadline components, as well as their overall sum, using the same line styles as in Figure 1.

absolute flux calibration, it is hard to model the slit losses, especially considering the difference in the slit widths. Instead, the flux calibration was performed by convolving the spectrum corrected for telluric absorption with the NICMOS F160W filter curve and matching it to the estimated de-magnified absolute magnitude of the quasar from the CASTLES HST imaging of this lens (see Section 3.3 for details on the lens magnification). The blue edge of the LUCIFER SDSS1138+0314 spectrum is somewhat redder than the blue edge of the F160W band, so we extended the observed spectrum using the AGN spectral energy distribution (SED) template of Assef et al. (2010) assuming no reddening. Note that the HST NICMOS observations were obtained on UTC 2003 November 6, approximately 6 years before the LUCIFER observations, so we attempt to correct for the intrinsic variability of the quasar. However, this is typically not an important correction (see Section 3.3). We use the $R$-band light curves obtained with the SMARTS $1.3 \mathrm{~m}$ telescope for a gravitational lens monitoring project (see Morgan et al. 2010). These data show that the quasar intrinsically brightened by $56 \% \pm 17 \%$ between UTC 2004 February 3 and UTC 2010 January 9. We assume that no significant variability occurred between the HST NICMOS and the first SMARTS observations and between the LUCIFER and the last SMARTS observations.

From an optical spectrum of SDSS1138+0314, Eigenbrod et al. (2006) estimated a redshift of $z=2.438$ for the quasar, while SDSS provides $z=2.4427 \pm 0.0014$. Using the narrow component of $\mathrm{H} \alpha$ and the [O III] $\lambda \lambda$ 4959, 5007 emission lines, we obtained $z=2.4417$, consistent with SDSS. We did not use the $[\mathrm{N}$ II] lines or the narrow component of $\mathrm{H} \beta$ as they could not be centroided accurately because of blending with the broad $\mathrm{H} \alpha$ and $\mathrm{H} \beta$ profiles, respectively. 

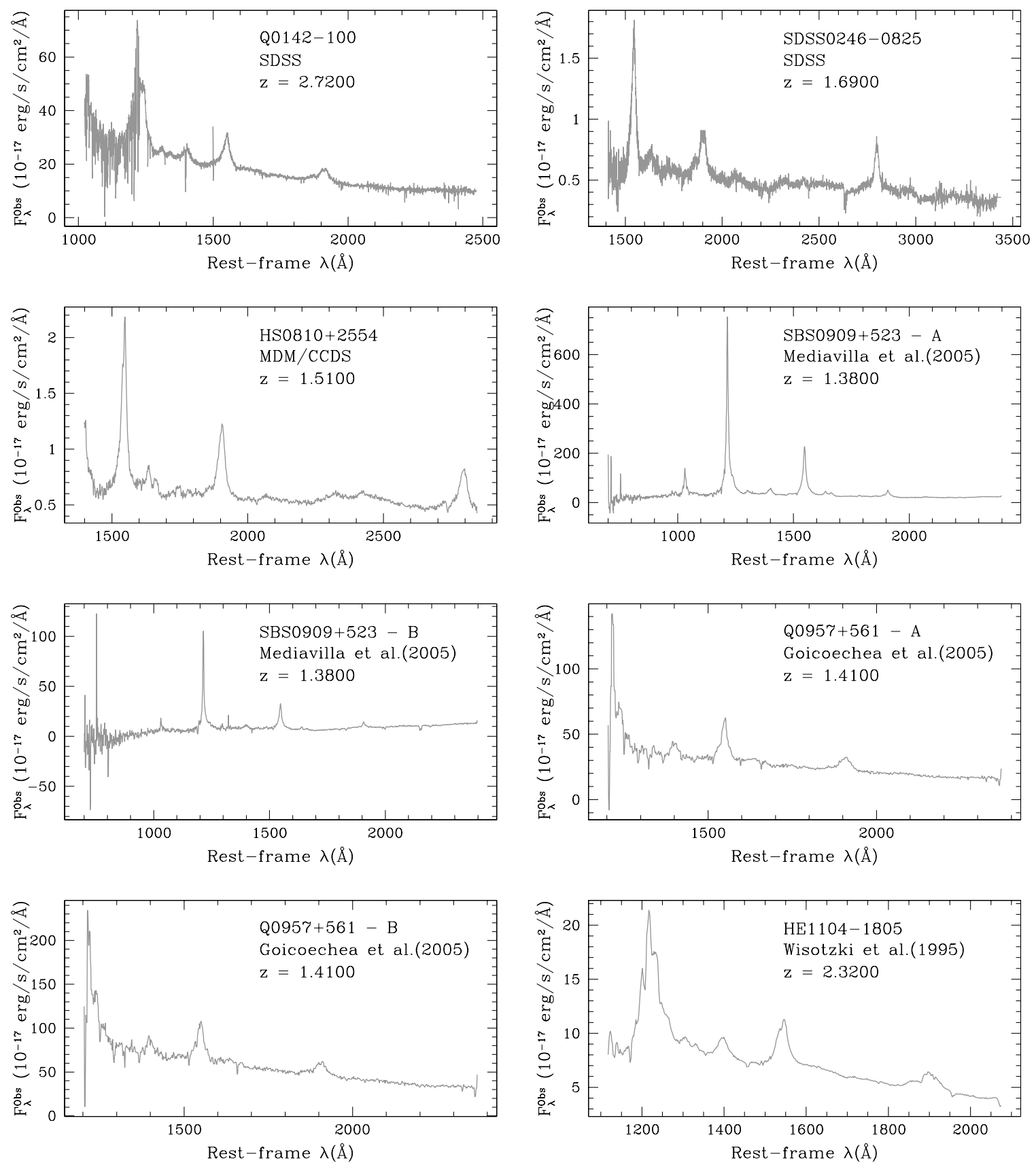

Figure 4. UV/optical spectra of all objects used in this study. For each object the panel shows the full spectrum, the source from which it was obtained, and its redshift.

\subsection{UV/Optical Spectra}

For most of the GPL10 sample, as well as for SDSS1138+0314, we found suitable high S/N optical spectroscopic observations in the literature that the owners kindly made available for this study (see Table 1 for the references, where applicable, and the Appendix for details on each object). When needed, we performed an absolute flux calibration using photometry from several different sources, as this was not always required for the science goals of the original project. All the UV/optical spectra compiled from the literature are shown in Figure 4.

We could not locate suitable optical spectra for HS0810+2554 and FQB1633+3134. Both objects were observed by the SDSS spectroscopic survey, but these spectra did not have high enough $\mathrm{S} / \mathrm{N}$ to provide accurate line-width measurements with good continuum subtraction. We obtained new optical spectra of these objects using the MDM observatory $2.4 \mathrm{~m}$ Hiltner telescope with the Boller \& Chivens CCD Spectrograph ${ }^{16}$ (CCDS). HS0810+2554 was observed on UTC 2010 February 24 with a grating center of $5300 \AA$ and was flux calibrated using the standard star Feige 34. FBQ1633+3134 was observed on UTC 2010 March 21 and UTC 2010 March 22 with a grating center of $4700 \AA$ and was flux calibrated using the standard star Feige 98. Absolute fluxes were obtained for both objects by performing a cross-calibration between SDSS $g$-band photometry of other objects in the field and $g$-band photometric observations with the RETROCAM instrument (Morgan et al. 2005) obtained on UTC 2010 March 6 and UTC 2010 March 22 for HS0810+2554 and

\footnotetext{
16 http://www.astronomy.ohio-state.edu/MDM/CCDS/
} 

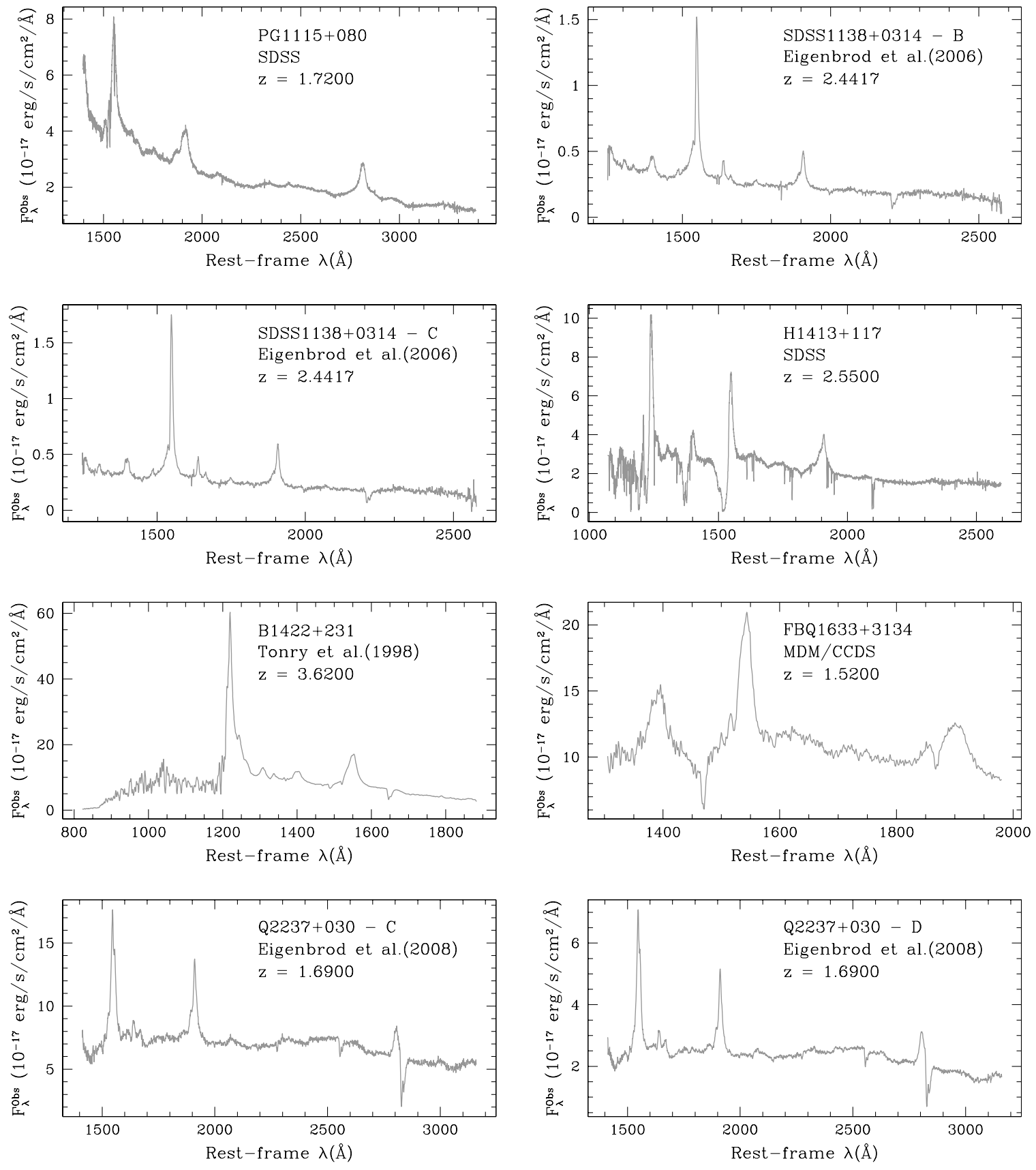

Figure 4. (Continued)

FBQ1633+3134, respectively. The reduced spectra are shown in Figure 4.

\section{MODELS AND MEASUREMENTS}

In this section, we briefly discuss the methods we use to measure the line widths and estimate the $\mathrm{BH}$ masses from the optical and near-IR spectra.

\subsection{Line-width Measurements}

There is no standard prescription for measuring the linewidth characterizations of the broad C IV emission line in QSOs. While for other emission lines this may not be a significant source of uncertainties, there is a shelf-like emission feature redward of C IV that blends with the line profile and is created by a combination of broad He II $\left.\lambda 1640, \mathrm{O}_{\mathrm{III}}\right] \lambda 1663$, and a feature of unknown origin at $1600 \AA$ usually referred to as the $\lambda 1600$ feature (Laor et al. 1994; Marziani et al. 1996; Fine et al. 2010). While the $\lambda 1600$ feature is commonly thought to correspond to Fe II, Fine et al. (2010) argue that this cannot account for all the observed flux, yet it is also unlikely that $\mathrm{C}$ IV can reach large enough velocities to produce the feature. Different prescriptions for modeling the blended emission can have significant effects on line-width estimates (Denney et al. 2009; Fine et al. 2010), so it is important to explore how these affect our results. Fine et al. (2010) explored three different and widely used approaches and their effects on the C IV width measurements. The three prescriptions are (1) to assume that the $\lambda 1600$ feature corresponds to C IV emission and therefore remove only the He II $\lambda 1640$ and O III] $\lambda 1663$ contributions, (2) to assume that the $\lambda 1600$ feature belongs to a different species from C IV and so removing its contribution along with that of 
Table 2

C Iv Emission Line and Continuum Region Boundaries

\begin{tabular}{|c|c|c|c|c|c|c|c|c|}
\hline \multirow[b]{2}{*}{ Object } & \multirow[b]{2}{*}{$\mathrm{S} / \mathrm{N}^{\mathrm{a}}$} & \multicolumn{3}{|c|}{ Prescription A } & \multicolumn{3}{|c|}{ Prescription B } & \multirow[b]{2}{*}{$\begin{array}{l}\text { Res } \\
(\AA)\end{array}$} \\
\hline & & $\begin{array}{c}\text { Blue Cont. } \\
(\AA)\end{array}$ & $\begin{array}{l}\text { Red Cont. } \\
(\AA)\end{array}$ & $\begin{array}{c}\text { Broad Line } \\
(\AA)\end{array}$ & $\begin{array}{c}\text { Blue Cont. } \\
(\AA)\end{array}$ & $\begin{array}{c}\text { Red Cont. } \\
(\AA)\end{array}$ & $\begin{array}{c}\text { Broad Line } \\
(\AA)\end{array}$ & \\
\hline Q0142-100 & 45 & $5401-5468$ & $6250-6343$ & $5570-5954$ & $5440-5500$ & $5900-5960$ & $5500-5900$ & 2.6 \\
\hline SDSS0246-0825 & 17 & $3980-4030$ & $4519-4586$ & $4028-4306$ & $3980-4030$ & $4250-4300$ & $4050-4250$ & 2.6 \\
\hline HS0810+2554 & 19 & $3610-3690$ & $4217-4280$ & $3758-4018$ & $3620-3685$ & 3985-4005 & $3715-3975$ & 15.2 \\
\hline SBS0909+532-A & 71 & $3420-3470$ & $4070-4120$ & $3564-3810$ & $3420-3470$ & $3820-3850$ & $3560-3820$ & 3.5 \\
\hline Q0957+561-A & 66 & $3434-3543$ & 4049-4109 & $3609-3858$ & $3550-3600$ & $3810-3830$ & $3655-3805$ & 3.5 \\
\hline Q0957+561-B & 28 & $3434-3543$ & 4049-4109 & $3609-3858$ & $3550-3600$ & $3810-3830$ & $3655-3805$ & 3.5 \\
\hline HE1104-1805 & 221 & $4731-4880$ & $5578-5661$ & $4971-5314$ & $4870-4910$ & $5300-5340$ & $4970-5290$ & $\ldots$ \\
\hline PG1115+080 & 86 & $3897-4020$ & $4595-4663$ & $4095-4378$ & $4000-4031$ & $4415-4455$ & $4085-4415$ & 2.2 \\
\hline SDSS1138+0314-B & 78 & 4904-5059 & $5782-5868$ & $5153-5509$ & $4974-5020$ & $5520-5540$ & $5020-5520$ & $\ldots$ \\
\hline SDSS1138+0314-C & 47 & 4904-5059 & $5782-5868$ & $5153-5509$ & $4974-5020$ & $5520-5540$ & $5020-5520$ & $\ldots$ \\
\hline $\mathrm{H} 1413+117$ & 43 & $5073-5233$ & $5981-6070$ & $5331-5698$ & $5140-5190$ & $5630-5680$ & $5210-5615$ & 2.3 \\
\hline FBQ1633+3134 & 52 & $3350-3412$ & $4234-4297$ & $3773-4034$ & $3350-3412$ & $4020-4060$ & $3795-3980$ & 13.0 \\
\hline Q2237+030-C & 36 & $3840-3962$ & $4528-4595$ & $4035-4314$ & $3870-3910$ & $4275-4315$ & $4040-4265$ & $\ldots$ \\
\hline Q2237+030-D & 54 & $3840-3962$ & $4528-4595$ & $4035-4314$ & $3870-3910$ & $4275-4315$ & $4040-4265$ & . \\
\hline
\end{tabular}

Note. ${ }^{\text {a }}$ The $\mathrm{S} / \mathrm{N}$ quoted is the $\mathrm{S} / \mathrm{N}$ per pixel averaged over all continuum regions listed for each object.

the other two components on the shelf, and (3) to fit the $\lambda 1600$ feature as part of the continuum (see Fine et al. 2010 for details on each prescription). While Fine et al. (2010) select prescription (2) as their preferred method, in large part because it produces symmetric C IV profiles, it is hard to apply this approach to low S/N data (see Fine et al. 2010 for details). Moreover, it is not guaranteed to produce more accurate $\mathrm{BH}$ masses than the other two prescriptions. The simple prescription of (3) produces line-width characterizations that are systematically smaller than prescription (2) but with very low dispersion between individual measurements, while (1) produces estimates with a larger scatter relative to (2) but without a systematic offset. The differences between the prescriptions are smallest for FWHM and largest for the line dispersion, $\sigma_{l}$.

Based, in part, on these issues, we considered two different prescriptions for removing the continuum and blended emission from the CIV emission line profile. Both prescriptions are amenable to large-scale automated use. The first prescription, which we will refer to as prescription A, is very similar to that used by VP06, where the shelf feature redward of CIV is considered part of the $\mathrm{C}$ IV line profile, but only the region within $\pm 10,000 \mathrm{~km} \mathrm{~s}^{-1}$ of the peak is considered. The continuum is fit by linearly interpolating between the two continuum windows in the wavelength ranges $1425-1470$ and $1680-1705 \AA$. When these continuum windows were affected by absorption, we slightly shifted them as detailed in Table 2 . Our continuum fitting is in principle different from that of VP06, who considered five different continuum windows and then fit a power law to them, but the differences of the measured line widths are not significant and our approach requires a much smaller wavelength range for the spectra. In this prescription, He II and $\mathrm{O}$ III] emission are not explicitly removed, but this has negligible effects due to the limit on the velocity range, making it analogous to prescription (1) of Fine et al. (2010). The second prescription, B, is analogous to prescription (3) of Fine et al. (2010), as we fit the $\lambda 1600$ feature as part of the continuum. It only differs in that the red continuum region is chosen to match the minimum between $\mathrm{C}$ IV and the $\lambda 1600$ feature. In general, prescription A will lead to broader estimates of the C IV line width than prescription B.
The observed wavelength continuum windows for each object and prescription are listed in Table 2 . The $\mathrm{C}$ IV emission line flux was then measured above the fit continuum and between the emission line wavelength regions listed in Table 2. In addition, for objects that showed mild absorption features, bad pixels, and/or significant night sky line residuals, we used a low-order polynomial (i.e., first, second, or third order depending on the size and location of the feature) to interpolate across the feature before measuring the line widths. Details for the individual targets are given in the Appendix. We did not attempt to remove any narrow-line emission from CIV $\lambda 1549$, since this line is typically very weak and cannot be reliably isolated (Wills et al. 1993, although see Sulentic et al. 2007), and the separate lines of the CIV doublet are unresolved in AGN spectra (see VP06 and references therein for further discussion). We characterized the line width by both its FWHM and line dispersion ( $\sigma_{l}$, the second moment of the line profile). The widths were measured directly from the actual or interpolated spectrum (except where noted below and in the Appendix) following the procedures described by Peterson et al. (2004).

We also fit the original or interpolated line profiles with a sixth-order Gauss-Hermite (GH) polynomial, because making functional fits to emission-line profiles is a common way of mitigating the effects of low $\mathrm{S} / \mathrm{N}$ on line-width measurements (see, e.g., Woo et al. 2007; McGill et al. 2008, for similar approaches). The GH polynomials we fit utilize the normalization of van der Marel \& Franx (1993) and the functional forms of Cappellari et al. (2002). We then use a Levenberg-Marquardt least-squares fitting procedure to determine the best-fitting coefficients. We measured the widths of these line profile models using the same software as was used to measure widths directly from the data (see Peterson et al. 2004). Ultimately we only used the results from the line profile models for PG1115+080 (see the Appendix). Instead, these fits were primarily used to determine uncertainties in our width measurements as described in Section 3.2. The continuum and the GH fits to the C IV line profiles are shown in Figure 5 for both prescriptions. In the cases of SDSS1138+0314 and SBS0909+532, reasonable fits could not be achieved because of the extremely high $\mathrm{S} / \mathrm{N}$ and 


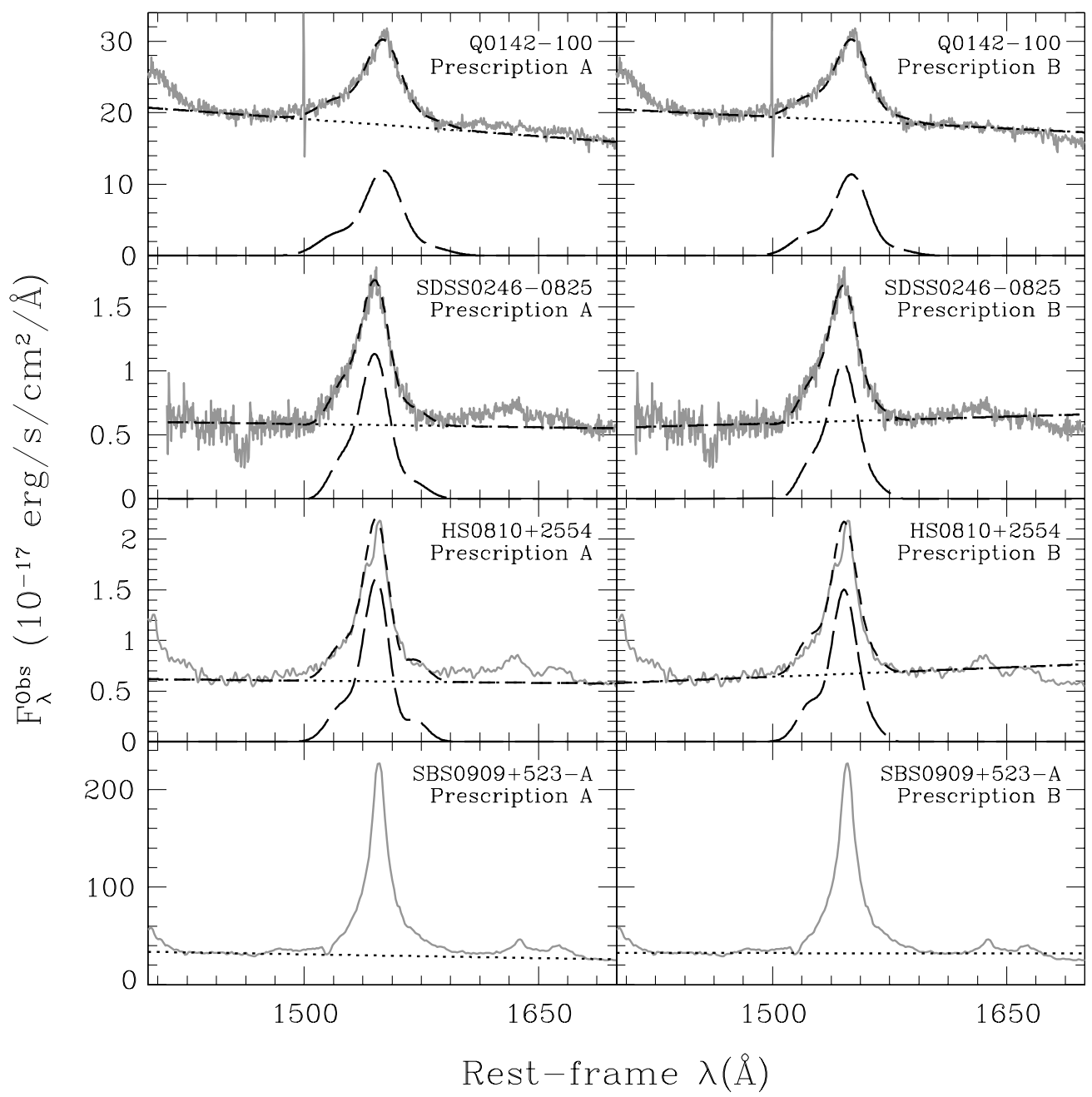

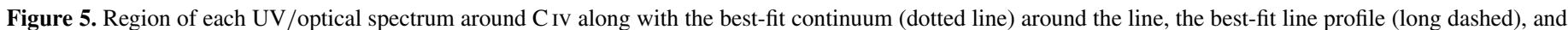

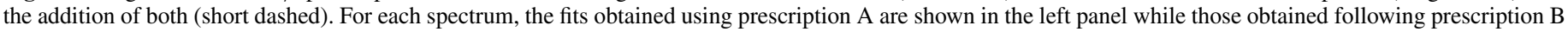
are shown in the right panel. We could not obtain good fits for SDSS1138+0314 and SBS0909+532 C IV lines (see the Appendix for details).

peculiar shape of these line profiles (a very narrow peak with broad base; see the Appendix). No fits are shown for these objects.

Both the FWHM and line dispersion, $\sigma_{l}$, measurements of the C IV $\lambda 1549$ emission line are listed in Table 3 for all objects in our sample for both prescriptions. We have corrected the widths for spectral resolution effects following Peterson et al. (2004), when possible, using the resolutions given in Table 2. Except for PG1115+080, we utilize the line widths measured directly from the data (interpolated across gaps where noted) for the subsequent $\mathrm{BH}$ mass calculations. For objects with multiple spectra of the individually lensed images we averaged their line widths. Our C IV $\lambda 1549$ widths are smaller than those given by GPL10 for the objects in which we used both SDSS spectra (Q0142-100, SDSS0246-0825, PG1115+080, and $\mathrm{H} 1413+117)$. The likely origin of the discrepancy is that GPL10 fit a narrow line component as part of the C IV profile, which would naturally yield larger FWHM values. We note, however, that GPL10 do not use their SDSS line-width measurements to estimate $\mathrm{BH}$ masses in their analysis, but always use those determined by Peng et al. (2006). The lens HE1104-1805 is the only object in the sample for which we use the same optical spectrum as Peng et al. (2006), that of Wisotzki et al. (1995), and we find an FWHM that is smaller by $260 \mathrm{~km} \mathrm{~s}^{-1}$ compared to our measured uncertainty of $50 \mathrm{~km} \mathrm{~s}^{-1}$. Although Peng et al. (2006) do not quote errors in their line-width measurements, the disagreement ( $\sim 5 \AA$ in the observed frame) is likely within their uncertainties.

Line widths of the $\mathrm{H} \beta$ and $\mathrm{H} \alpha$ broad-emission lines are given in Table 3 , while the continuum and broad-line spectral wavelength regions used are given in Table 4 . We measured them from the near-IR spectra following a similar procedure to the $\mathrm{C}$ IV line widths except that (1) the best GH polynomial fit was used for all line-width measurements, with the exception of $\mathrm{H} \alpha$ for SBS0909+532, because the S/N of the near-IR data was typically too poor to justify measurement directly from the data, (2) blended emission-line components were removed from each spectrum before the line width was measured, as described in the Appendix, and (3) a power-law, instead of a linear, continuum was fit to the $\mathrm{H} \beta$ spectrum of HS0810+2554 because it was fit simultaneously with additional blended emission-line components over a larger wavelength range.

For the objects where we lack the near-IR spectroscopic observations, we rely on the published $\mathrm{H} \alpha$ and $\mathrm{H} \beta$ line widths of GPL10. These measurements were done using methods somewhat different from ours. While we consider most of the GPL10 FWHM estimates to be reliable, there are some that we believe are suspect because (1) they were measured from very 


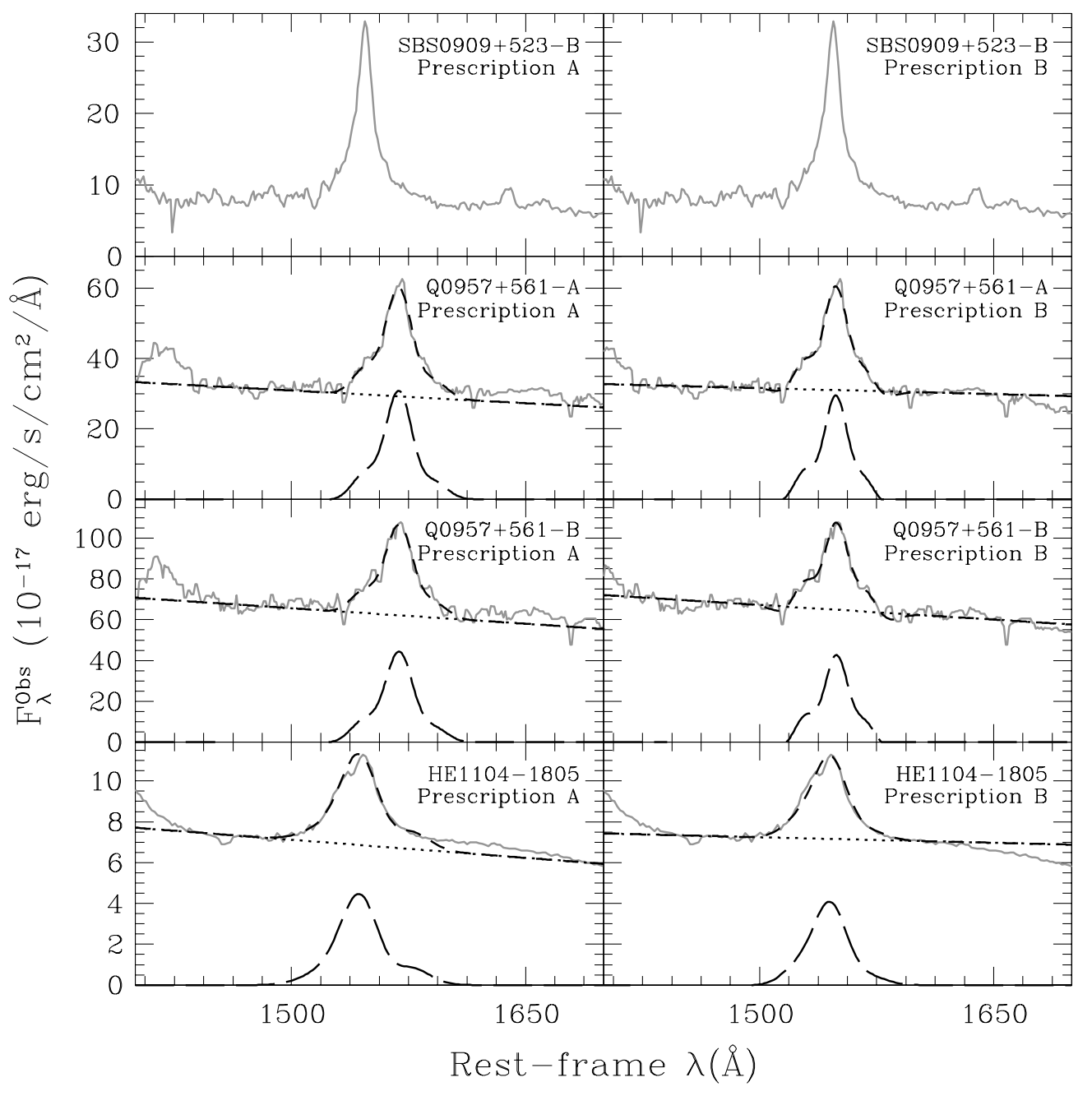

Figure 5. (Continued)

low S/N spectra, (2) the lines were not fully contained in the wavelength range of the spectrum, and/or (3) we do not agree with the narrow-line component models subtracted before the line width was measured. In the relevant figures and tables, we differentiate between the Balmer line velocity widths we think are reliable (group I, solid symbols) and those we believe are affected by any of these issues (group II, open symbols). Individual objects can be in both groups because these issues may affect only one of the Balmer lines. We also include in group I the $\mathrm{H} \alpha$ and $\mathrm{H} \beta$ line-width measurements from our new IR spectra. The decision to split our sample is a conservative choice, and our conclusions are not significantly modified when the group II line widths are included.

\subsection{Line-width Measurement Uncertainties}

Line-width measurements can be affected by sources of error that are difficult to model, as they depend not only on the overall $\mathrm{S} / \mathrm{N}$ ratio, but also on the line profile and the presence of sky emission and absorption lines, with the latter being of particular importance in the near-IR. We use a Monte Carlo approach to determine the uncertainties in our line-width measurements. Using the flux uncertainty per pixel in each spectrum and the best-fit GH line profile (with the exception of the optical SDSS1138+0314 and SBS0909+532 spectra; see the Appendix), we produced 1000 resampled spectra by adding random Gaussian deviates based on the error spectrum to the flux in each pixel of the GH model spectrum and then remeasured the line width using the methods described in the previous section. For the UV/optical spectra from the literature without an error spectrum, we estimated one by propagating the measured $\mathrm{S} / \mathrm{N}$ of a small continuum window near the $\mathrm{C}$ IV $\lambda 1549$ emission line to the overall spectrum. In this case, $\delta F_{\lambda}$, the flux error in a pixel of wavelength $\lambda$ with flux $F_{\lambda}$, is given by

$$
\delta F_{\lambda}=\sqrt{\frac{\lambda_{c}}{\lambda} F_{\lambda_{c}} F_{\lambda}}\left(\frac{\mathrm{S}}{\mathrm{N}}\right)_{\mathrm{c}}^{-1},
$$

where $\lambda_{c}$ and $F_{\lambda_{c}}$ are the average wavelength and flux per unit wavelength of the continuum window chosen, and $(\mathrm{S} / \mathrm{N})_{\mathrm{c}}$ is the signal-to-noise ratio per pixel in the chosen continuum window. This equation is constructed by assuming that the only source of error is Poisson fluctuations, and that the number of detected photons is proportional to $F_{\lambda}(h c / \lambda)^{-1}$, where the proportionality constant is empirically determined in the continuum window from $(\mathrm{S} / \mathrm{N})_{c}, \lambda_{c}$, and $F_{\lambda_{c}}$. This approach neglects the sky background and the presence of strong absorption or emission sky lines, which is reasonable for the UV/optical spectra. It also neglects changes in the instrument sensitivity as a function of wavelength and assumes a constant pixel wavelength width, both of which are reasonable because the continuum $\mathrm{S} / \mathrm{N}$ is measured in close proximity to the emission line of interest. While the parametric fits are not 


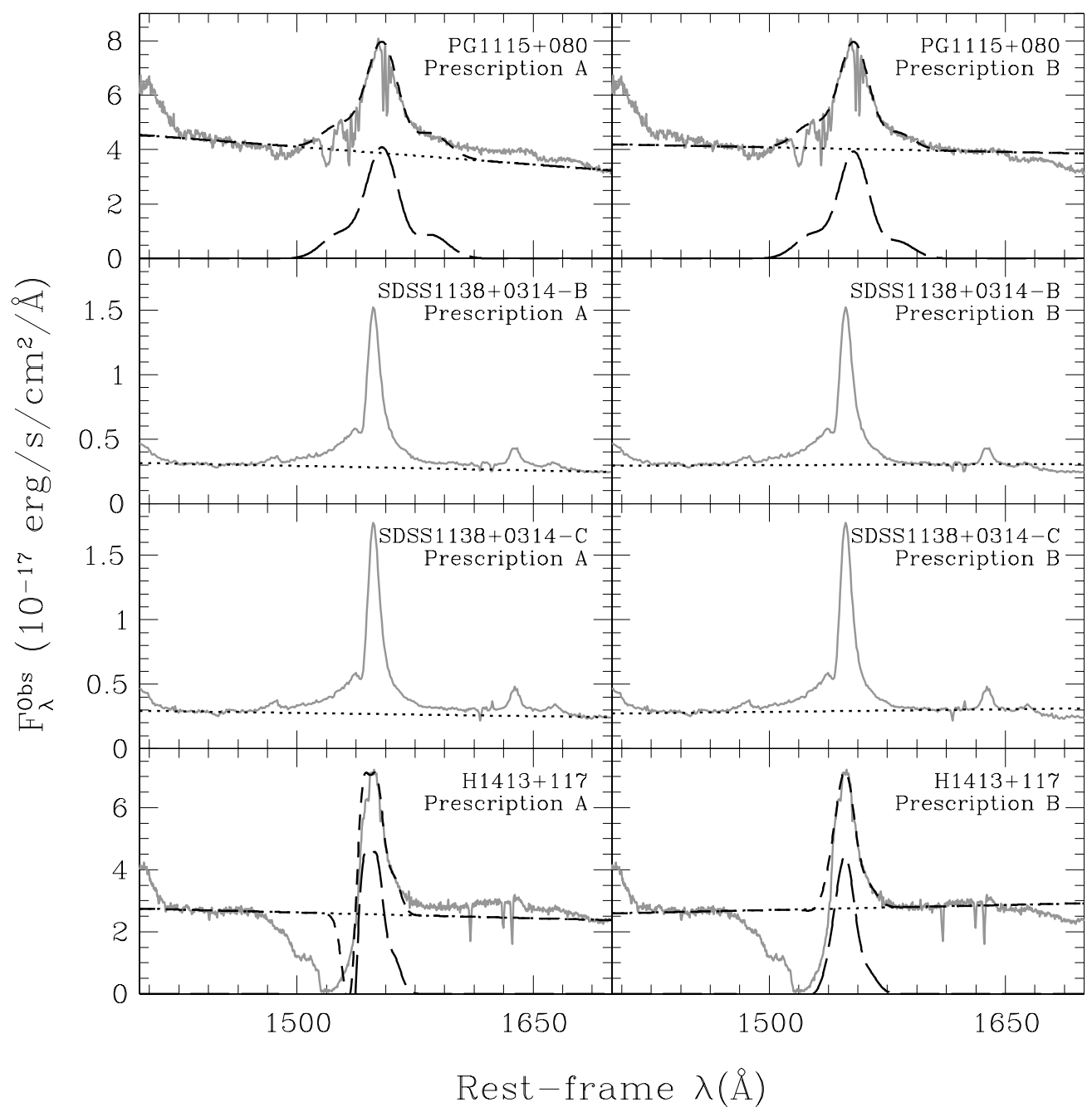

Figure 5. (Continued)

exact representations of each line, this still provides a reasonable estimate of the fractional uncertainties.

\subsection{Luminosity Measurements}

We estimated the continuum luminosities at $5100 \AA$ by fitting the AGN SED template of Assef et al. (2010) to the unmagnified quasar magnitudes obtained from the CASTLES project HST NICMOS imaging. To correct the observed quasar fluxes for the lens magnification, we modeled each system using the astrometry and lens galaxy photometry from the CASTLES HST WFPC2 and NICMOS observations following the procedures of Lehár et al. (2000). The image is decomposed into a set of point sources for the quasars, de Vaucouleurs models for the lens galaxy and, if necessary, a lensed host component, convolved with model or empirical point-spread functions (PSFs). The resulting component positions and image fluxes were modeled using lensmodel (Keeton 2001). The lens was modeled as a singular isothermal ellipsoid in an external shear with the ellipsoid's orientation and ellipticity constrained by those of the light of the lens galaxy and a weak prior on the external shear. The models were not tightly constrained to match the observed fluxes due to systematic errors in image flux ratios such as source variability and microlensing. Aside from substructure, the dominant uncertainty in the magnifications is the radial mass distribution of the lens (see Kochanek et al. 2004), and this is less than a factor of two even if we allow the full range of models between a flat rotation curve and a constant $M / L$ model. Since we have extensive evidence that lenses have mass distributions corresponding to flat rotation curves on these scales (e.g., Rusin et al. 2003; Jiang \& Kochanek 2007; Koopmans et al. 2009), the model uncertainties are considerably less than this factor and the uncertainties are dominated by the systematic uncertainties in the image fluxes. Table 1 lists the magnifications used for each object in the sample. The only object for which a different model was used is Q0957+561, where we used the magnifications determined by Fadely et al. (2010).

We did not apply reddening corrections other than removing Galactic foreground extinction (see below), as the requirement that $\mathrm{CIV}$ is observable in the $\mathrm{UV} /$ optical severely limits the presence of dust absorption, especially at rest-frame $5100 \AA$. For all four-image lenses, we estimated the true source flux for all images, rejected the highest and lowest estimates, and averaged the remaining two to limit the effects of microlensing. For two-image lenses we simply averaged the two estimates. Table 1 shows the estimated unmagnified $H$-band magnitude of each quasar. Note that in general we did not apply a correction for variability. Although there is a 5-10 year time difference between the CASTLES and the GPL10 Triplespec observations, the typical uncertainty introduced falls well below the systematic uncertainties in the SE BH mass estimates. An 


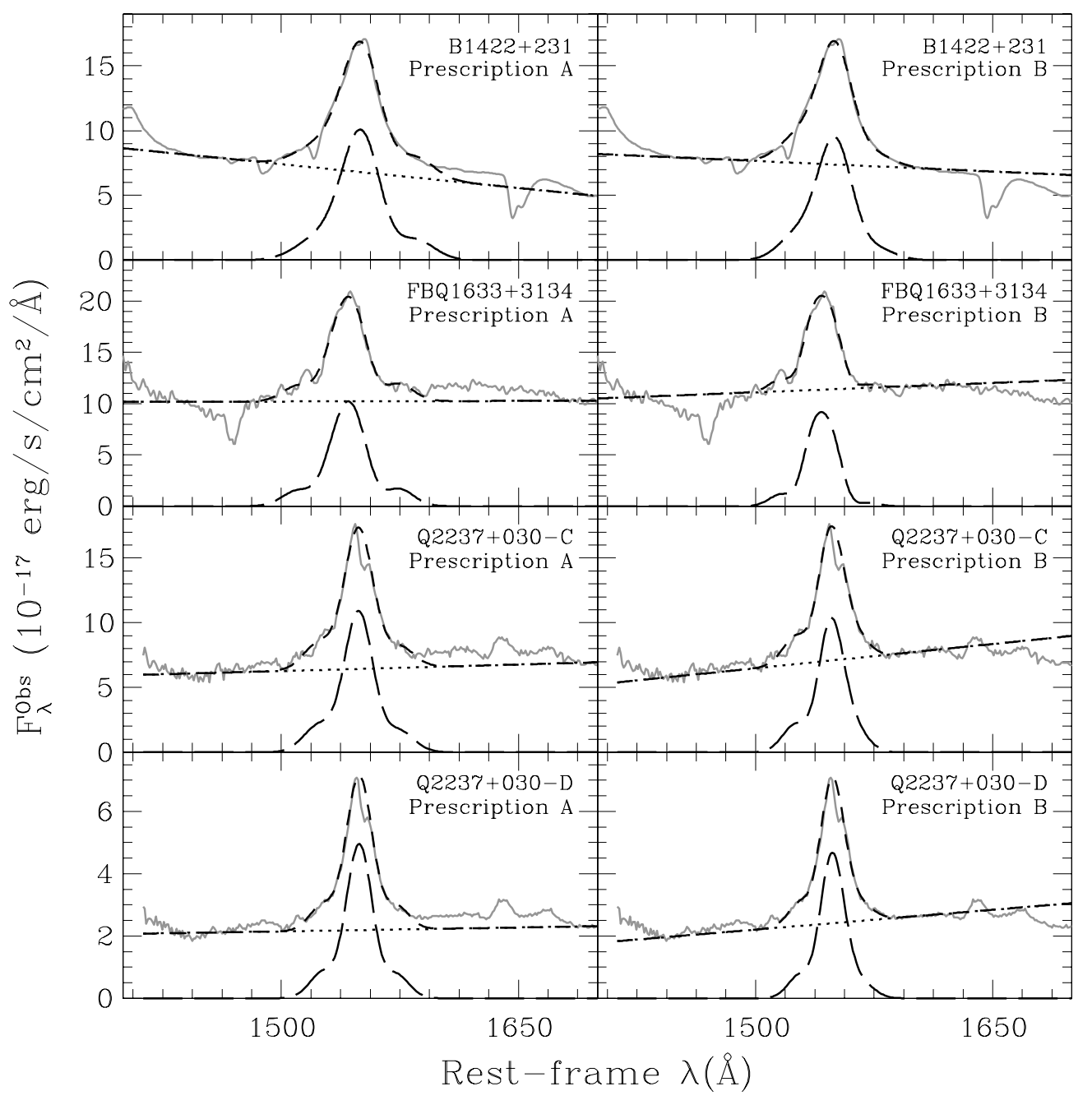

Figure 5. (Continued)

estimate of the typical variability of a quasar can be obtained from measurements of their structure function. Using the powerlaw fit of Vanden Berk et al. (2004) to the $i$-band structure function of SDSS quasars, we find that the typical quasar would experience a change in magnitude of approximately 0.2 mag for a rest-frame time lag of 1500 days (approximately 10 years in the observer's frame for our lowest redshift quasar). A change of 0.2 mag results in a change to the $\mathrm{BH}$ mass estimate of 0.04 dex, well below their typical error bar of $0.3 \mathrm{dex}$, and we would expect the $H$-band variability to be still smaller, as the average variability amplitude decreases with increasing wavelength (see, e.g., Vanden Berk et al. 2004; MacLeod et al. 2010).

For SDSS1138+0314, HS0810+2554, and SBS0909+532, we performed an absolute flux calibration of the near-IR spectra and measured the $5100 \AA$ continuum luminosity directly. The calibration for the first object is discussed in detail in Section 2.1. For HS0810+2554 we fit a power law to the continuum of our MDM CCDS spectrum (see Section 2.2) and extrapolated it to rest-frame $5100 \AA$. For SBS0909+532 we calibrated the spectrum using the HST NICMOS $H$-band photometry, as the object did not show significant flux variations between the two relevant epochs (J. Muñoz 2010, private communication).

To obtain the rest-frame continuum UV luminosities at $1350 \AA$ and $1450 \AA$, we flux calibrated the spectra whenever it was necessary and measured the flux by fitting a straight line to the region between rest-frame $1349 \AA$ and $1355 \AA$ for the estimate at $1350 \AA$ and to the region between $1440 \AA$ and $1460 \AA$ for the estimate at $1450 \AA$. We corrected these luminosities for foreground Galactic extinctions obtained through the NASA/IPAC Extragalactic Database ${ }^{17}$ from the dust maps of Schlegel et al. (1998). Errors in the continuum luminosity will be dominated by the uncertainties in the magnification models, which are hard to quantify. We assume a conservative error of $20 \%$ in each continuum luminosity estimate.

GPL10 obtained continuum luminosities at $5100 \AA$ for their sample of objects by following a similar approach. They fit a power law to the unmagnified $H S T$ photometry from the CASTLES survey, using the lensing models of Peng et al. (2006). In comparison to GPL10 we observe that our luminosity estimates are, on average, $0.20 \pm 0.05$ dex smaller. The offset is likely caused by a combination of the differences in the lensing models, in the prescription used to deal with the flux ratio anomalies, and in the use of the AGN SED template of Assef et al. (2010) instead of the power-law fits of Peng et al. (2006). We note that this offset translates to $0.1 \mathrm{dex}$ in $\mathrm{BH}$ mass, well below the uncertainties we estimate for our SE mass measurements in the next section. We also note that our conclusions are unaltered if we replace our $5100 \AA$ continuum luminosity estimates with those of GPL10 for all objects where this is possible.

\footnotetext{
17 http://nedwww.ipac.caltech.edu/
} 
Table 3

Velocity Widths

\begin{tabular}{|c|c|c|c|c|c|c|c|c|}
\hline \multirow[t]{2}{*}{ Object } & \multicolumn{8}{|c|}{ Broad Emission Line Velocity Widths $/ 10^{3} \mathrm{~km} \mathrm{~s}^{-1}$} \\
\hline & $\mathrm{FWHM}_{\mathrm{C}_{\text {IV }}}-\mathrm{p} . \mathrm{A}$ & FWHM $_{\mathrm{C}_{\text {IV }}}-$ p.B & $\sigma_{l, \mathrm{C} \text { IV }}-\mathrm{p} . \mathrm{A}$ & $\sigma_{l, \mathrm{C} \text { IV }}-\mathrm{p} . \mathrm{B}$ & $\mathrm{FWHM}_{\mathrm{H} \beta}{ }^{\mathrm{a}}$ & $\sigma_{l, \mathrm{H} \beta}$ & $\mathrm{FWHM}_{\mathrm{H} \alpha}{ }^{\mathrm{a}}$ & $\sigma_{l, \mathrm{H} \alpha}$ \\
\hline Q0142-100 & $5.20 \pm 0.18$ & $4.75 \pm 0.22$ & $3.67 \pm 0.04$ & $3.67 \pm 0.07$ & $2.70 \pm 0.60^{b}$ & $\ldots$ & $3.80 \pm 0.30^{\mathrm{b}}$ & $\ldots$ \\
\hline SDSS0246-0825 & $4.43 \pm 0.24$ & $4.40 \pm 0.22$ & $3.09 \pm 0.06$ & $2.51 \pm 0.04$ & $2.50 \pm 0.60$ & $\ldots$ & $2.50 \pm 0.20$ & $\ldots$ \\
\hline HS0810+2554 & $3.68 \pm 0.14$ & $3.53 \pm 0.16$ & $3.40 \pm 0.05$ & $2.85 \pm 0.08$ & $4.40 \pm 0.06$ & $2.21 \pm 0.04$ & $3.80 \pm 0.00$ & $\ldots$ \\
\hline SBS0909+532 & $2.38 \pm 0.08$ & $2.36 \pm 0.08$ & $2.90 \pm 0.04$ & $2.82 \pm 0.05$ & $3.95 \pm 0.17$ & $2.20 \pm 0.05$ & $3.06 \pm 0.34$ & $5.24 \pm 0.04$ \\
\hline Q0957+561 & $3.68 \pm 0.25$ & $3.47 \pm 0.08$ & $3.27 \pm 0.09$ & $2.272 \pm 0.007$ & $3.30 \pm 0.90$ & $\ldots$ & $3.00 \pm 0.20$ & $\ldots$ \\
\hline HE1104-1805 & $6.08 \pm 0.35$ & $5.75 \pm 0.05$ & $3.84 \pm 0.10$ & $2.897 \pm 0.004$ & $3.80 \pm 0.90$ & $\ldots$ & $4.70 \pm 0.20^{\mathrm{b}}$ & $\ldots$ \\
\hline PG1115+080 & $4.98 \pm 0.18$ & $4.67 \pm 0.13$ & $3.68 \pm 0.04$ & $3.40 \pm 0.04$ & $4.40 \pm 0.20$ & $\ldots$ & $4.00 \pm 0.10$ & $\ldots$ \\
\hline SDSS1138+0314 & $>2.02 \pm 0.15$ & $>1.99 \pm 0.18$ & $>3.12 \pm 0.04$ & $>2.40 \pm 0.11$ & $3.93 \pm 0.30$ & $2.08 \pm 0.18$ & $2.57 \pm 0.04$ & $1.90 \pm 0.05$ \\
\hline $\mathrm{H} 1413+117$ & $>2.62 \pm 0.95$ & $>2.54 \pm 0.37$ & $>3.78 \pm 0.15$ & $>1.82 \pm 0.07$ & $6.70 \pm 1.90$ & $\ldots$ & $5.30 \pm 0.80$ & $\ldots$ \\
\hline $\mathrm{B} 1422+231$ & $5.81 \pm 0.16$ & $5.56 \pm 0.02$ & $3.70 \pm 0.03$ & $3.321 \pm 0.006$ & $6.10 \pm 2.20^{b}$ & $\cdots$ & $\ldots$ & $\cdots$ \\
\hline FBQ1633+3134 & $>4.71 \pm 0.18$ & $>4.40 \pm 0.16$ & $>3.83 \pm 0.06$ & $>2.20 \pm 0.06$ & $4.60 \pm 0.90^{b}$ & $\ldots$ & $4.10 \pm 0.70$ & $\ldots$ \\
\hline $\mathrm{Q} 2237+030$ & $3.96 \pm 0.18$ & $3.78 \pm 0.12$ & $3.49 \pm 0.07$ & $2.51 \pm 0.05$ & $3.80 \pm 1.40$ & $\cdots$ & $4.80 \pm 0.60^{\mathrm{b}}$ & $\cdots$ \\
\hline
\end{tabular}

Notes.

${ }^{a}$ All $\mathrm{H} \alpha$ and $\mathrm{H} \beta$ line-width measurements correspond to those in Table 1 of GPL10, except for SDSS1138+0314, SBS0909+523, and the H $\beta$ widths of HS0810+2554.

b Group II line widths. See Section 3.1 for details.

Table 4

NIR Emission Line and Continuum Region Boundaries

\begin{tabular}{|c|c|c|c|c|c|c|}
\hline Object & $\begin{array}{c}\text { Emission } \\
\text { Line }\end{array}$ & $\mathrm{S} / \mathrm{N}^{\mathrm{a}}$ & $\begin{array}{c}\text { Blue Cont. } \\
(\AA)\end{array}$ & $\begin{array}{l}\text { Red Cont. } \\
(\AA)\end{array}$ & $\begin{array}{c}\text { Broad Line } \\
(\AA)\end{array}$ & $\begin{array}{l}\text { Res } \\
(\AA) \\
\end{array}$ \\
\hline $\mathrm{HS} 0810+2554^{\mathrm{a}}$ & $\mathrm{H} \beta$ & 36 & $4680-4710$ & $5080-5120$ & $4710-4960$ & 8.0 \\
\hline SBS0909+532-A & $\mathrm{H} \beta$ & 68 & $11205-11310$ & $11840-11855$ & $11380-11840$ & $\ldots$ \\
\hline SBS0909+532-B & $\mathrm{H} \beta$ & 22 & $11205-11310$ & $11840-11855$ & $11380-11840$ & $\cdots$ \\
\hline SBS0909+532-A & $\mathrm{H} \alpha$ & 25 & $14210-14440$ & $17500-17700$ & $14865-16650$ & $\ldots$ \\
\hline SBS0909+532-B & $\mathrm{H} \alpha$ & 18 & $14210-14440$ & $17500-17700$ & $14865-16650$ & $\ldots$ \\
\hline SDSS1138+0314 & $\mathrm{H} \beta$ & 12 & $16150-16300$ & $17485-17623$ & $16300-17100$ & 8.0 \\
\hline SDSS1138+0314 & $\mathrm{H} \alpha$ & 8 & $21780-21850$ & $23270-23310$ & $22135-23040$ & 8.0 \\
\hline
\end{tabular}

Note. ${ }^{\text {a }}$ Rest frame wavelengths are used here because line boundaries were chosen after the deblending procedure that transfers the spectrum into the rest frame.

\subsection{Black Hole Mass Estimates}

The width of a given broad emission line in a Type 1 AGN is primarily caused by the gravitational attraction of the supermassive $\mathrm{BH}$ on the gas in the BLR. Hence, the mass of the black hole, $M_{\mathrm{BH}}$, can be estimated from virial assumptions by

$$
M_{\mathrm{BH}}=f \frac{R_{\mathrm{BLR}}(\Delta v)^{2}}{G},
$$

where $\Delta v$ is the velocity dispersion of the BLR gas, estimated from the width of the broad emission line, $G$ is the gravitational constant, and $R_{\mathrm{BLR}}$ is the distance from the $\mathrm{BH}$ to the BLR. The factor $f$ is a scale factor of order unity that depends on the structure, kinematics, and inclination of the BLR (see, e.g., Collin et al. 2006 and references therein). The term $R_{\mathrm{BLR}}(\Delta v)^{2} / G$ is usually referred to as the virial product (VP) and encapsulates all the observable quantities for a single object. The radius of the BLR can only be measured through RM (see, e.g., Peterson et al. 2004), but has been shown to correlate well with the continuum luminosity (see, e.g., Kaspi et al. 2005; Bentz et al. 2006, 2009; Zu et al. 2011).

For the broad hydrogen emission lines we estimate the BLR radius using the $R_{\mathrm{BLR}}-\lambda L_{\lambda}(5100 \AA)$ relation of Bentz et al. (2009), which was calibrated using a large sample of RM AGNs. The $f$ factor of Equation (2) depends on the characterization of the line width, generally either the FWHM or the line dispersion, $\sigma_{l}$, as well as on the emission line being used. For estimating
$M_{\mathrm{BH}}$ from the width of the $\mathrm{H} \beta$ broad line, we use the $f$ factor calibrations of Collin et al. (2006) for the FWHM and for $\sigma_{l}$. While for $\sigma_{l}$ a unique $f$ factor of 3.85 for all AGNs suffices, Collin et al. (2006) argued that $f$ is strongly dependent on the line profile shape for FWHM-based estimates, where the shape was quantified as the ratio between the FWHM and $\sigma_{l}$. We choose, however, to use the best-fit fixed $f$ factor of 1.17 for FWHM instead of the line-shape dependent calibrations because Denney et al. (2009) have shown that $\sigma_{l}$ is affected by blending with other emission lines, making the correlation found by Collin et al. (2006) hard to interpret. For $\mathrm{H} \alpha$ there is no equivalent calibration of the $f$ factor, so we cannot directly estimate the $\mathrm{BH}$ masses. Instead, we use the relation determined by Greene \& Ho (2005) between the FWHM of $\mathrm{H} \alpha$ and $\mathrm{H} \beta$,

$$
\begin{aligned}
\text { FWHM }_{\mathrm{H} \beta}= & (1.07 \pm 0.07) \\
& \times 10^{3}\left(\frac{\mathrm{FWHM}_{\mathrm{H} \alpha}}{10^{3} \mathrm{~km} \mathrm{~s}^{-1}}\right)^{(1.03 \pm 0.03)} \mathrm{km} \mathrm{s}^{-1},
\end{aligned}
$$

to estimate the $\mathrm{H} \beta$ FWHM and then estimate $M_{\mathrm{BH}}(\mathrm{H} \alpha)$ using the same $f$ factor and $R_{\mathrm{BLR}}-L$ relation as for $M_{\mathrm{BH}}(\mathrm{H} \beta)$. Unfortunately, there is no equivalent transformation for $\sigma_{l}$, so we cannot use this measurement to estimate the mass of the $\mathrm{BH}$ from $\mathrm{H} \alpha$. 
Table 5

$\mathrm{H} \beta, \mathrm{H} \alpha$, and $\mathrm{C}$ IV BH Mass Estimates

\begin{tabular}{|c|c|c|c|c|c|c|c|}
\hline \multirow[t]{2}{*}{ Object } & \multicolumn{7}{|c|}{$\log M_{\mathrm{BH}} / M_{\odot}$} \\
\hline & FWHM $_{\mathrm{C}_{\text {IV }}}-$ p.A & $\mathrm{FWHM}_{\mathrm{C}_{\text {IV }}}-$ p.B & $\sigma_{l, \mathrm{C} \text { IV }}-$ p.A & $\sigma_{l, \mathrm{C} \text { Iv }}-$ p.B & $\mathrm{FWHM}_{\mathrm{H} \beta}$ & $\sigma_{l, \mathrm{H} \beta}$ & $\mathrm{FWHM}_{\mathrm{H} \alpha}$ \\
\hline Q0142-100 & $9.59 \pm 0.32$ & $9.51 \pm 0.33$ & $9.36 \pm 0.29$ & $9.36 \pm 0.29$ & $8.94 \pm 0.30^{\mathrm{a}}$ & . & $9.33 \pm 0.23^{\mathrm{a}}$ \\
\hline SDSS0246-0825 & $8.24 \pm 0.33$ & $8.23 \pm 0.33$ & $7.99 \pm 0.29$ & $7.81 \pm 0.29$ & $8.00 \pm 0.31$ & $\ldots$ & $8.08 \pm 0.23$ \\
\hline HS0810+2554 & $8.03 \pm 0.32$ & $7.99 \pm 0.33$ & $8.02 \pm 0.29$ & $7.87 \pm 0.29$ & $8.62 \pm 0.22$ & $8.54 \pm 0.17$ & $8.65 \pm 0.22$ \\
\hline SBS0909+532 & $8.51 \pm 0.32$ & $8.51 \pm 0.32$ & $8.76 \pm 0.29$ & $8.73 \pm 0.29$ & $9.29 \pm 0.23$ & $9.29 \pm 0.17$ & $9.15 \pm 0.24$ \\
\hline Q0957+561 & $9.02 \pm 0.33$ & $8.97 \pm 0.32$ & $8.98 \pm 0.29$ & $8.67 \pm 0.29$ & $8.86 \pm 0.33$ & $\ldots$ & $8.87 \pm 0.23$ \\
\hline HE1104-1805 & $9.37 \pm 0.33$ & $9.32 \pm 0.32$ & $9.04 \pm 0.29$ & $8.79 \pm 0.29$ & $8.77 \pm 0.30$ & $\ldots$ & $9.05 \pm 0.23^{\mathrm{a}}$ \\
\hline PG1115+080 & $8.83 \pm 0.32$ & $8.78 \pm 0.32$ & $8.64 \pm 0.29$ & $8.57 \pm 0.29$ & $8.66 \pm 0.23$ & $\ldots$ & $8.68 \pm 0.22$ \\
\hline SDSS1138+0314 & $7.71 \pm 0.33^{b}$ & $7.69 \pm 0.33^{b}$ & $8.15 \pm 0.29^{b}$ & $7.93 \pm 0.29^{b}$ & $8.50 \pm 0.23$ & $8.47 \pm 0.19$ & $8.22 \pm 0.22$ \\
\hline H1413+117 & $8.41 \pm 0.45^{\mathrm{b}}$ & $8.39 \pm 0.35^{b}$ & $8.80 \pm 0.29^{b}$ & $8.17 \pm 0.29^{\mathrm{b}}$ & $9.39 \pm 0.33$ & $\ldots$ & $9.29 \pm 0.26$ \\
\hline B $1422+231$ & $9.69 \pm 0.32$ & $9.65 \pm 0.32$ & $9.37 \pm 0.29$ & $9.27 \pm 0.29$ & $9.72 \pm 0.38^{\mathrm{a}}$ & $\ldots$ & \\
\hline FBQ1633+3134 & $8.88 \pm 0.32^{\mathrm{b}}$ & $8.82 \pm 0.32^{\mathrm{b}}$ & $8.77 \pm 0.29^{b}$ & $8.29 \pm 0.29^{b}$ & $9.11 \pm 0.28^{\mathrm{a}}$ & $\ldots$ & $9.11 \pm 0.27$ \\
\hline $\mathrm{Q} 2237+030$ & $8.67 \pm 0.33$ & $8.63 \pm 0.32$ & $8.63 \pm 0.29$ & $8.34 \pm 0.29$ & $9.08 \pm 0.39$ & $\ldots$ & $9.38 \pm 0.25^{\mathrm{a}}$ \\
\hline
\end{tabular}

Notes. All BH masses correspond to those obtained from Equations (4), (5), and (6). None of the corrections discussed in Sections 4.1 and 4.2 have been applied.

${ }^{a}$ Based on group II line width. See Section 3.1 for details.

${ }^{\mathrm{b}}$ Should be considered as lower bound. See the Appendix for details.

Combining Equations (2) and (3) with the $R_{\mathrm{BLR}}-\lambda L_{\lambda}(5100 \AA)$ relation of Bentz et al. (2009) we get

$$
\begin{aligned}
M_{\mathrm{BH}}(\mathrm{H} \beta)= & 6.71 \times 10^{6} f\left(\frac{\Delta v_{\mathrm{H} \beta}}{10^{3} \mathrm{~km} \mathrm{~s}^{-1}}\right)^{2} \\
& \times\left(\frac{\lambda L_{\lambda}(5100 \AA)}{10^{44} \mathrm{erg} \mathrm{s}^{-1}}\right)^{0.52} M_{\odot} \\
M_{\mathrm{BH}}(\mathrm{H} \alpha)= & 7.68 \times 10^{6} f\left(\frac{\mathrm{FWHM}_{\mathrm{H} \alpha}}{10^{3} \mathrm{~km} \mathrm{~s}^{-1}}\right)^{2.06} \\
& \times\left(\frac{\lambda L_{\lambda}(5100 \AA)}{10^{44} \mathrm{erg} \mathrm{s}^{-1}}\right)^{0.52} M_{\odot},
\end{aligned}
$$

where in Equation (4) $\Delta v_{\mathrm{H} \beta}$ can be either the line dispersion or the FWHM. Because Equation (5) is fully dependent upon the scaling relations for $\mathrm{H} \beta$, the $f$ factor in it is the same as for $\mathrm{FWHM}_{\mathrm{H} \beta}$ in Equation (4). Table 5 shows our BH mass estimates based on $\mathrm{H} \alpha$ and $\mathrm{H} \beta$ for all objects in the sample.

For the UV/optical spectra we use the empirical $M_{\mathrm{BH}}$ calibrations of VP06 for the CIV broad emission line given by

$$
M_{\mathrm{BH}}(\mathrm{C} \mathrm{IV})=10^{\kappa}\left(\frac{\Delta v_{\mathrm{CIV}}}{10^{3} \mathrm{~km} \mathrm{~s}^{-1}}\right)^{2}\left(\frac{\lambda L_{\lambda}(1350 \AA)}{10^{44} \mathrm{erg} \mathrm{s}^{-1}}\right)^{0.53} M_{\odot},
$$

where $\Delta v$ is either FWHM or $\sigma_{l}$, and $\kappa=6.66 \pm 0.01$ or $6.73 \pm 0.01$, respectively, for these line-width characterizations. The constant $\kappa$ implicitly contains the $f$ factor, which is assumed to be a constant for all objects. Whenever possible, we use the observed $1350 \AA$ flux to determine the continuum luminosity. Unfortunately, $1350 \AA$ is not within the observed wavelength range of all the UV/optical spectra we use. In these cases we estimate the continuum luminosity at $1350 \AA$ using the observed flux at $1450 \AA$, as VP06 have shown $L_{\lambda}$ at these wavelengths to be equivalent. We list our $\mathrm{C}$ IV BH mass estimates in Table 5 for both prescriptions used to measure the widths of CIV. As expected, masses determined from the FWHM are highly consistent for both prescriptions, with a mean difference of
0.04 dex and a scatter of 0.02 dex, with the average prescription $\mathrm{B}$ based mass estimates being smaller. The agreement is much worse for $\sigma_{l}$, with a mean difference of $0.23 \mathrm{dex}$, in the sense that $\mathrm{B}$ is smaller, and a scatter of $0.18 \mathrm{dex}$.

We estimate the uncertainties in our $\mathrm{BH}$ mass estimates by propagating the errors in the velocity widths and in the continuum luminosities. For masses based on the width of the broad hydrogen lines, we also propagate the uncertainties in the $f$ factor and in $R_{\mathrm{BLR}}$. Collin et al. (2006) determined that the uncertainty in $f$ when using $\sigma_{l}$ is $30 \%$, while that in FWHM is $43 \%$. For $R_{\text {BLR }}$ we assume the intrinsic scatter of 0.11 dex estimated by Peterson (2010) for the radius-luminosity relation. Adding the uncertainties in $f$ and $R_{\mathrm{BLR}}$ is not possible for the C IV estimates of the $\mathrm{BH}$ masses. Instead, we add the measurement errors and the intrinsic scatter between $\mathrm{C}$ IV and $\mathrm{RM} \mathrm{BH}$ mass estimates in quadrature. Using the sample of VP06, we estimate intrinsic scatters of 0.32 dex and 0.28 dex for FWHM and $\sigma_{l}$, respectively. VP06 found that the total scatter, including measurement errors, was 0.32 dex for both linewidth characterizations of $\mathrm{C}$ IV, showing that the intrinsic scatter dominates over measurement errors, especially for FWHM estimates.

\section{BIASES IN CIV BLACK HOLE MASS ESTIMATES}

In this section, we use the sample described in Section 2 to study biases in the C IV BH mass estimates. We first compare how the mass depends on the characterization of the C IV line width, and then we proceed to compare these rest-frame UV estimates to those based on the $\mathrm{H} \alpha$ and $\mathrm{H} \beta$ emission lines. In the next section, we will compare our results with those of other studies on the relations between $\mathrm{C}$ IV and $\mathrm{H} \beta$ BH estimated masses.

\subsection{Comparison of FWHM and $\sigma_{l}$ Derived Masses}

Given that we have measured both FWHM and $\sigma_{l}$ for C IV in all our objects, the simplest test we can perform is to determine if there are any biases between them as BH mass estimators. Both measurements have advantages, and some contention exists in the literature as to which constitutes a more reliable mass estimator (see Peterson et al. 2004 and references therein). 


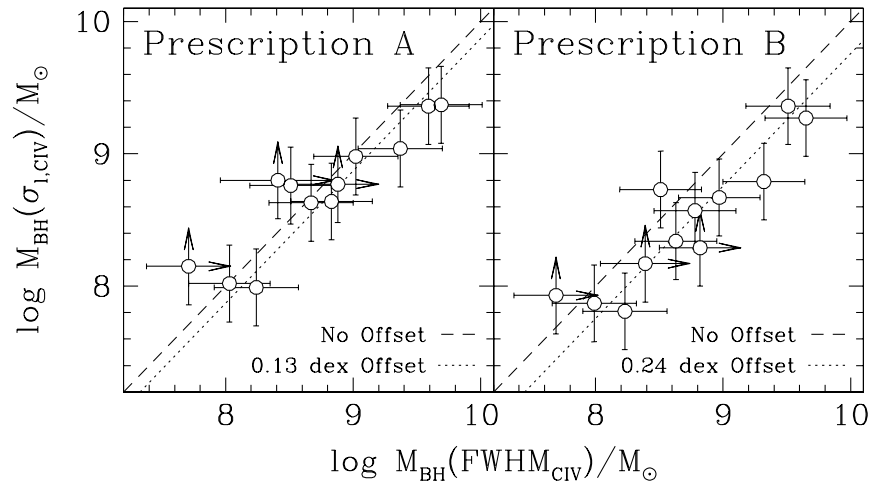

Figure 6. Comparison of $\mathrm{C}$ IV $\mathrm{BH}$ masses derived from the FWHM and $\sigma_{l}$ velocity widths for all objects in our sample using the relations of VP06. The left (right) panel compares the $\mathrm{BH}$ mass estimates based on the prescription A (B) line-width measurements of C IV. Masses are equal along the dashed line and the dotted line correspond to the best-fit offset. The objects with arrows correspond to those for which we believe our C IV line-width measurements to be lower bounds.

Figure 6 compares the $\mathrm{CIV}$-based $\mathrm{BH}$ masses determined for both line-width estimates and for the two continuum and line blending prescriptions $\mathrm{A}$ and $\mathrm{B}$, respectively. A clear bias is observed for both prescriptions, where most objects have a lower estimated BH mass if we use $\sigma_{l}$ instead of the FWHM. The bias for prescription A (B) width measurements seems to be well represented by a constant offset of $K=0.13 \pm 0.06$ dex $(0.24 \pm 0.07 \mathrm{dex})$ or, equivalently, a factor of $1.3(1.7)$. We fit for $K$ while simultaneously fitting for the intrinsic scatter between the two mass estimators by adding a scatter $S$ in quadrature to the error of each logarithmic mass difference. Note that the logarithmic mass difference does not depend on the continuum luminosity or the intrinsic scatter with respect to the RM estimates. In practice we maximize the likelihood

$$
\mathcal{L}=\left(\left\langle\sigma^{2}\right\rangle+S^{2}\right)^{-1 / 2}\left[\prod_{i=1}^{N}\left(\sigma_{i}^{2}+S^{2}\right)^{-1 / 2}\right] e^{-\chi^{2}(S) / 2}
$$

where $\sigma_{i}^{2}$ is the variance due to measurement errors in the logarithmic mass difference of object $i$ and $\left\langle\sigma^{2}\right\rangle$ is its average over all objects. We exclude objects for which we consider the $\mathrm{CIV}$-based $\mathrm{BH}$ mass estimates to be lower bounds due to absorption. The leading factor in Equation (7) is a logarithmic prior on the overall dispersion. The best-fit scatter is similar for both prescriptions, with case A line widths producing $S=0.16$ dex while case B ones have $S=0.19$ dex. Since the logarithmic mass difference only depends on the line widths and not on the continuum luminosities, the constant $\mathrm{BH}$ mass offsets $K$ can also be expressed as an offset between the linewidth characterizations. As such, these values imply an offset of $0.10 \pm 0.03$ dex $(0.16 \pm 0.04$ dex $)$ between the FWHM and $\sigma_{l}$ line-width characterizations of C IV for prescription A (B).

It is not surprising that prescription A provides a smaller offset between $\mathrm{BH}$ masses obtained from the FWHM and $\sigma_{l}$ of $\mathrm{C}_{\mathrm{IV}}$, as this prescription is modeled after that used by VP06, who used their measurements to determine Equation (6). However, given the similarity, the presence of a non-zero offset for prescription $\mathrm{A}$ is somewhat puzzling. If we examine the sample of VP06, the scatter is larger, $0.2 \mathrm{dex}$, and there is no offset $(-0.02 \pm 0.03 \mathrm{dex})$, although the lack of an offset is by definition small since both mass estimators were calibrated against the same RM data set.
The large overlap in the mass and continuum luminosity ranges of our sample and that of VP06 suggest that dependence on a secondary parameter is unlikely. Furthermore, we do not see any correlation of this bias with $\mathrm{BH}$ mass, continuum luminosity, or Eddington ratio. There is also no correlation with redshift, suggesting that it is unlikely to be an evolutionary trend. The only other major difference between the samples is lensing by foreground galaxies. This, however, is very unlikely to cause such an effect, as quasars are quite compact and strong lensing affects the whole object. Microlensing by the stars in the foreground galaxy could in principle distort the shape of the $\mathrm{C}$ IV broad emission lines due to the spatial dependence of their velocity structure, but this is very unlikely for two reasons. First, the width of C IV is typically well below $10,000 \mathrm{~km} \mathrm{~s}^{-1}$, constraining the location of the gas to a distance greater than $\gtrsim 10^{3}$ Schwarzschild radii $\left(R_{S}\right)$ from the $\mathrm{BH}$, while microlensing is only observed to have significant effects on scales below $100 R_{S}$ (Morgan et al. 2010). Second, the gas moving at the highest velocities is expected to be closest to the $\mathrm{BH}$, so microlensing would tend to magnify the wings of the line more than the core, and hence producing the inverse of the effect we see by making $\sigma_{l}$ too large rather than too small compared to the FWHM. While microlensing can also produce regions of demagnification in the source plane, these are of very large spatial extent, and so it is unlikely to see significant magnification variations across the BLR.

It is likely then that other minor differences in the method we use to measure $\sigma_{l}$ as compared to VP06 give rise to the remaining bias. Denney et al. (2009) showed that estimates of $\sigma_{l}$ depend on the exact prescription used for the line-width measurement and the segregation of blended emission for $\mathrm{H} \beta$. Our investigation shows that this may be the case for C IV as well (see also Fine et al. 2010). However, the remarkably low scatter in Figure 6 suggests that if $\sigma_{l}$ is measured in a self-consistent manner it can be as accurate as the FWHM for estimating BH masses, but the calibration will depend on the exact prescription. In the next section we will explore the reliability of the C IV FWHM and $\sigma_{l} \mathrm{BH}$ mass estimates by comparing them to those based on $\mathrm{H} \alpha$ and $\mathrm{H} \beta$.

\subsection{IV Compared to $H \alpha$ and $H \beta$}

Figures 7 and 8 compare the mass estimates based on the $\mathrm{H} \alpha$ and $\mathrm{H} \beta$ lines to those based on the width of C IV. We only show here (and for the rest of the figures) UV BH masses based on the prescription $\mathrm{B}$ width measurements of C IV. The FWHMbased $\mathrm{BH}$ masses are almost equal for prescriptions $\mathrm{A}$ and $\mathrm{B}$ (see Section 3.4), but they show a systematic offset for the $\sigma_{l}$ estimates (see Section 4.1). We adopt the prescription B masses for the rest of this section, but our conclusions are unaltered if we instead use prescription A measurements. We have made the assumption that the CIV FWHM mass estimates are unbiased, and so those obtained from the prescription $\mathrm{B} \sigma_{l}$ measurement of C IV have been shifted by the systematic offset of 0.24 dex derived in the previous section.

We measure no significant offset between the C IV-based and $\mathrm{H} \alpha$ - or $\mathrm{H} \beta$-based masses when using only objects with $\mathrm{C}$ IV line widths that are not lower bounds and have reliable Balmer line widths (group I). We find best-fit offsets of $-0.12 \pm 0.15$, $-0.11 \pm 0.16,-0.15 \pm 0.16$, and $-0.19 \pm 0.18$ dex for panels (a), (b), (c), and (d), respectively, of Figure 8, with residual scatter of $0.30,0.23,0.46$, and 0.38 dex. Including the objects with group II $\mathrm{H} \alpha$ and $\mathrm{H} \beta$ line-width estimates does not change this conclusion, with best-fit offsets of $-0.05 \pm 0.14,-0.13 \pm$ 


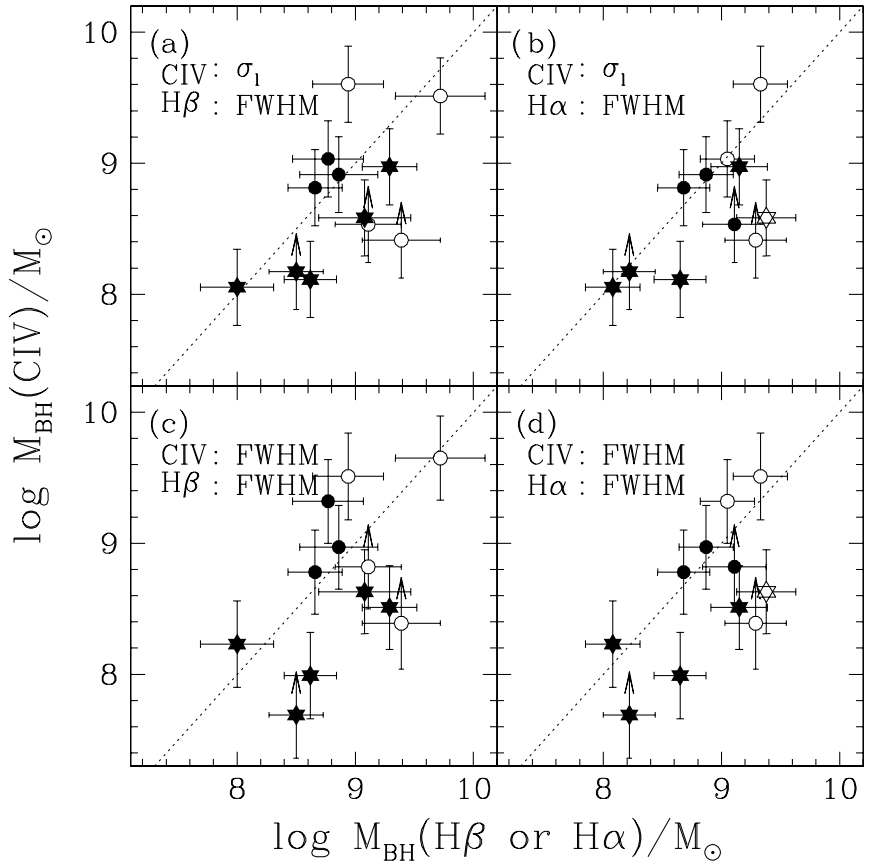

Figure 7. Comparison between $\mathrm{BH}$ masses estimated from the prescription $\mathrm{B}$ $\sigma_{l}$ and FWHM of C IV and from the FWHM of $\mathrm{H} \alpha$ and $\mathrm{H} \beta$. For the estimates based on the line dispersion of C IV we have added the systematic offset of 0.24 dex described in Section 4.1. Solid symbols correspond to the objects with group I $\mathrm{H} \alpha$ or $\mathrm{H} \beta$ line-width estimates, while open symbols correspond to those with group II estimates. Six-pointed stars mark the objects not considered in the analysis of GPL10 The dotted line shows where the BH masses are equal.

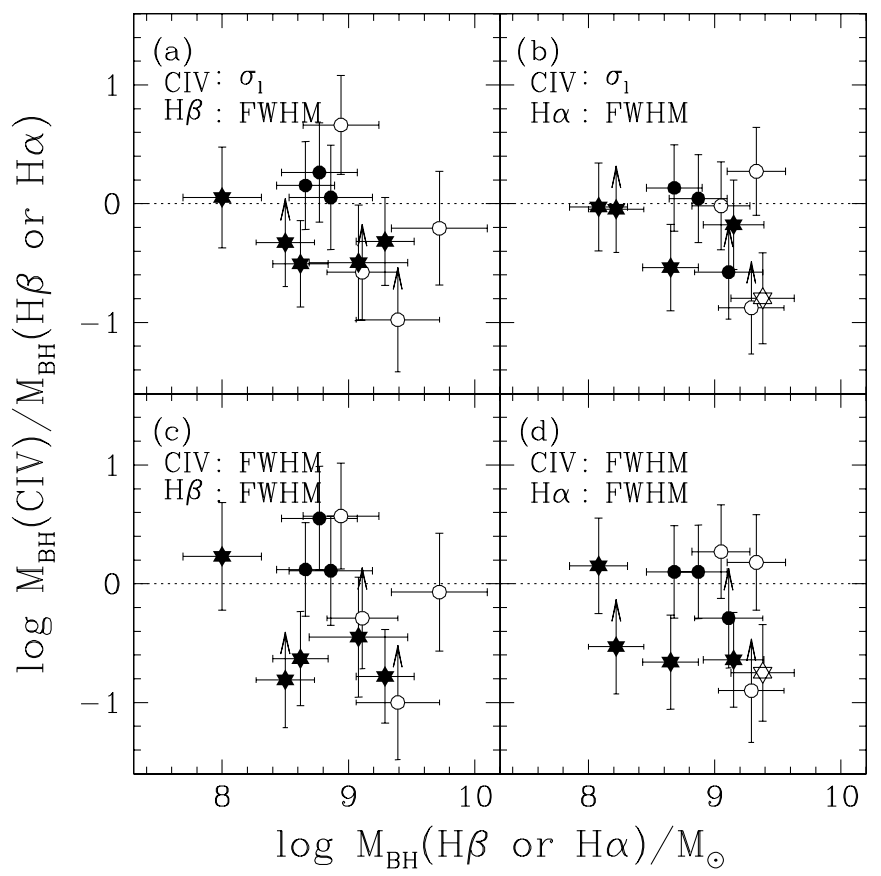

Figure 8. Ratio between the $\mathrm{C}$ IV and $\mathrm{H} \beta / \mathrm{H} \alpha$ mass estimates as a function of the corresponding hydrogen line mass estimate. Symbols and lines have the same definitions as in Figure 7.

$0.13,-0.07 \pm 0.15$, and $-0.15 \pm 0.14$ dex, respectively, with measured scatters of $0.36,0.33,0.46$, and 0.41 dex. The lack of offsets confirms our assumption that $\mathrm{C}$ IV FWHM BH masses are unbiased and that only those based on $\sigma_{l}$ need to be corrected. The constant offset fits yield $\chi^{2}$ per degree of freedom $\left(\chi_{v}^{2}\right)$ values of $0.6,0.5,1.4$, and 1.1 for panels (a), (b), (c), and (d)

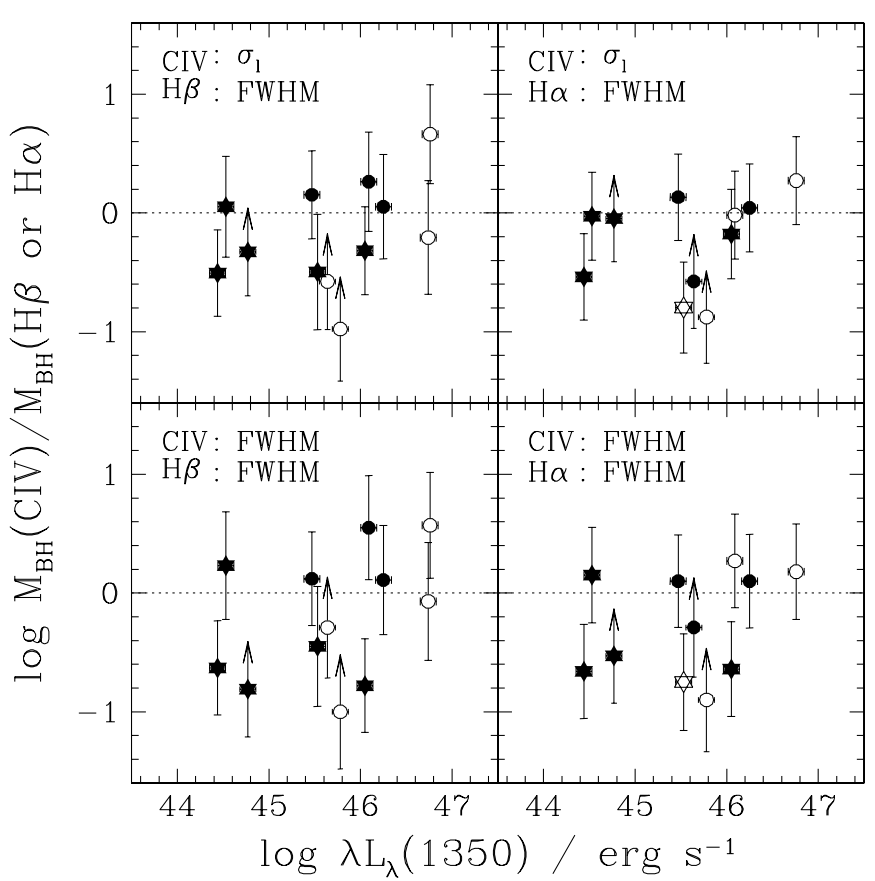

Figure 9. Same as Figure 8, but as a function of the UV continuum luminosity.

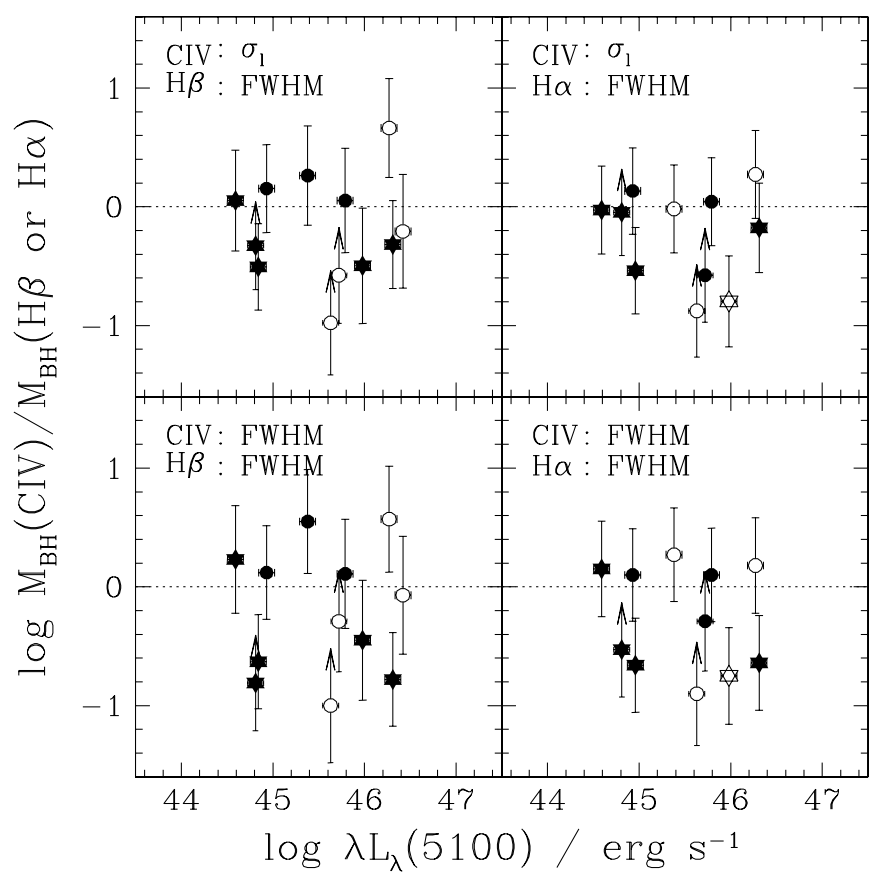

Figure 10. Same as Figure 8 , but as a function of the $5100 \AA$ continuum luminosity.

of Figure 7 when using only the solid symbols. The scatter in each panel of Figure 7 is largely consistent with the estimated uncertainties, although the errors in the $\mathrm{CIV} \sigma_{l}$ masses may be slightly overestimated. We find no evidence based on the $\chi^{2}$ statistic that a slope different from unity is required to describe the relation between the logarithms of the $\mathrm{BH}$ masses (Figure 7), independent of whether we include the group II Balmer linewidth measurements.

We next investigate if the residuals between the C IV and Balmer line masses are correlated with any other observables. Figures 9-15 show the residuals as a function of the $1350 \AA$ and $5100 \AA$ continuum luminosities, redshift, Eddington ratio, 
Table 6

Correlations of BH Mass Residuals

\begin{tabular}{|c|c|c|c|c|c|c|c|c|}
\hline \multirow[t]{2}{*}{ Variable } & \multirow{2}{*}{$\begin{array}{c}\text { C IV } \\
\text { Width }\end{array}$} & \multirow{2}{*}{$\begin{array}{c}\text { Balmer } \\
\text { Line }\end{array}$} & \multicolumn{3}{|c|}{ Group I Estimates } & \multicolumn{3}{|c|}{ Group I and II Estimates } \\
\hline & & & $r_{s}$ & $N$ & $P_{\text {ran }}$ & $r_{s}$ & $N$ & $P_{\text {ran }}$ \\
\hline \multirow{4}{*}{$M_{\mathrm{BH}}$ Balmer lines } & $\sigma_{l}$ & $\mathrm{H} \beta$ & -0.214 & 7 & 0.644 & -0.183 & 9 & 0.637 \\
\hline & & $\mathrm{H} \alpha$ & 0.100 & 5 & 0.873 & -0.048 & 8 & 0.911 \\
\hline & FWHM & $\mathrm{H} \beta$ & -0.500 & 7 & 0.253 & -0.333 & 9 & 0.381 \\
\hline & & $\mathrm{H} \alpha$ & -0.400 & 5 & 0.505 & -0.143 & 8 & 0.736 \\
\hline \multirow[t]{4}{*}{$\lambda L_{\lambda}(1350 \AA)$} & $\sigma_{l}$ & $\mathrm{H} \beta$ & 0.357 & 7 & 0.432 & 0.467 & 9 & 0.205 \\
\hline & & $\mathrm{H} \alpha$ & 0.500 & 5 & 0.391 & 0.571 & 8 & 0.139 \\
\hline & FWHM & $\mathrm{H} \beta$ & 0.143 & 7 & 0.760 & 0.367 & 9 & 0.332 \\
\hline & & $\mathrm{H} \alpha$ & 0.100 & 5 & 0.873 & 0.429 & 8 & 0.289 \\
\hline \multirow[t]{4}{*}{$\lambda L_{\lambda}(5100 \AA)$} & $\sigma_{l}$ & $\mathrm{H} \beta$ & -0.214 & 7 & 0.644 & -0.067 & 9 & 0.865 \\
\hline & & $\mathrm{H} \alpha$ & -0.300 & 5 & 0.624 & -0.048 & 8 & 0.911 \\
\hline & FWHM & $\mathrm{H} \beta$ & -0.500 & 7 & 0.253 & -0.233 & 9 & 0.546 \\
\hline & & $\mathrm{H} \alpha$ & -0.700 & 5 & 0.188 & -0.214 & 8 & 0.610 \\
\hline \multirow[t]{4}{*}{ Redshift } & $\sigma_{l}$ & $\mathrm{H} \beta$ & 0.607 & 7 & 0.148 & 0.517 & 9 & 0.154 \\
\hline & & $\mathrm{H} \alpha$ & 0.500 & 5 & 0.391 & 0.500 & 8 & 0.207 \\
\hline & FWHM & $\mathrm{H} \beta$ & 0.750 & 7 & 0.052 & 0.583 & 9 & 0.099 \\
\hline & & $\mathrm{H} \alpha$ & 0.600 & 5 & 0.285 & 0.619 & 8 & 0.102 \\
\hline \multirow[t]{4}{*}{$L / L_{\text {Edd }}$} & $\sigma_{l}$ & $\mathrm{H} \beta$ & -0.036 & 7 & 0.939 & 0.200 & 9 & 0.606 \\
\hline & & $\mathrm{H} \alpha$ & -0.300 & 5 & 0.624 & 0.024 & 8 & 0.955 \\
\hline & FWHM & $\mathrm{H} \beta$ & -0.286 & 7 & 0.534 & 0.067 & 9 & 0.865 \\
\hline & & $\mathrm{H} \alpha$ & -0.200 & 5 & 0.747 & -0.071 & 8 & 0.867 \\
\hline \multirow[t]{4}{*}{ C Iv Blueshift } & $\sigma_{l}$ & $\mathrm{H} \beta$ & 0.536 & 7 & 0.215 & 0.033 & 9 & 0.932 \\
\hline & & $\mathrm{H} \alpha$ & 0.100 & 5 & 0.873 & -0.214 & 8 & 0.610 \\
\hline & FWHM & $\mathrm{H} \beta$ & 0.679 & 7 & 0.094 & 0.133 & 9 & 0.732 \\
\hline & & $\mathrm{H} \alpha$ & 0.600 & 5 & 0.285 & 0.143 & 8 & 0.736 \\
\hline \multirow[t]{4}{*}{ C IV asymmetry } & $\sigma_{l}$ & $\mathrm{H} \beta$ & 0.429 & 7 & 0.337 & 0.117 & 9 & 0.765 \\
\hline & & $\mathrm{H} \alpha$ & 0.300 & 5 & 0.624 & -0.048 & 8 & 0.911 \\
\hline & FWHM & $\mathrm{H} \beta$ & -0.393 & 7 & 0.383 & -0.333 & 9 & 0.381 \\
\hline & & $\mathrm{H} \alpha$ & 0.300 & 5 & 0.624 & -0.476 & 8 & 0.233 \\
\hline \multirow[t]{4}{*}{$\lambda L_{\lambda}(1350 \AA) / \lambda L_{\lambda}(5100 \AA)$} & $\sigma_{l}$ & $\mathrm{H} \beta$ & 0.929 & 7 & 0.003 & 0.883 & 9 & 0.002 \\
\hline & & $\mathrm{H} \alpha$ & 1.000 & 5 & 0.000 & 0.809 & 8 & 0.015 \\
\hline & FWHM & $\mathrm{H} \beta$ & 0.750 & 7 & 0.052 & 0.767 & 9 & 0.016 \\
\hline & & $\mathrm{H} \alpha$ & 0.700 & 5 & 0.188 & 0.857 & 8 & 0.007 \\
\hline
\end{tabular}

Notes. The table shows the correlation strength of the BH mass residuals as a function of each different variable, quantified by the Spearman rank order coefficient, $r_{s}$. Results are shown for group I and the combination of groups I and II measurements. In each case, $N$ indicates the number of QSOs used to estimate the correlation strength and $P_{\text {ran }}$ indicates the probability of observing such a correlation by chance if the variables are uncorrelated.

blueshift of the C IV line, asymmetry of C IV (parameterized by the ratio of the widths red and blue of the centroid), and the ratio of the UV and optical continuum luminosities. Table 6 summarizes the significance of the correlations based on their Spearman rank-order coefficients. Only the correlation with the ratio of the rest-frame optical and UV continuum luminosities is significant (Figure 15). Figure 16 compares the $\mathrm{C}$ IV and Balmer line derived $\mathrm{BH}$ masses after rescaling the $\mathrm{C}$ IV masses using the best-fit correlation determined from the corresponding panel in Figure 15. We applied corrections of the form

$$
\log M_{\mathrm{BH}}^{\mathrm{Corr}}\left(\mathrm{C}_{\mathrm{IV}}\right)=\log M_{\mathrm{BH}}^{\mathrm{VP} 06}(\mathrm{C} \mathrm{IV})-b-a \log \frac{\lambda L_{\lambda}(1350 \AA)}{\lambda L_{\lambda}(5100 \AA)},
$$

where the coefficients $a$ and $b$ are listed in Table 7. For completeness, this table also shows the coefficients obtained when using the prescription A line widths of C IV, which are of similar magnitude and significance. Note that the uncertainties given for these coefficients have been determined after rescaling the errors such that the best fit has $\chi_{v}^{2} \equiv 1$.
The agreement between the rest-frame UV and optical BH mass estimates after applying this correction is remarkable, and the scatter of objects with group I and non-lower bound line widths has decreased from 0.30 to $0.11,0.23$ to $0.10,0.46$ to 0.25 , and 0.38 to 0.22 dex for panels (a)-(d) of Figures 7 and 16 , respectively. We find that the lowest scatter is between the $\mathrm{BH}$ masses estimated from the $\sigma_{l}$ of $\mathrm{C}$ IV $\sigma_{l}$ and the FWHM of either Balmer line. This supports our conclusion in the previous section that $\sigma_{l} \mathrm{C}$ IV $\mathrm{BH}$ masses have small random errors, even if their systematic errors may be much larger than those of the FWHM estimates due to blending of emission lines. Such a small scatter places strong constraints on the strength of a possible correlation between the mass residuals and any tertiary parameter. We find again that a slope different from unity is not required to describe the relation between the logarithm of the $\mathrm{C}$ IV and Balmer line $\mathrm{BH}$ masses.

Since BH mass estimates generally scale as $\Delta v^{2} L^{1 / 2}$ (Equations (4) and (6)), a naive interpretation of the reduced scatter is that we have simply shifted from showing $L_{1350 \AA}^{1 / 2}$ versus $\mathrm{L}_{5100 \AA}^{1 / 2}$ to $L_{5100 \AA}^{1 / 2}$ versus $L_{5100 \AA}^{1 / 2}$. The best-fit 
Table 7

Linear Fits to Correlations of BH Mass Residuals with AGN Color

\begin{tabular}{|c|c|c|c|c|c|}
\hline \multirow{2}{*}{$\begin{array}{l}\text { C IV } \\
\text { Width }\end{array}$} & \multirow{2}{*}{$\begin{array}{c}\text { Balmer } \\
\text { Line }\end{array}$} & \multicolumn{2}{|c|}{ Group I Estimates } & \multicolumn{2}{|c|}{ Group I and II Estimates } \\
\hline & & $\mathrm{a}$ & $\mathrm{b}$ & $\mathrm{a}$ & $\mathrm{b}$ \\
\hline \multicolumn{6}{|c|}{ Prescription A } \\
\hline \multirow[t]{2}{*}{$\sigma_{l}$} & $\mathrm{H} \beta$ & $0.64 \pm 0.13$ & $-0.13 \pm 0.06$ & $0.68 \pm 0.16$ & $-0.13 \pm 0.08$ \\
\hline & $\mathrm{H} \alpha$ & $0.58 \pm 0.15$ & $-0.11 \pm 0.06$ & $0.57 \pm 0.11$ & $-0.17 \pm 0.06$ \\
\hline \multirow[t]{2}{*}{ FWHM } & $\mathrm{H} \beta$ & $0.89 \pm 0.25$ & $-0.20 \pm 0.12$ & $0.95 \pm 0.22$ & $-0.19 \pm 0.11$ \\
\hline & $\mathrm{H} \alpha$ & $0.75 \pm 0.30$ & $-0.20 \pm 0.13$ & $0.79 \pm 0.15$ & $-0.23 \pm 0.08$ \\
\hline \multicolumn{6}{|c|}{ Prescription B } \\
\hline \multirow[t]{2}{*}{$\sigma_{l}$} & $\mathrm{H} \beta$ & $0.60 \pm 0.11$ & $-0.18 \pm 0.05$ & $0.68 \pm 0.17$ & $-0.16 \pm 0.08$ \\
\hline & $\mathrm{H} \alpha$ & $0.51 \pm 0.14$ & $-0.14 \pm 0.06$ & $0.58 \pm 0.16$ & $-0.22 \pm 0.08$ \\
\hline \multirow[t]{2}{*}{ FWHM } & $\mathrm{H} \beta$ & $0.86 \pm 0.25$ & $-0.23 \pm 0.12$ & $0.91 \pm 0.22$ & $-0.22 \pm 0.10$ \\
\hline & $\mathrm{H} \alpha$ & $0.72 \pm 0.30$ & $-0.23 \pm 0.13$ & $0.76 \pm 0.16$ & $-0.27 \pm 0.08$ \\
\hline \multicolumn{6}{|c|}{ Combined Sample } \\
\hline FWHM & $\mathrm{H} \beta$ & $0.82 \pm 0.18$ & $-0.40 \pm 0.07$ & $0.85 \pm 0.18$ & $-0.40 \pm 0.07$ \\
\hline
\end{tabular}

Notes. The fits discussed in Section 4.2 correspond those performed using the prescription B C IV line widths. Fits obtained using prescription A measurements are shown for completeness. The fits to the combined sample discussed in Section 5 are also reported here.

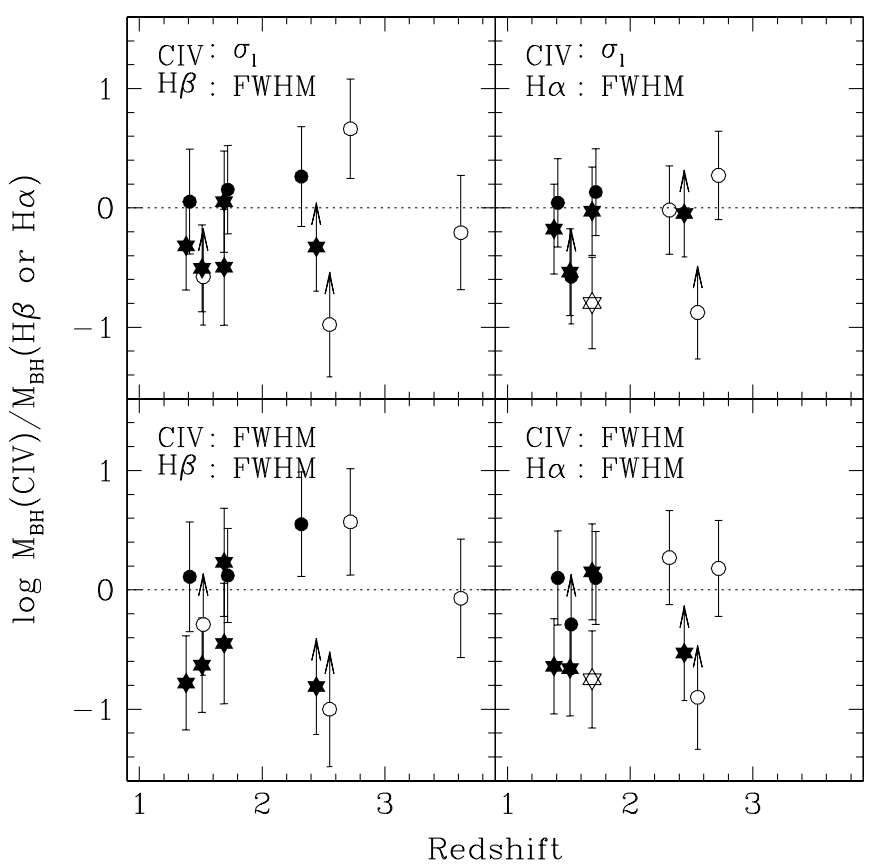

Figure 11. Same as Figure 8, but as a function of redshift.

correction is statistically different from simply replacing $L_{1350 \AA}$ by $L_{5100 \AA}$ by $1 \sigma-2 \sigma$, so it is not simply swapping the luminosities. More importantly, even if the slope was exactly $\alpha=1 / 2$, it reveals the crucial point that a significant fraction of any problems in reconciling $C I V$ and Balmer line estimates of $B H$ masses is due to the estimates of the continuum luminosities rather than any properties of either line.

There are three potential causes for a correlation of the mass ratio with the ratio of the continuum luminosities: (1) obscuration, (2) host contamination, and (3) non-universal AGN SEDs. Unfortunately, our analysis does not allow us to determine which $\mathrm{BH}$ mass estimate is more accurate. Extinction will reduce the rest-frame UV continuum luminosity while having little effect on the rest-frame optical luminosity. Conversely, host contamination will raise the optical luminosity while leaving the UV unchanged, as galaxies are typically brighter in the optical than

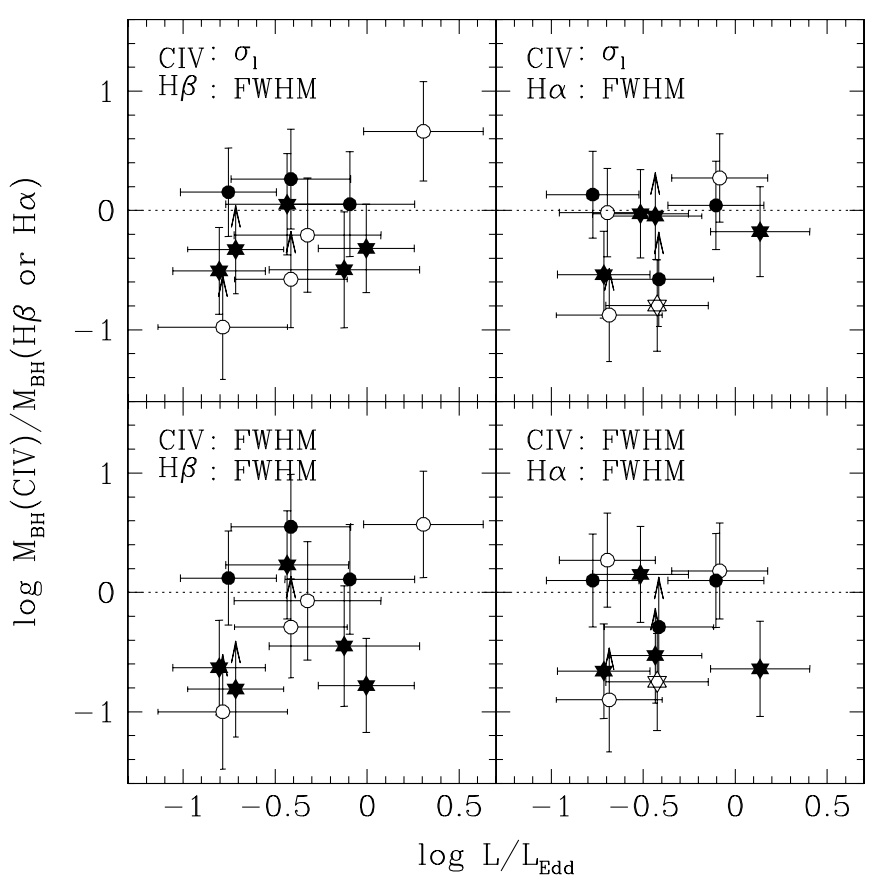

Figure 12. Same as Figure 8 , but as a function of the estimated Eddington ratio. We used a factor of 11.91 to convert between $\lambda L_{\lambda}(5100 \AA)$ and $L_{\mathrm{Bol}}$, as determined from the AGN SED of Assef et al. (2010).

in the UV. With respect to case (3), the radius of the BLR is really determined by the flux of the ionizing continuum $(\lambda<912 \AA)$. The $R_{\mathrm{BLR}}-L$ relations used to construct Equations (4), (5), and (6) implicitly assume a universal SED for all quasars, as they imply that the ionizing continuum can be uniquely predicted from the continuum luminosity at longer wavelengths. This approximation is likely to be better for the rest-frame UV continuum than for the optical. All three cases discussed would produce a slope of $a \simeq 0.5$ in Equation (8), simply representing the luminosity power indices in Equations (4), (5), and (6). This is generally shallower than the observed slope but within $2 \sigma$ of the best-fit relations. A larger sample is needed to fully determine if the slope of this correlation is statistically different from $\alpha \simeq 0.5$. We note that in order to create a slope larger than 


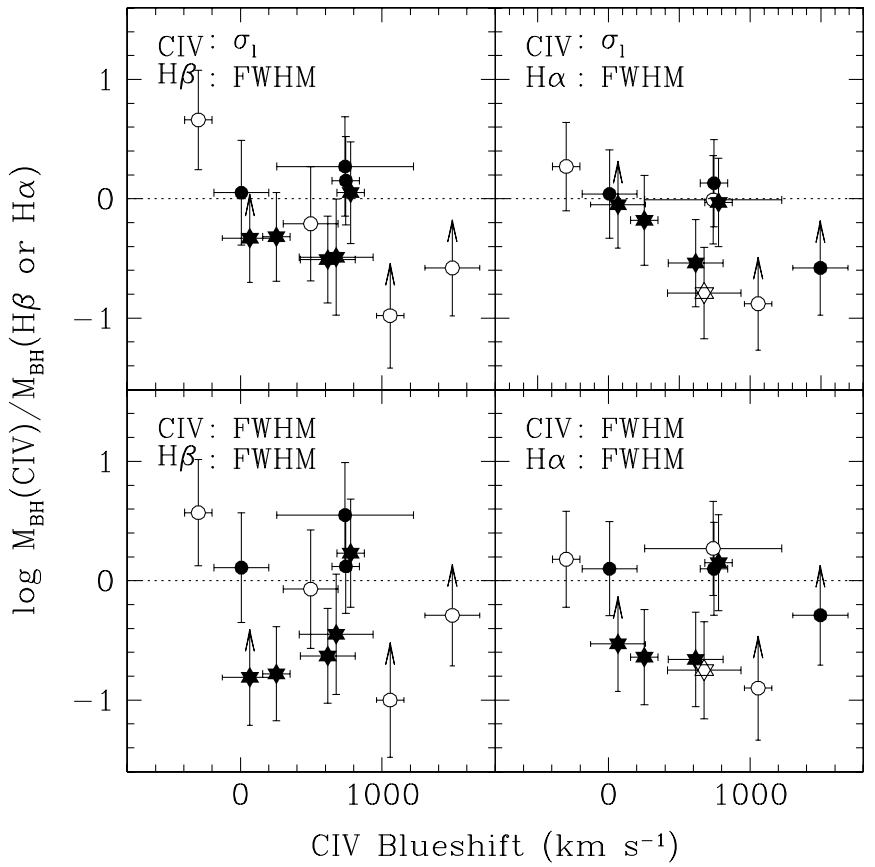

Figure 13. Same as Figure 8, but as a function of the blueshift of the $\mathrm{C}$ IV line.

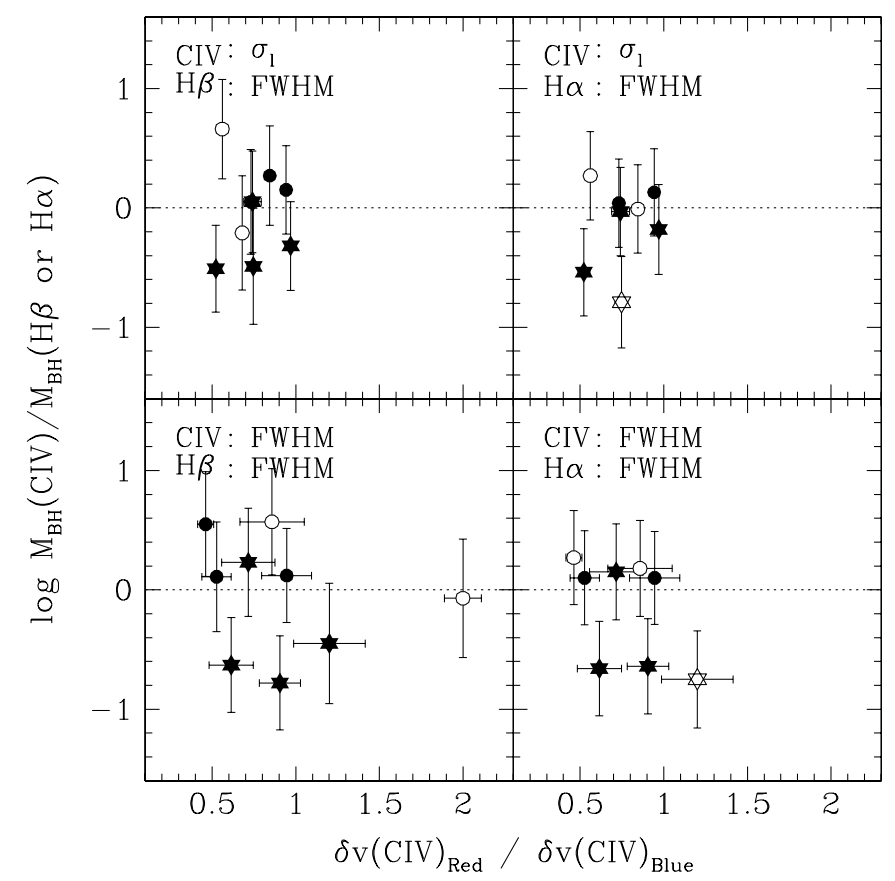

Figure 14. Same as Figure 8, but as a function of the asymmetry of the CIV line, parameterized as the ratio of the widths red and blue of the line centroid. We do not show lower bounds on the CIV line width as those objects do not have a well defined blue side width due to the presence of absorption.

0.5 , it would be necessary for the velocity widths of the quasar broad lines to be dependent on the ratio of the continuum luminosities. There is some evidence that the inclination angle of the accretion disk with respect to the line of sight may correlate with both the SED of the continuum (Gallagher et al. 2005 and references therein) and the FWHM of the broad $\mathrm{H} \beta$ line (Wills \& Browne 1986; Wills \& Brotherton 1995; Jarvis \& McLure 2006), although no such correlation is observed for the FWHM of C IV (Vestergaard et al. 2000, but see Decarli et al. 2008). Accretion disk inclination corrections, however, would act in the

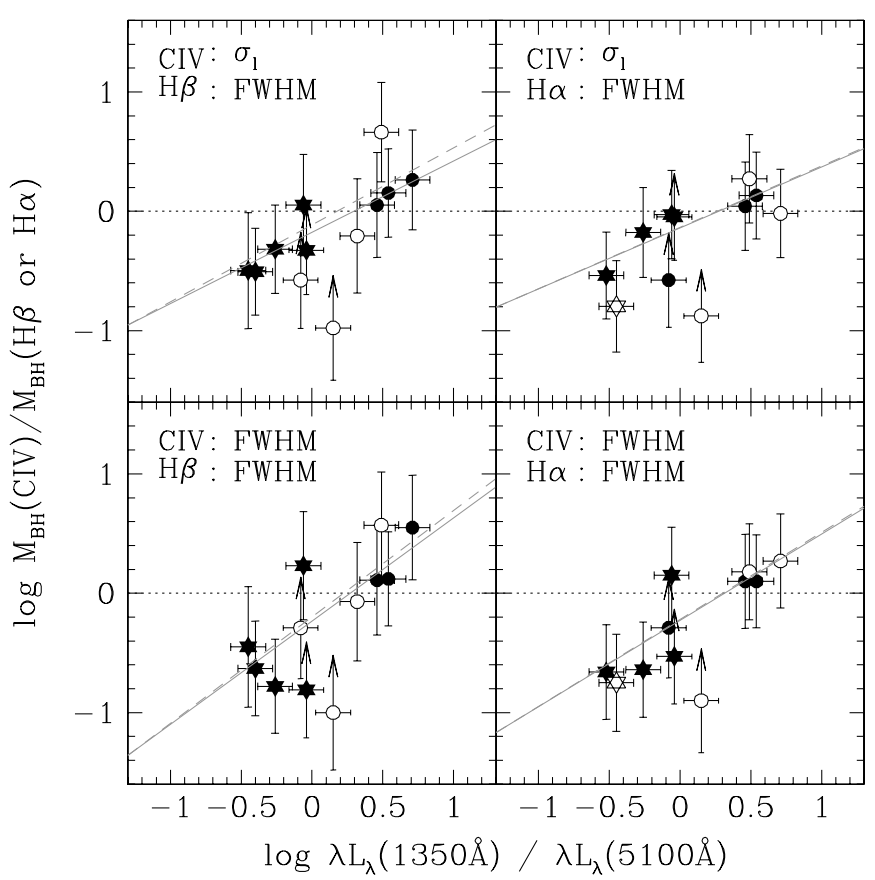

Figure 15. Same as Figure 8, but as a function of the ratio of the UV to optical continuum luminosities. The solid line shows the best-fit linear relation to all objects with group I Balmer line width estimates (solid symbols) and the dashed line shows the linear relation obtained when also including object with group II estimates (open symbols). Note that we do not include objects with lower bound $\mathrm{C}$ IV line widths on the fits.

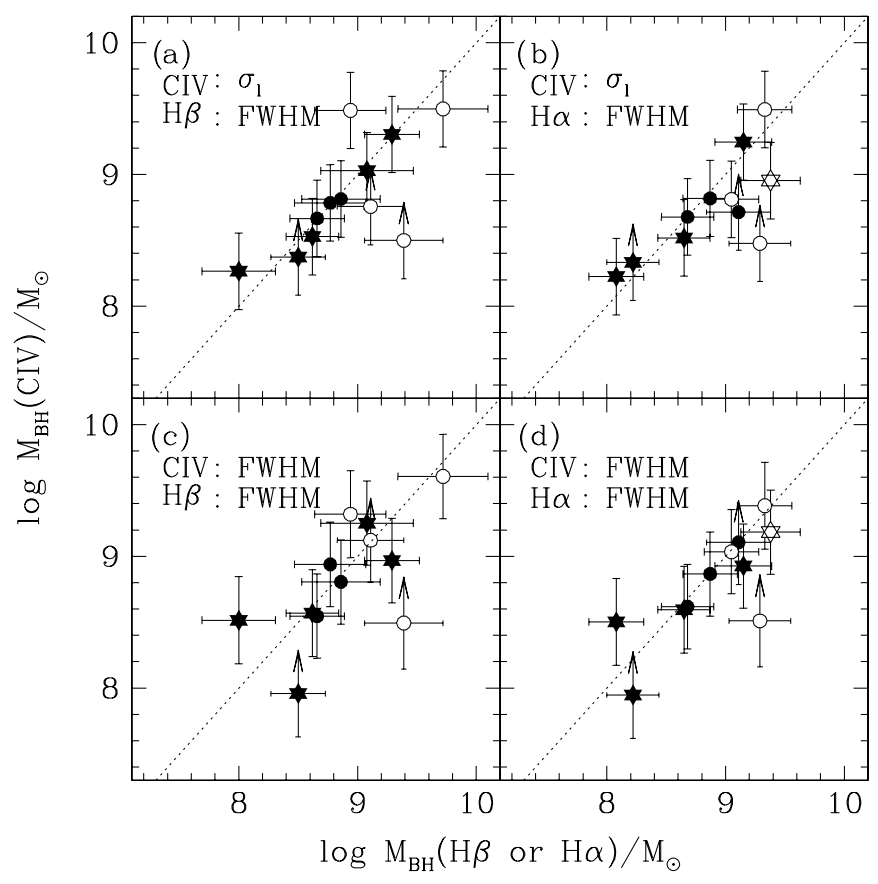

Figure 16. Same as Figure 7, but after correcting the $\mathrm{C}_{\mathrm{IV}} \mathrm{BH}$ masses for the dependence on the ratio of the UV to optical continuum luminosities observed in Figure 15.

opposite sense to the observed correlation and hence cannot be responsible for a slope in excess of 0.5 - disks with higher inclination angles (closer to edge-on) would appear to have higher FWHM of $\mathrm{H} \beta$ and bluer continua for a fixed "true" BH mass (i.e., not estimated from spectral features; see Gallagher et al. 2005 and references therein). 


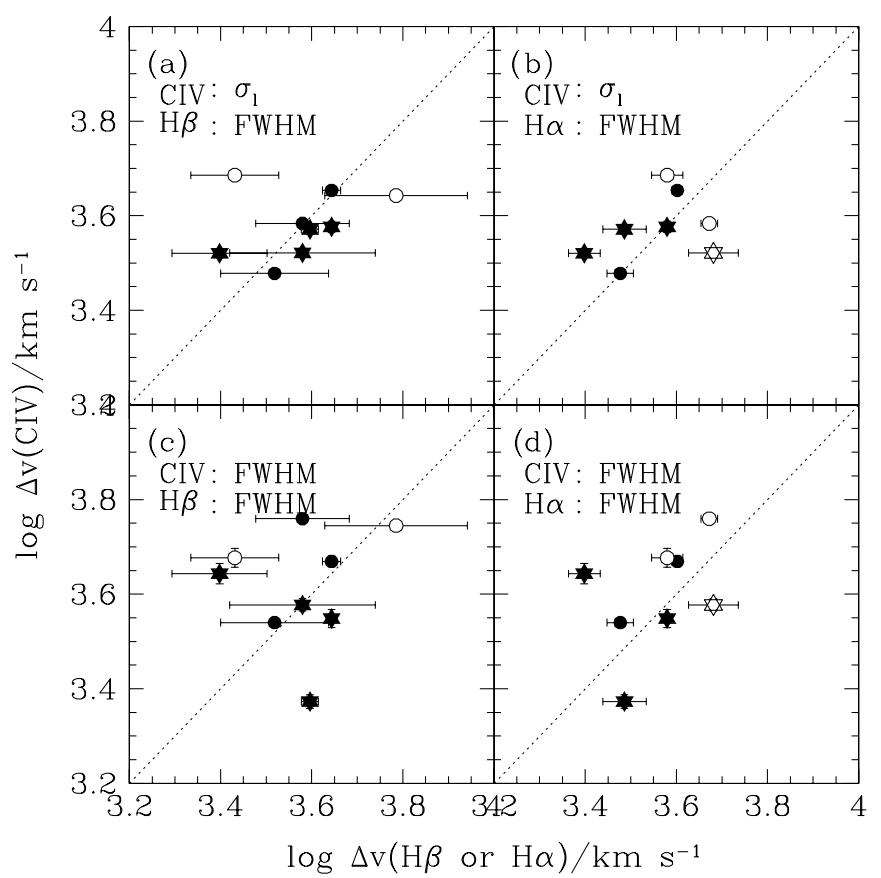

Figure 17. Comparison between the $\mathrm{C}$ IV and Balmer lines measured line widths. Points and lines have the same meaning as in Figure 7. We do not show lower bounds for clarity.

Our sample is likely representative of observations of the general quasar population in terms of reddening and host contamination. It could, in principle, have a larger typical reddening due to additional obscuration by dust associated with the lens, but this is unlikely to be important for our sample. Reddening by the lens galaxy will typically vary between quasar images. Falco et al. (1999) studied most of the objects in our sample and found that only two of them showed significant differential reddening: SBS0909+523 $(\Delta E(B-V)=0.2 \mathrm{mag}$ for image $\mathrm{B}$ with respect to $\mathrm{A}$; see also the Appendix) and $\mathrm{Q} 2237+0305(\Delta E(B-V)=0.18$ and $0.17 \mathrm{mag}$ for images $C$ and $D$ with respect to $A)$. Small but non-zero differential reddening was also detected for three other lenses (HE1104-1805, H1413+117, and B1422+231). The lensed quasars SDSS0246-0825, HS0810+2554, FBQ1633+3134, and SDSS1138+0314 were not part of the sample studied by Falco et al. (1999). We studied the latter object in Section 2.1 and concluded that images $\mathrm{B}$ and $\mathrm{C}$ did not show evidence for differential reddening between them, but there is no information in this regard for the other three quasars. Lensing can also alter host contamination in the quasar observations as compared to an unlensed case. The exact amount of host contamination depends on the size of the PSF and aperture used, the morphology of the lens, and the surface brightness profile of the quasar's host galaxy (see, e.g., Kochanek et al. 2001; Ross et al. 2009); however the zeroth-order effect is to not alter the amount of host contamination compared to an unlensed quasar.

While we have shown that the dominant source of scatter in the comparison between the $\mathrm{BH}$ mass estimates based on $\mathrm{C}$ IV and the Balmer lines is due to the continuum luminosities, we still wish to assess the relation between the widths of the different emission lines used. Figure 17 shows the comparison between the C IV and Balmer line widths. Note that we do not show measurements for which we only have lower bounds on the $\mathrm{C}$ IV width due to absorption. The best agreement is between $\sigma_{l}$ of $\mathrm{C}_{\mathrm{IV}}$ and FWHM of $\mathrm{H} \beta$, which is expected given that

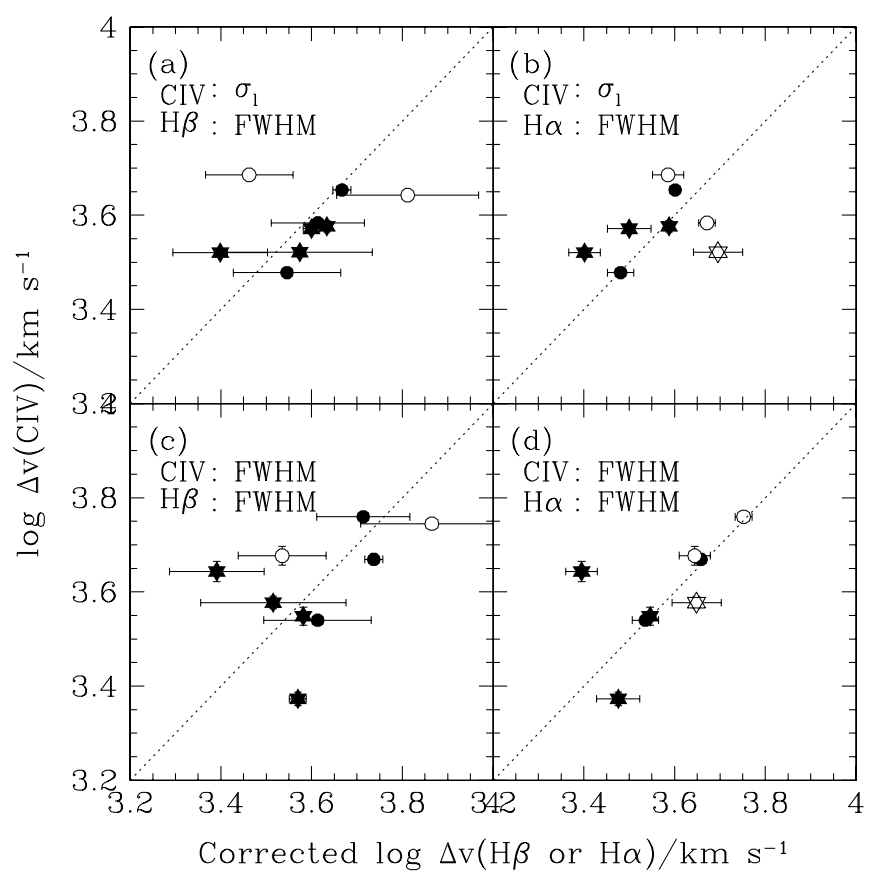

Figure 18. Comparison between the $\mathrm{C}$ IV and Balmer lines measured line widths after applying correction from Equation (9). Points and lines have the same meaning as in Figure 7 . We do not show lower bounds for clarity.

these measurements also give the lowest scatter in the BH mass estimates; however, a generally good agreement is also observed in all panels. We remind the reader, however, that the corrections we found between the $\mathrm{BH}$ mass estimates residuals and the ratio of the continuum luminosities did not have a slope of 0.5 . This implies that the ratio of the line widths may have a dependence on the luminosity ratio, with a power given by the excess of the slope from 0.5 . This could be a source of additional scatter in Figure 17, and so, instead of comparing the line widths directly, we also compare them after applying a correction based on the continuum luminosity estimates of the form

$$
\begin{gathered}
\log \Delta v(\mathrm{H} \beta \text { or } \mathrm{H} \alpha)_{\operatorname{corr}}=\log \Delta v(\mathrm{H} \beta \text { or } \mathrm{H} \alpha)+\frac{(a-0.53)}{2} \\
\times \log \frac{\lambda L_{\lambda}(1350 \AA)}{\lambda L_{\lambda}(5100 \AA)}+5 \times 10^{-3} \log \frac{\lambda L_{\lambda}(5100 \AA)}{10^{44} \mathrm{erg} \mathrm{s}^{-1}}
\end{gathered}
$$

as shown in Figure 18. Note that since we do not know the origin of the corrections, applying it to $\mathrm{H} \beta$ rather than $\mathrm{C}$ IV is a completely arbitrary decision made for illustration purposes. The agreement is now better and a correlation between the measurements is clear, suggesting that the widths of both lines are equally good tracers of $\mathrm{BH}$ mass. We have quantified the correlation between the different line widths in Table 8 using the Spearman rank-order coefficient, as we did for the $\mathrm{BH}$ mass residuals. After applying the correction from Equation (9), we find positive correlations which are typically statistically significant between the $\mathrm{C}$ IV and Balmer line widths. A weak anti-correlation, however, is measured between the FWHMs of $\mathrm{C}$ IV and $\mathrm{H} \beta$, but it is not statically significant.

\section{COMPARISON WITH OTHER STUDIES}

In Section 4.2, we used a sample of lensed quasars to compare $\mathrm{BH}$ masses based on observations of the $\mathrm{C}$ IV emission line and of the Balmer lines $\mathrm{H} \alpha$ and $\mathrm{H} \beta$. We found that the agreement between the rest-frame UV and rest-frame optical 
Table 8

Correlations of Line-width Estimates

\begin{tabular}{|c|c|c|c|c|c|c|c|c|}
\hline \multirow[t]{2}{*}{ C IV Prescription } & \multirow{2}{*}{$\begin{array}{c}\text { C IV } \\
\text { Width }\end{array}$} & \multirow{2}{*}{$\begin{array}{c}\text { Balmer } \\
\text { Line }\end{array}$} & \multicolumn{3}{|c|}{ Group I Estimates } & \multicolumn{3}{|c|}{ Group I and II Estimates } \\
\hline & & & $r_{s}$ & $N$ & $P_{\text {ran }}$ & $r_{s}$ & $N$ & $P_{\text {ran }}$ \\
\hline \multicolumn{9}{|l|}{ Without color correction } \\
\hline \multirow[t]{4}{*}{ Prescription A } & $\sigma_{l}$ & $\mathrm{H} \beta$ & 0.214 & 7 & 0.644 & 0.300 & 9 & 0.433 \\
\hline & & $\mathrm{H} \alpha$ & 0.700 & 5 & 0.188 & 0.786 & 8 & 0.021 \\
\hline & FWHM & $\mathrm{H} \beta$ & -0.143 & 7 & 0.760 & 0.000 & 9 & 1.000 \\
\hline & & $\mathrm{H} \alpha$ & 0.300 & 5 & 0.624 & 0.500 & 8 & 0.207 \\
\hline \multirow[t]{4}{*}{ Prescription B } & $\sigma_{l}$ & $\mathrm{H} \beta$ & 0.679 & 7 & 0.094 & 0.333 & 9 & 0.381 \\
\hline & & $\mathrm{H} \alpha$ & 0.900 & 5 & 0.037 & 0.524 & 8 & 0.183 \\
\hline & FWHM & $\mathrm{H} \beta$ & -0.143 & 7 & 0.760 & 0.000 & 9 & 1.000 \\
\hline & & $\mathrm{H} \alpha$ & 0.300 & 5 & 0.624 & 0.500 & 8 & 0.207 \\
\hline Combined Sample & FWHM & $\mathrm{H} \beta$ & 0.346 & 52 & 0.012 & 0.342 & 54 & 0.011 \\
\hline Combined Sample $(\mathrm{S} / \mathrm{N}>10)$ & FWHM & $\mathrm{H} \beta$ & 0.551 & 31 & $1.3 \times 10^{-3}$ & 0.539 & 33 & $1.2 \times 10^{-3}$ \\
\hline \multicolumn{9}{|l|}{ With color correction } \\
\hline \multirow[t]{4}{*}{ Prescription A } & $\sigma_{l}$ & $\mathrm{H} \beta$ & 0.571 & 7 & 0.180 & 0.550 & 9 & 0.125 \\
\hline & & $\mathrm{H} \alpha$ & 0.900 & 5 & 0.037 & 0.905 & 8 & 0.002 \\
\hline & FWHM & $\mathrm{H} \beta$ & 0.357 & 7 & 0.432 & 0.383 & 9 & 0.308 \\
\hline & & $\mathrm{H} \alpha$ & 0.400 & 5 & 0.505 & 0.738 & 8 & 0.037 \\
\hline \multirow[t]{4}{*}{ Prescription B } & $\sigma_{l}$ & $\mathrm{H} \beta$ & 0.929 & 7 & 0.003 & 0.467 & 9 & 0.205 \\
\hline & & $\mathrm{H} \alpha$ & 0.900 & 5 & 0.037 & 0.452 & 8 & 0.260 \\
\hline & FWHM & $\mathrm{H} \beta$ & 0.357 & 7 & 0.432 & 0.383 & 9 & 0.308 \\
\hline & & $\mathrm{H} \alpha$ & 0.400 & 5 & 0.505 & 0.667 & 8 & 0.071 \\
\hline Combined Sample & FWHM & $\mathrm{H} \beta$ & 0.326 & 52 & 0.018 & 0.323 & 54 & 0.017 \\
\hline Combined Sample $(\mathrm{S} / \mathrm{N}>10)$ & FWHM & $\mathrm{H} \beta$ & 0.602 & 31 & $3.4 \times 10^{-4}$ & 0.587 & 33 & $3.3 \times 10^{-4}$ \\
\hline
\end{tabular}

Notes. The table shows the correlation strength of the C IV and Balmer line-width estimates for both C IV line-width measurement prescription, quantified by the Spearman rank order coefficient, $r_{s}$. Results are shown for group I and the combination of groups I and II measurements. In each case, $N$ indicates the number of lenses used to estimate the correlation strength and $P_{\text {ran }}$ indicates the probability of observing such a correlation by chance if the variables are uncorrelated.

based $\mathrm{BH}$ masses is reasonably good. We also found that this agreement is even better once we apply an empirically determined correction based on the ratio of the $1350 \AA$ and $5100 \AA$ continuum luminosities. There have been a number of previous studies that have explored the relative accuracy of $\mathrm{BH}$ masses based on $\mathrm{C}$ IV and $\mathrm{H} \beta$, and they have reached both similar and opposite conclusions.

The studies of VP06, which we have discussed previously, and Dietrich \& Hamann (2004) found that C IV derived BH masses are consistent with those obtained from the width of $\mathrm{H} \beta$, and hence constitute a valid replacement as a mass estimator. Shemmer et al. (2004), Netzer et al. (2007), Sulentic et al. (2007), and Dietrich et al. (2009), however, reached opposite conclusions. Shemmer et al. (2004) concluded that BH masses derived from $\mathrm{C}$ IV were poorly matched to those obtained from $\mathrm{H} \beta$ and could be systematically different. They showed that for a sample of narrow-line Seyfert 1 galaxies, $\mathrm{C}$ IV based $\mathrm{BH}$ masses are larger by an average factor of $\sim 3$ with respect to those obtained from $\mathrm{H} \beta$. Dietrich et al. (2009) also found a large disagreement between the two estimates of the $\mathrm{BH}$ mass, but they found that using $\mathrm{C}$ IV tends to underestimate the $\mathrm{BH}$ masses by a factor of $\sim 1.7$, although the significance of this result is limited by the small number of objects (nine) in their sample. While Sulentic et al. (2007) also found significant disagreement, they argue that the magnitude of the offset depends on the spectroscopic characteristics of the quasar. Netzer et al. (2007), on the other hand, found no significant offset between the mass estimates, but argued there was also no discernible correlation between them. It is likely that many of the differences between the results of these studies are due to the use of different $R_{\mathrm{BLR}}-L$ calibrations, different $f$ factors, different prescriptions for measuring line widths, limited mass ranges, and data quality.
Here we take their measurements, where possible, and make estimates of the BH masses using Equations (4), (5), and (6). We caution the reader, however, that we are not redoing the linewidth and continuum luminosity measurements in a consistent manner and that this may be a significant source of additional scatter. We used all 21, 15, and 9 sources from VP06, Netzer et al. (2007) and Dietrich et al. (2009), respectively, with $\mathrm{H} \beta$ and $\mathrm{C}$ IV line FWHM and continuum luminosity measurements. We could not use 10,29, and 1 sources from these studies that lack either or both line widths, or any of the sources from Dietrich \& Hamann (2004), which lack measurements of the $5100 \AA$ continuum luminosity. We also could not use the sources of Sulentic et al. (2007), as they only report narrow-component subtracted C IV widths, which are not compatible with the rest of the measurements we discuss. We note that the 29 sources we could not use from the study of Netzer et al. (2007) also belong to the sample of Shemmer et al. (2004), for which the C IV line widths and UV continuum luminosities are not reported.

The left panel of Figure 19 compares the $\mathrm{C}$ IV and $\mathrm{H} \beta$ $\mathrm{BH}$ masses derived for all these objects along with those in our sample. A clear correlation is observed for the complete ensemble of objects, albeit with a considerable scatter of 0.41 dex. The scatter is comparable with the 0.46 dex we find for our sample of $\mathrm{CIV}$ and $\mathrm{H} \beta$ FWHM-based $\mathrm{BH}$ masses (see Section 4.2). A Spearman rank-order coefficient analysis returns $r_{s}=0.79$ with a probability of $P_{\text {ran }}=2 \times 10^{-12}$ that both mass estimates are uncorrelated. A linear fit to the left panel of Figure 19 of the form

$$
\log \frac{M_{\mathrm{BH}}\left(\mathrm{C}_{\mathrm{IV}}\right)}{10^{8} M_{\odot}}=m \log \frac{M_{\mathrm{BH}}(\mathrm{H} \beta)}{10^{8} M_{\odot}}+n
$$




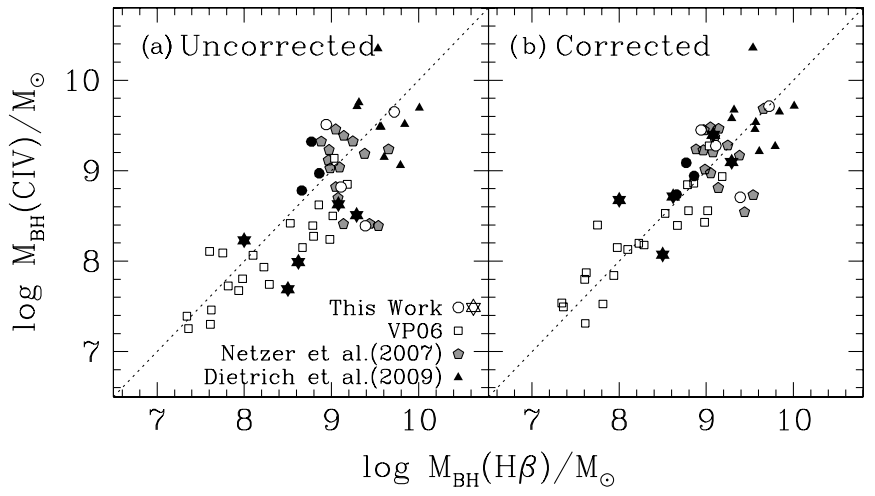

Figure 19. Panel (a) shows the BH masses estimated using Equations (4)-(6) for the objects in the samples of VP06 (open squares), Netzer et al. (2007; solid gray pentagons), and Dietrich et al. (2009; solid black triangles) for which this was possible (see Section 5 for details). Objects in our sample are shown by the solid and open six-pointed stars and circles, keeping the point style conventions used in previous plots. The dotted line shows where the masses are equal. Error bars are not shown in order to make the plot more legible. Panel (b) shows the results after applying the continuum slope correction from Table 7.

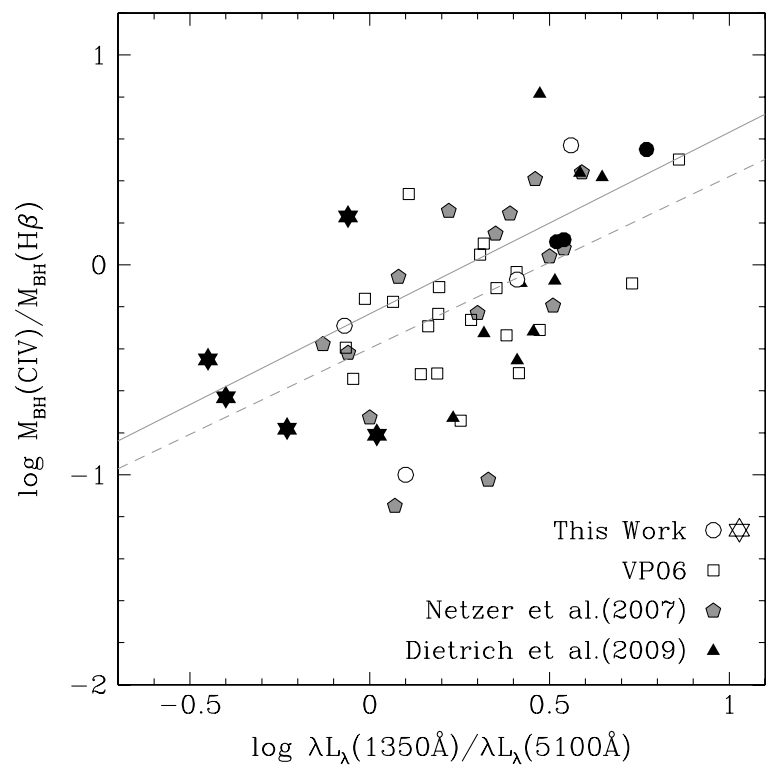

Figure 20. Residuals between $\mathrm{BH}$ masses estimated from the FWHM of the $\mathrm{C}$ IV and $\mathrm{H} \beta$ broad emission lines as a function of the logarithm of ratio of the continuum luminosities at $1350 \AA$ and $5100 \AA$ for the samples of VP06 (open squares), Netzer et al. (2007; solid gray pentagons), and Dietrich et al. (2009; solid black triangles) as well as our sample (solid and open six-pointed stars and circles). Point styles have the same definitions as in Figure 19. The solid line shows the best-fit linear relation for our data while the dashed line shows the best fit to the combined sample. Error bars are not shown in order to make the plot more legible.

returns a best-fit slope of $m=0.89 \pm 0.08$ and intercept of $n=-0.09 \pm 0.08$ (the measurement uncertainties were scaled to make $\chi_{v}^{2}=1$ before determining the uncertainties in the fit parameters). If we plot the residuals between the two BH mass estimates we find, just as in Section 4.2, that a significant correlation is observed with the ratio of the UV and continuum luminosities (Figure 20), but not with $\mathrm{BH}$ mass, redshift, Eddington ratio or the continuum luminosity at $5100 \AA$ (all shown in Figure 21), or with the continuum luminosity at $1350 \AA$ (not shown). The best-fit linear relation to the correlation between $\mathrm{BH}$ mass residuals and the ratio of the continuum luminosities, shown in Table 7, has a slope of $a=0.82 \pm 0.18$ and an intercept of $b=-0.40 \pm 0.07$. While the slope is consistent with the value obtained for our sample
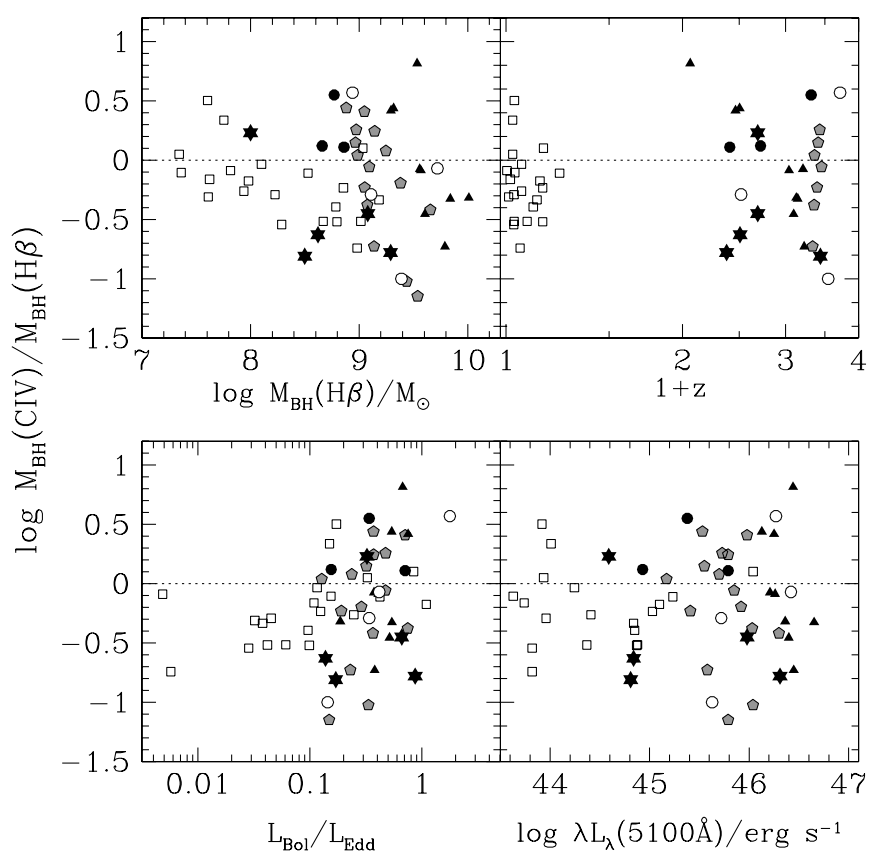

Figure 21. Residuals between BH masses estimated from the FWHM of the $\mathrm{C}$ IV and $\mathrm{H} \beta$ broad emission lines as a function of the logarithm of $\mathrm{H} \beta$-based $\mathrm{BH}$ mass (top left), redshift (top right), Eddington ratio (bottom left), and continuum luminosity at $5100 \AA$ (bottom right). Objects belong to the samples of VP06 (open squares), Netzer et al. (2007; solid gray pentagons), and Dietrich et al. (2009; solid black triangles) as well as our sample (solid and open six-pointed stars and circles). Point styles have the same definitions as in Figure 19. The dotted line shows where the masses are equal. Error bars are not shown in order to make the plot more legible.

alone $(a=0.86 \pm 0.25, b=-0.23 \pm 0.12)$, the intercept differs by approximately 0.2 dex (approximately $1.5 \sigma$ ). The offset is likely produced by the different prescriptions used to measure the width of the broad emission lines. Figure 19 also compares the $\mathrm{C}$ IV and $\mathrm{H} \beta$ derived masses after correcting for this correlation by applying Equation (8) (see Section 4.2 for details). While the strength of the correlation has not increased substantially $\left(r_{s}=0.80, P_{\text {ran }}=6.4 \times 10^{-13}\right)$, the scatter has decreased from 0.41 dex to 0.34 dex. This change is significantly more modest than that found for our sample of lensed quasars, but this is likely due to the inhomogeneous prescriptions used to measure the width of the emission lines. A linear fit of the form of Equation (10) to the relation between the $\mathrm{BH}$ mass estimates after applying the correction returns very similar parameters as before, with a best-fit slope of $m=0.88 \pm 0.07$ and intercept of $n=0.06 \pm 0.07$. Note that the measurement errors have again been scaled to make $\chi_{v}^{2}=1$ before estimating the uncertainties in the fit parameters. Given the inhomogeneity of the measurements used, the relatively small number statistics of the sample, their typically large error bars, and the likely intrinsic dispersion, however, we cannot currently determine whether the deviation from a slope of unity is significant or not.

As argued before, the inhomogeneity of the measurements can be a very significant source of scatter in the comparisons discussed above. We have shown in the previous section that with homogeneously analyzed, high $\mathrm{S} / \mathrm{N}$ spectra, the difference between the line widths is not the dominant source of scatter in the comparison between $\mathrm{C}$ IV and Balmer-line-based $\mathrm{BH}$ mass estimates. We would still like to assess whether the $\mathrm{C}$ IV and $\mathrm{H} \beta$ line widths are correlated in this combined sample. Figure 22 shows this comparison for all the objects used in this section with and without applying the correction based on Equation (9). 


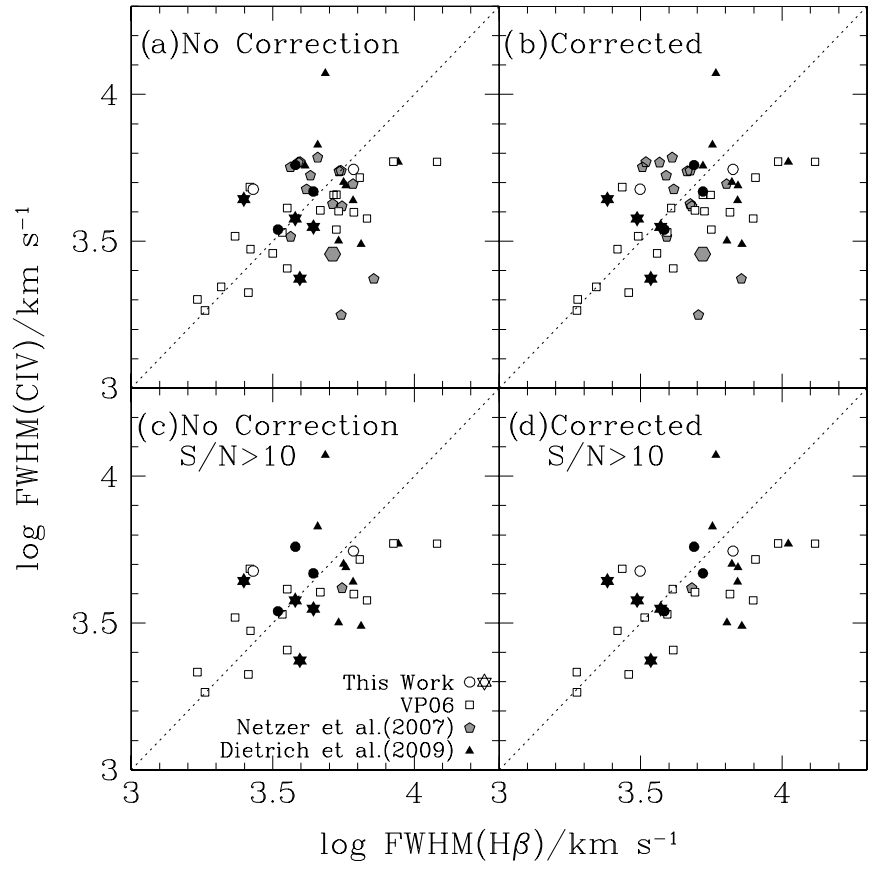

Figure 22. Comparison of the $\mathrm{C}$ IV and $\mathrm{H} \beta$ line widths for the sample we have compiled from the literature. Point types and line styles are the same as for Figure 19. The large gray hexagon shows SDSS1151+0340. Top panels show the complete literature sample while bottom panels only show objects with spectra that have continuum $S / N>10$ in the vicinity of $C$ IV.

While the scatter in Figure 22 is large, there is still a statistically significant (99\%) correlation between the measured line widths (see Table 8). Most of the scatter is due to the sample of Netzer et al. (2007). Upon inspection of the SDSS spectra used for that study, we find that almost all the outliers correspond to low $\mathrm{S} / \mathrm{N}$ spectra. Given that the C IV line is typically very complex, this can be a major source of uncertainty.

As an experiment, we obtained higher $\mathrm{S} / \mathrm{N}$ spectra for one of the outliers, SDSS1151+0340. It has the third most discrepant line-width ratio in the sense that the C IV line is too narrow compared to the Balmer lines. We obtained two independent spectra, one with OSMOS (Martini et al. 2011) at the MDM $2.4 \mathrm{~m}$ telescope and one with the Double Spectrograph (Oke \& Gunn 1982) at the Palomar 200 inch telescope. Due to poor weather conditions and aperture size, only the Double Spectrograph observations yielded a higher $\mathrm{S} / \mathrm{N}$ spectrum than that of SDSS. All three spectra of SDSS1151+0340 are shown in Figure 23. The spectrum obtained with the Double Spectrograph reveals that there is significant absorption near the $\mathrm{C}$ IV line, with two clear absorption troughs. These can be seen in the lower $\mathrm{S} / \mathrm{N}$ spectra, but are difficult to distinguish from the noise. Due to the very substantial absorption, it is not possible to reliably measure the width of the C IV line, and the width measurement of Netzer et al. (2007) should only be considered as a lower bound. While the SDSS spectrum of this source has the lowest continuum $\mathrm{S} / \mathrm{N}$ in their sample $(\mathrm{S} / \mathrm{N}=1.5)$, it is comparable to many of their other sources. The average continuum $\mathrm{S} / \mathrm{N}$ of the SDSS spectra is only 6.7, with all objects having a lower $\mathrm{S} / \mathrm{N}$ than any optical spectra in our lensed quasar sample. In particular, the second largest outlier in their sample also has the second lowest $\mathrm{S} / \mathrm{N}$ of 4.1 .

While our example comes from Netzer et al. (2007), low $\mathrm{S} / \mathrm{N}$ spectra are also present in all the additional samples we consider. If we eliminate objects with continuum $\mathrm{S} / \mathrm{N}<10$ in the vicinity of $\mathrm{C}_{\mathrm{IV}}$, the statistical correlation between the $\mathrm{H} \beta$

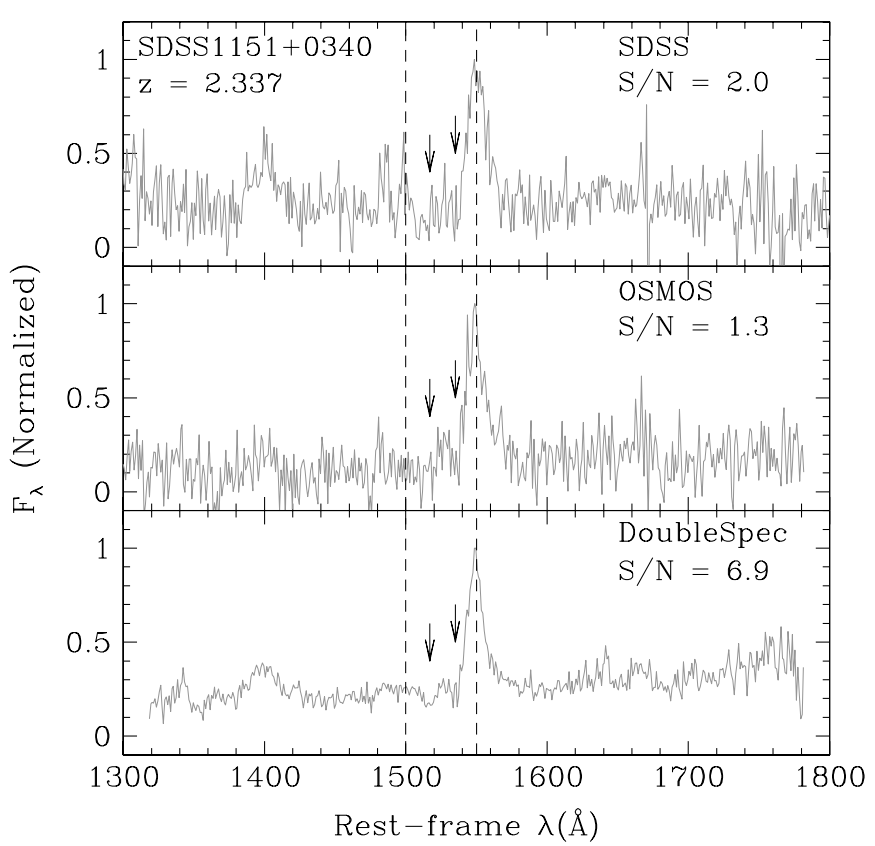

Figure 23. Spectra of the QSO SDSS1151+0340 obtained by SDSS (top), with MDM/OSMOS (middle) and with Palomar/Double Spectrograph(bottom). The arrows mark the probable absorption troughs near the $C$ IV line. The spectra have been resampled to a common resolution, and the continuum $\mathrm{S} / \mathrm{N}$ shown in the upper left corner of each panel has been calculated in the same way as for all other objects in our sample.

and C IV line widths increases dramatically. The bottom panels of Figure 22 show the comparison of the line widths in the absence of these objects, and a clear correlation is observed between the $\mathrm{C}$ IV and $\mathrm{H} \beta$ FWHM measurements, regardless of whether we apply the continuum luminosity based correction of Equation (9). These correlations are more statistically significant by about two orders of magnitude than when including the low $\mathrm{S} / \mathrm{N}$ spectra, with a probability of $\sim 5 \times 10^{-4}$ of not being real (see Table 8 for details). This suggests that the width of C IV is as good a tracer of $\mathrm{BH}$ mass as the width of $\mathrm{H} \beta$, with the caveat that high $\mathrm{S} / \mathrm{N}$ spectra of the rest-frame UV region are fundamental to accurately model the structure of the C IV emission line.

\section{CONCLUSIONS}

Using a sample of high-redshift gravitationally lensed quasars observed spectroscopically in the UV/optical and NIR, we have studied the agreement between SE BH mass estimators based on the $\mathrm{C}$ IV, $\mathrm{H} \beta$, and $\mathrm{H} \alpha$ broad emission lines. Our sample consists of 12 lensed quasars observed with HST by the CASTLES project. In particular, we have used the sample of NIR spectroscopic observations by GPL10 as a starting point and improved on it by (1) adding new NIR observations for three objects (SDSS1138+0314, SBS0909+253, and HS0810+2554), (2) adding high $\mathrm{S} / \mathrm{N}$, uniformly analyzed, optical spectroscopic observations for all targets, and (3) adding the missing restframe $\lambda L_{\lambda}(5100 \AA)$ luminosity estimates for SDSS0246-0852, HS0810+2554, and Q2237+030.

We described in detail all the methods we used to measure velocity widths and their uncertainties, rest-frame continuum luminosities, and to estimate the $\mathrm{BH}$ mass of each quasar using the $\mathrm{H} \beta, \mathrm{H} \alpha$, and $\mathrm{C}$ IV emission lines. We first compared the $\mathrm{C}$ IV $\mathrm{BH}$ mass estimates based on the FWHM and $\sigma_{l}$ line-width characterizations and the calibration of VP06 and found that, for our sample, the $\sigma_{l}$ based $\mathrm{BH}$ masses are systematically 
underestimated with respect to the FWHM-based ones by $0.13 \pm 0.06 \mathrm{dex}$ if using prescription $\mathrm{A}$ and $0.24 \pm 0.07 \mathrm{dex}$ if using prescription B. A similar offset is not observed in the VP06 data set. The difference probably arises from our treatment of the blending of the broad C IV emission line with the nearby broad HeII $\lambda 1640$ and Fe II emission redward of C IV, which is partly confirmed by the lower difference found for the prescription A measurements. This adds to the arguments in Denney et al. (2009) that $\sigma_{l}$ is not universally reliable for SE mass estimates in the presence of blending, as the results obtained are highly dependent on the exact prescription used for the line characterization. When comparing with $\mathrm{BH}$ masses derived from the $\mathrm{H} \alpha$ and $\mathrm{H} \beta$ broad emission lines, we find that $\mathrm{C}$ IV FWHM-based $\mathrm{BH}$ masses are not biased, reinforcing the conclusion that the bias is in the $\sigma_{l}$ estimates. We note, however, that the scatter between CIV FWHM and $\sigma_{l}$ derived masses is relatively small, suggesting that if a consistent prescription for measuring $\sigma_{l}$ is applied, $\sigma_{l}$ would be at least as accurate as FWHM. This is important because $\sigma_{l}$ measurements are significantly more reliable for complex line profile shapes and in the presence of narrow-line component residuals (Peterson et al. 2004; Denney et al. 2009).

We then compared the $\mathrm{C}$ IV and Balmer line $\mathrm{BH}$ mass estimates. After offsetting the $\mathrm{C}$ IV $\sigma_{l}$ masses to agree with the FWHM estimates, we find there is no significant offset between $\mathrm{C}$ IV and either Balmer line BH mass estimates. Averaged over the four possible $\mathrm{C}$ IV/Balmer line mass comparisons (see, for example, Figure 7), the offset is $-0.15 \pm 0.17 \mathrm{dex}$ and the scatter is 0.35 dex. Note that the error in the mean offset corresponds to the average of the errors of the four estimates, which is representative given that the estimates are not truly independent. The scatter of 0.35 dex is very close to the scatter of 0.34 dex found by Shen et al. (2008) between Mg II and $\mathrm{C}$ IV FWHM-based BH mass estimates, and significantly larger than the scatter of 0.22 dex they found between $\mathrm{Mg}$ II and $\mathrm{H} \beta$ FWHM-based BH masses.

We find that the residuals between the $\mathrm{CIV}$ and $\mathrm{H} \beta$ and $\mathrm{H} \alpha$ based mass estimates are not strongly correlated with the UV or optical continuum luminosities, redshift, or Eddington ratio, but we find a strong dependence on the ratio of the UV to optical continuum luminosities. If we correct for this color dependence, the agreement between the C IV and Balmer line estimates is remarkably good, with an average scatter of 0.18 dex, almost a factor of two smaller. We find the scatter is smallest-approximately $0.1 \mathrm{dex}$-when using the $\mathrm{H} \beta$ line and the $\sigma_{l}$ characterization of C IV rather than its FWHM. This observed correlation could be caused by (1) reddening, (2) host contamination, or (3) an object-dependent SED shape. The slope we observe is somewhat steeper than that expected in any of these cases, and may suggest a luminosity component to the line-width characterization of the broad emission lines. A larger sample is needed to accurately determine the slope of this correlation and determine its nature with certainty. More generally, the comparison shows that many of the problems in comparing $\mathrm{CIV}$ and Balmer line $\mathrm{BH}$ mass estimates are associated with the continuum luminosities rather than any potential physical complexities with the $\mathrm{C}$ IV lines. When we compare the line widths directly instead of the $\mathrm{BH}$ masses, we find that the width of C IV is well correlated with those of the Balmer lines once the correction based on the ratio of the continuum luminosities is applied.

Our conclusions are unchanged if we add 45 additional, but heterogeneously analyzed, $\mathrm{C}$ IV and $\mathrm{H} \beta$ estimates from VP06,
Netzer et al. (2007), and Dietrich et al. (2009). We used the published FWHM of both emission lines and rest-frame UV and optical continuum luminosities of these sources, but the mass calibrations used for our sample. There is a clear linear correlation between the $\mathrm{BH}$ mass estimates, and the residuals are again correlated with the ratio of the continuum luminosities. The residuals are not correlated with either continuum luminosity alone, redshift, $\mathrm{BH}$ mass, or Eddington ratio. We also find for this heterogeneous sample that the width of C IV is well correlated with that of $\mathrm{H} \beta$, particularly after we eliminate the objects with low $\mathrm{S} / \mathrm{N}$ C IV spectra. Relatively high $\mathrm{S} / \mathrm{N}$ spectra are essential to obtaining accurate line widths.

In summary, our results show that $\mathrm{C}$ IV is a good $\mathrm{BH}$ mass estimator but with small prescription-dependent offsets. The correlation of the mass residuals with the continuum slope could be a bias in either or both of the estimators. Determining the "blame" would require an independent mass estimate, but its existence should not be a surprise given that quasar SEDs are not universal (e.g., Yip et al. 2004). More generally, unless we are to believe that all properties of AGNs are determined by a single quantity, the BH mass, both SE mass estimates and RM radius estimates must depend on additional parameters. That the $\mathrm{BH}$ mass seems to dominate is convenient, but the excess scatter in mass and radius estimates beyond the measurement uncertainties requires either that the error estimates are incorrect or is evidence for additional parameters. One possibility is that radiation pressure plays a significant role (Marconi et al. 2008) and it could easily affect different lines in different ways. While there has been considerable recent effort to expand the range of BH masses included in these studies (e.g., Kaspi et al. 2007; Bentz et al. 2009; Botti et al. 2010), it is equally important to expand the range in other physical parameters such as spectral shape and Eddington ratio in order to better search for these additional correlations.

We thank Jenny E. Greene, Christopher Onken, Chien Y. Peng, Kristen Sellgren, Marianne Vestergaard, and Linda Watson for their help and suggestions that improved our work. We thank F. Courbin, E. Mediavilla, V. Motta, L. J. Goicoechea, S. Sluse, J. L. Tonry, L. Wisotzki, and J. Muñoz for sending us their optical spectra of Q2237+030, SDSS1138+0314, Q0957+561, HE1104-1805, B1422+231, and SBS0909+532. We thank F. Harrison for helping us obtain an optical spectrum of SDSS1151+0340. We also thank all the people in the LUCIFER science demonstration time team who did not participate directly in this work. We thank the anonymous referee for suggestions that helped improve our work. R.J.A. was supported in part by an appointment to the NASA Postdoctoral Program at the Jet Propulsion Laboratory, administered by Oak Ridge Associated Universities through a contract with NASA. C.S.K. is supported by NSF grants AST-0708082 and AST-1009756. B.M.P., M.D., and R.W.P. are supported by NSF grant AST-1008882. P.M. is supported by NSF grant AST-0705170. This research has made use of the NASA/IPAC Extragalactic Database (NED) which is operated by the Jet Propulsion Laboratory, California Institute of Technology, under contract with the National Aeronautics and Space Administration.

\section{APPENDIX \\ NOTES ON INDIVIDUAL OBJECTS}

In this section, we discuss some details of our linewidth and continuum measurements of individual objects. All 
$\mathrm{UV} /$ optical spectra, as well as the continuum and line-profile fits, are shown in Figure 4. LUCIFER spectra of SDSS1138+ 0314 and HS0810+2554 are shown in Figures 1 and 2, while the LIRIS spectra of SBS0909+532 are shown in Figure 3.

HS0810+2554. The CIV $\lambda 1549$ profile of HS0810 shows a small amount of absorption near the peak of the line. We interpolate over this region before making the line-width measurements and fitting the GH polynomial. Our results are consistent with or without the interpolation, as the absorption is weak and only seen near the very peak of the line. To fit the continuum and emission-line features that blended with the $\mathrm{H} \beta$ emission of HS0810+2554, a power-law continuum and Fe II broad-emission line template were fit to the spectrum based on the continuum regions listed in Table 4 and the rest-frame optical Fe II template of Boroson \& Green (1992) from observations of I Zw1 (see Wills et al. 1985; Dietrich et al. 2002, 2005, for more details). Narrow [O III] $\lambda \lambda 4959,5007$ emission was then removed by creating a template from a two-component Gaussian fit to the [O III $] \lambda 5007$ narrow line and then scaling it to [O III] $\lambda 4959$ based on standard emission line ratios. We could not remove narrow $\mathrm{H} \beta$ emission because such a component was not obvious in the spectrum. ${ }^{18}$ After subtracting these components, the remaining broad $\mathrm{H} \beta$ emission was fit with a $\mathrm{GH}$ polynomial, and the FWHM and line dispersion were measured from this fit as described in Section 3.1. The deblended spectrum of HS0810+2554, showing each component including the $\mathrm{GH}$ fit, is shown in Figure 2. Our $\mathrm{H} \beta$ FWHM measured from the LUCIFER spectrum of K. Mogren et al. (2011, in preparation) is consistent with that of GPL10.

SBS0909+532. We use the combined UV-optical-NIR spectrum of E. Mediavilla et al. (2011, in preparation) of images $\mathrm{A}$ and $\mathrm{B}$ of this object, based on a combination of HST STIS and WHT INTEGRAL and LIRIS observations. The UV section of the spectrum is shown in Figure 4 while the NIR section is shown in Figure 3. The C IV profile of SBS0909+532 showed a small absorption trough near observed frame $3600 \AA$ and we interpolated over this region before measuring widths. The SBS0909+532 C IV profile shape is "peaky" with broader wings at the base, and our GH fitting procedure was unable to satisfactorily fit this line profile, so we estimate errors based on the original spectra instead of a GH polynomial fit. For this object we only measure the UV continuum luminosity on image $\mathrm{A}$, as image $\mathrm{B}$ shows clear differential reddening with respect to A. We note that the Peng et al. (2006) mass quoted by GPL10 is based on Mg II, so using the C IV line-width measurements given in Table 3 provides the first estimate of a C IV-based $\mathrm{BH}$ mass for this object. For the IR spectra of E. Mediavilla et al. (2011, in preparation), shown in Figure 3, we removed narrowline components from the IR spectra using the [O III] $\lambda 5007$ line as a template and scaling it to the other narrow lines using standard emission-line ratios between lines of the same atomic species and basing the strength of the Balmer narrow lines on the ratio of $[\mathrm{O} \mathrm{III}] \lambda 5007 / \mathrm{H} \beta$ determined by inspection. We are not as confident in our narrow-line subtraction for this object as for the others because (1) we see residuals near the peak of $\mathrm{H} \beta$ and (2) the exact strength of $\mathrm{H} \alpha$ is uncertain because narrowline emission remains present after subtraction. The exact level of the residuals for $\mathrm{H} \alpha$ is unclear, since increasing the fraction of emission by as much as a factor of two does not result in an obviously improved subtraction. In the case of $\mathrm{H} \beta$, the residuals

\footnotetext{
18 GPL10 are similarly unable to isolate a narrow component in their
} observations of HSO810+2554. are not larger than expected based on the $\mathrm{S} / \mathrm{N}$ of the images, but for $\mathrm{H} \alpha$ we report uncertainties determined from the difference between the widths determined with or without the narrow-line subtraction. This results in an $\mathrm{H} \alpha$ FWHM uncertainty several times larger than would be estimated by our Monte Carlo simulations. Comparable $\sigma_{l}$ uncertainties are measured using both methods, because the line dispersion is far less dependent on the presence of a narrow-line component (see Denney et al. 2009). We measure the $\mathrm{H} \beta$ line widths from the $\mathrm{GH}$ fits to the profile and the $\mathrm{H} \alpha$ line widths directly from the data because the $\mathrm{GH}$ polynomials did not accurately fit the line profile. Image B may have a residual sky line peak just blueward of the $\mathrm{H} \beta$ narrowline component. The presence of this emission has little effect on our fits, however, since we measure consistent line widths if we interpolate under this emission to remove it. Our $\mathrm{H} \alpha$ and $\mathrm{H} \beta$ widths are consistent with those reported by GPL10.

Q0957+561. We use the HST STIS UV spectrum of both images obtained by Goicoechea et al. (2005). The rather strange $C_{\text {IV }} \lambda 1549$ line profiles in this object may indicate that there is absorption and/or that the profile shapes in individual images are affected by microlensing from the lens galaxy. However, since there was no definite source of uncertainty to correct for, we simply measured the observed line widths from each spectrum.

HE1104-1805. We use the EFOSC1 ESO $3.6 \mathrm{~m}$ telescope UV/optical spectrum of Wisotzki et al. (1995). The C IV $\lambda 1549$ profile shows a small amount of absorption near the peak of the line, similar to that of the HS0810+2554 C IV $\lambda 1549$ profile. We therefore apply the same treatment to this line as to the HS0810+2554 profile, and find similarly consistent results with or without interpolation.

$P G 1115+080$. Due to the severe absorption, both narrow and broad, in the CIV $\lambda 1549$ line profile in this object, we could not measure the C IV $\lambda 1549$ line width directly from the data. However, by masking out the absorption regions, we made a reasonable $\mathrm{GH}$ fit to this line profile, from which we measured the line widths given in Table 3 .

SDSS1138+0314. To measure the width of C IV and the UV continuum luminosity we use the FORS1 VLT spectra of images B and C obtained by Eigenbrod et al. (2006). The C IV $\lambda 1549$ line profile not only shows absorption in the blue side of the line, but is also particularly narrow and "peaky" with a broad base, similar to SBS0909+523. We were unable to reasonably approximate the profile shape with a sixth-order GH polynomial. However, since the $\mathrm{S} / \mathrm{N}$ of this spectrum was very high (see Table 2), we interpolated over the absorption with a secondorder polynomial, measured the line width directly from the interpolated data, and used this interpolated spectrum and the error spectrum formed with Equation (1) to derive uncertainties in the $C$ IV $\lambda 1549$ width measurement. Because of the combined effects of absorption and the narrow line profile (i.e., where the absorption could be masking the true width), we treat our $\mathrm{C}$ IV widths as lower limits. At rest-frame optical wavelengths, the difficulty in removing the blended narrow-line components of $\mathrm{H} \alpha$ and $\left[\mathrm{N}_{\mathrm{II}}\right] \lambda \lambda 6548,6583$ combined with our attempt to accurately fit the emission-line peak (often underestimated with line profile fits) led to an overestimate of the flux between the $\mathrm{H} \alpha$ and $\mathrm{N}_{\text {II }} \lambda 6583$ narrow lines. This overestimate does not significantly affect our width measurements. This object was not part of the GPL10 sample.

$H 1413+117$. This object is a BAL QSO and therefore a large portion of the C IV $\lambda 1549$ line profile is completely absorbed on the blue side. Hence, we adopt the C IV $\lambda 1549$ width measured 
from only the red side of the line, and we consider this to be a lower limit on the width.

$B 1422+231$. We use the LRIS Keck II UV/optical spectrum of Tonry (1998). We interpolated over the two small absorption troughs near $\sim 6875 \AA$ and $\sim 7020 \AA$ before measuring the C IV $\lambda 1549$ widths directly from the data. Our treatment of these regions did not affect the resulting $\mathrm{GH}$ fit to the data. From the GPL10 data, we cannot assess the reliability of their fit to the $\mathrm{H} \beta$ profile, because they plotted the $\mathrm{H} \beta$ spectrum of HE1104-1805 in place of the spectrum of B1422+231. In order to be conservative, we therefore flag B1422+231 as one of the objects with possible problems in the sample of GPL10.

$F B Q 1633+3134$. There is evidence for absorption in the blue side of C IV; however, it is not clear that a reliable interpolation could be made across this possible absorption. We measure the line width as is, and treat this measurement as a lower limit.

$Q 2237+030$. We use the FORS1 VLT UV/optical spectra of images C and D obtained by Eigenbrod et al. (2008). We follow the same prescription as for SDSS1138+0314 and Q0957+561 and use an average of the CIV line widths of each image to estimate $M_{\mathrm{BH}}$. This object shows C IV $\lambda 1549$ absorption in the red side of the line. We interpolate over this absorption with a third-order polynomial before measuring the line width and fitting the $\mathrm{GH}$ polynomial to the data. The interpolation creates a peak slightly higher than that observed in the original spectrum, but makes for a much more symmetric line profile, which is more typical of the core of $C$ IV $\lambda 1549$ line profiles, than either a linear or quadratic interpolation. This increase in the assumed line peak decreases our line-width measurements, but not significantly.

\section{REFERENCES}

Abazajian, K. N., Adelman-McCarthy, J. K., Agüeros, M. A., et al. 2009, ApJS, 182,543

Allington-Smith, J., Breare, M., Ellis, R., et al. 1994, PASP, 106, 983 Ageorges, N., Seifert, W., Juette, M., et al. 2010, Proc. SPIE, 7735, 53 Assef, R. J., Kochanek, C. S., Brodwin, M., et al. 2010, ApJ, 713, 970 Bentz, M. C., Peterson, B. M., Pogge, R. W., Vestergaard, M., \& Onken, C. A. 2006, ApJ, 644, 133

Bentz, M. C., Walsh, J. L., Barth, A. J., et al. 2009, ApJ, 705, 199

Blandford, R. D., \& McKee, C. F. 1982, ApJ, 255, 419

Boroson, T. A., \& Green, R. F. 1992, ApJS, 80, 109

Botti, I., Lira, P., Netzer, H., \& Kaspi, S. 2010, in IAU Symp. 267, Co-Evolution of Central Black Holes and Galaxies, ed. B. Peterson, R. Somerville, \& T. Storchi-Bergmann (Cambridge: Cambridge Univ. Press), 198

Cappellari, M., Verolme, E. K., van der Marel, R. P., et al. 2002, ApJ, 578, 787

Collin, S., Kawaguchi, T., Peterson, B. M., \& Vestergaard, M. 2006, A\&A, 456, 75

Decarli, R., Labita, M., Treves, A., \& Falomo, R. 2008, MNRAS, 387, 1237

Denney, K. D., Peterson, B. M., Dietrich, M., Vestergaard, M., \& Bentz, M. C. 2009, ApJ, 692, 246

Dietrich, M., Appenzeller, I., Vestergaard, M., \& Wagner, S. J. 2002, ApJ, 564, 581

Dietrich, M., Crenshaw, D. M., \& Kraemer, S. B. 2005, ApJ, 623, 700

Dietrich, M., \& Hamann, F. 2004, ApJ, 611, 761

Dietrich, M., Mathur, S., Grupe, D., \& Komossa, S. 2009, ApJ, 696, 1998

Dressler, A., Hare, T., Bigelow, B. C., \& Osip, D. J. 2006, Proc. SPIE, 6269, 13

Eigenbrod, A., Courbin, F., Meylan, G., Vuissoz, C., \& Magain, P. 2006, A\&A, 451,759

Eigenbrod, A., Courbin, F., Sluse, D., Meylan, G., \& Agol, E. 2008, A\&A, 480, 647

Fadely, R., Keeton, C. R., Nakajima, R., \& Bernstein, G. M. 2010, ApJ, 711, 246

Falco, E. E., Impey, C. D., Kochanek, C. S., et al. 1999, ApJ, 523, 617

Falco, E. E., Kochanek, C. S., Lehár, J., et al. 2001, in ASP Conf. Ser. 237, Gravitational Lensing: Recent Progress and Future Goals, ed. T. G. Brainerd \& C. S. Kochanek (San Francisco, CA: ASP), 25

Ferrarese, L., \& Merritt, D. 2000, ApJ, 539, L9

Fine, S., Croom, S. M., Bland-Hawthorn, J., et al. 2010, MNRAS, 409, 591

Gallagher, S. C., Richards, G. T., Hall, P. B., et al. 2005, AJ, 129, 567
Gaskell, C. M. 1982, ApJ, 263, 79

Gebhardt, K., Bender, R., Bower, G., et al. 2000, ApJ, 539, L13

Goicoechea, L. J., Gil-Merino, R., \& Ullán, A. 2005, MNRAS, 360, L60

Graham, A. W. 2007, MNRAS, 379, 711

Graham, A. W., Onken, C. A., Athanassoula, E., \& Combes, F. 2011, MNRAS, 412, 2211

Granato, G. L., De Zotti, G., Silva, L., Bressan, A., \& Danese, L. 2004, ApJ, 600,580

Greene, J. E., \& Ho, L. C. 2005, ApJ, 630, 122

Greene, J. E., Peng, C. Y., \& Ludwig, R. R. 2010, ApJ, 709, 937 (GPL10)

Gültekin, K., Richstone, D. O., Gebhardt, K., et al. 2009, ApJ, 698, 198

Hopkins, P. F., Hernquist, L., Cox, T. J., \& Kereš, D. 2008, ApJS, 175, 356

Hopkins, P. F., Hernquist, L., Cox, T. J., et al. 2005, ApJ, 630, 705

Hopkins, P. F., Robertson, B., Krause, E., Hernquist, L., \& Cox, T. J. 2006, ApJ, 652,107

Jahnke, K., \& Maccio, A. 2011, ApJ, 734, 92

Jarvis, M. J., \& McLure, R. J. 2006, MNRAS, 369, 182

Jiang, G., \& Kochanek, C. S. 2007, ApJ, 671, 1568

Kaspi, S., Brandt, W. N., Maoz, D., et al. 2007, ApJ, 659, 997

Kaspi, S., Maoz, D., Netzer, H., et al. 2005, ApJ, 629, 61

Kaspi, S., Smith, P. S., Netzer, H., et al. 2000, ApJ, 533, 631

Keeton, C. R. 2001, ApJ, submitted (arXiv:astro-ph/0102340)

Kelly, B. C., Bechtold, J., \& Siemiginowska, A. 2009, ApJ, 698, 895

Kelson, D. D. 2003, PASP, 115, 688

Kochanek, C. S., Keeton, C. R., \& McLeod, B. A. 2001, ApJ, 547, 50

Kochanek, C. S., Schneider, P., \& Wambsganss, J. 2004, in Proc. 33rd Saas-Fee Advanced Course, Part 2 of Gravitational Lensing: Strong, Weak \& Micro, ed. G. Meylan, P. Jetzer, \& P. North (Berlin: Springer)

Koopmans, L. V. E., Bolton, A., Treu, T., et al. 2009, ApJ, 703, L51

Laor, A., Bahcall, J. N., Jannuzi, B. T., et al. 1994, ApJ, 420, 110

Lehár, J., Falco, E. E., Kochanek, C. S., et al. 2000, ApJ, 536, 584

Leighly, K. M., \& Moore, J. R. 2004, ApJ, 611, 107

MacLeod, C. L., Ivezić, Ž., Kochanek, C. S., et al. 2010, ApJ, 721, 1014

Marconi, A., Axon, D. J., Maiolino, R., et al. 2008, ApJ, 678, 693

Marconi, A., \& Hunt, L. K. 2003, ApJ, 589, L21

Martini, P., Stoll, R., Derwent, M. A., et al. 2011, PASP, 123, 187

Marziani, P., Sulentic, J. W., Dultzin-Hacyan, D., Calvani, M., \& Moles, M. 1996, ApJS, 104, 37

McGill, K. L., Woo, J.-H., Treu, T., \& Malkan, M. A. 2008, ApJ, 673, 703

Mediavilla, E., Muñoz, J. A., Kochanek, C. S., et al. 2011, ApJ, 730, 16

Merritt, D., \& Ferrarese, L. 2001, in ASP Conf. Ser. 249, The Central Kiloparsec of Starbursts and AGN: The La Palma Connection, ed. J. H. Knapen, J. E. Beckman, I. Shlosman, \& T. J. Mahoney (San Francisco, CA: ASP), 335

Morgan, C. W., Byard, P. L., DePoy, D. L., et al. 2005, AJ, 129, 2504

Morgan, C. W., Kochanek, C. S., Morgan, N. D., \& Falco, E. E. 2010, ApJ, 712, 1129

Netzer, H., Lira, P., Trakhtenbrot, B., Shemmer, O., \& Cury, I. 2007, ApJ, 671, 1256

Oke, J. B., \& Gunn, J. E. 1982, PASP, 94, 586

Onken, C. A., \& Kollmeier, J. A. 2008, ApJ, 689, L13

Peng, C. Y. 2007, ApJ, 671, 1098

Peng, C. Y. 2010, in IAU Symp. 267, Co-evolution of Central Black Holes and Galaxies, ed. B. Peterson, R. Somerville, \& T. Storchi-Bergmann (Cambridge: Cambridge Univ. Press), 161

Peng, C. Y., Impey, C. D., Rix, H.-W., et al. 2006, ApJ, 649, 616

Peterson, B. M. 1993, PASP, 105, 247

Peterson, B. M. 2010, in IAU Symp. 267, Co-Evolution of Central Black Holes and Galaxies, ed. B. Peterson, R. Somerville, \& T. Storchi-Bergmann (Cambridge: Cambridge Univ. Press), 151

Peterson, B. M., Ferrarese, L., Gilbert, K. M., et al. 2004, ApJ, 613, 682

Richards, G. T., Vanden Berk, D. E., Reichard, T. A., et al. 2002, AJ, 124, 1

Ross, N. R., Assef, R. J., Kochanek, C. S., Falco, E., \& Poindexter, S. D. 2009, ApJ, 702, 472

Rusin, D., Kochanek, C. S., \& Keeton, C. R. 2003, ApJ, 595, 29

Schlegel, D. J., Finkbeiner, D. P., \& Davis, M. 1998, ApJ, 500, 525

Seifert, W., Appenzeller, I., Baumeister, H., et al. 2003, Proc. SPIE, 4841, 962

Shankar, F., Weinberg, D. H., \& Miralda-Escudé, J. 2009, ApJ, 690, 20

Shemmer, O., Netzer, H., Maiolino, R., et al. 2004, ApJ, 614, 547

Shen, Y., Greene, J. E., Strauss, M. A., Richards, G. T., \& Schneider, D. P. 2008, ApJ, 680, 169

Somerville, R. S., Hopkins, P. F., Cox, T. J., Robertson, B. E., \& Hernquist, L. 2008, MNRAS, 391, 481

Sulentic, J. W., Bachev, R., Marziani, P., Negrete, C. A., \& Dultzin, D. 2007, ApJ, 666, 757

Tonry, J. L. 1998, AJ, 115, 1

Tremaine, S., Gebhardt, K., Bender, R., et al. 2002, ApJ, 574, 740 
Tytler, D., \& Fan, X.-M. 1992, ApJS, 79, 1

Vacca, W. D., Cushing, M. C., \& Rayner, J. T. 2003, PASP, 115, 389

Vanden Berk, D. E., Wilhite, B. C., Kron, R. G., et al. 2004, ApJ, 601, 692

van der Marel, R. P., \& Franx, M. 1993, ApJ, 407, 525

Vestergaard, M., \& Peterson, B. M. 2006, ApJ, 641, 689 (VP06)

Vestergaard, M., Wilkes, B. J., \& Barthel, P. D. 2000, ApJ, 538, L103

Weymann, R. J., Carswell, R. F., \& Smith, M. G. 1981, ARA\&A, 19, 41

Wilhite, B. C., Brunner, R. J., Grier, C. J., Schneider, D. P., \& vanden Berk, D. E. 2008, MNRAS, 383, 1232

Wills, B. J., \& Brotherton, M. S. 1995, ApJ, 448, L81
Wills, B. J., \& Browne, I. W. A. 1986, ApJ, 302, 56

Wills, B. J., Netzer, H., Brotherton, M. S., et al. 1993, ApJ, 410, 534

Wills, B. J., Netzer, H., \& Wills, D. 1985, ApJ, 288, 94

Wilkes, B. J. 1984, MNRAS, 207, 73

Wisotzki, L., Koehler, T., Ikonomou, M., \& Reimers, D. 1995, A\&A, 297, L59

Woo, J.-H., Treu, T., Malkan, M. A., Ferry, M. A., \& Misch, T. 2007, ApJ, 661, 60

Yip, C. W., Connolly, A. J., Vanden Berk, D. E., et al. 2004, AJ, 128, 2603

Zu, Y., Kochanek, C. S., \& Peterson, B. M. 2011, ApJ, 735, 80 